\title{
MOBILITY MANAGEMENT IN
}

\section{THE FUTURE MOBILE NETWORK}

Morteza Karimzadeh Motallebi Azar

3GPP 


\section{MOBILITY MANAGEMENT IN THE FUTURE MOBILE NETWORK}

Morteza Karimzadeh Motallebi Azar 


\section{Graduation committee:}

Chairman:

Supervisor:

Supervisor:

Members:

Prof. dr. T. Taleb

Prof. dr. ir. S.M. Heemstra de Groot

Prof. dr. ir. B.R.H.M. Haverkort

Prof. dr. ir. G.J. Heijenk
Prof. dr. J.N. Kok

Prof. dr. J.L. van den Berg

Prof. dr. ir. A. Pras

Aalto University

Eindhoven University of Technology

University of Twente

University of Twente

\section{Funding sources:}

EU FP7 Mobile Cloud Networking - 318109

IDS Ph.D. Thesis Series No. 18-462

Institute on Digital Society

P.O. Box 217, 7500 AE

Enschede, The Netherlands

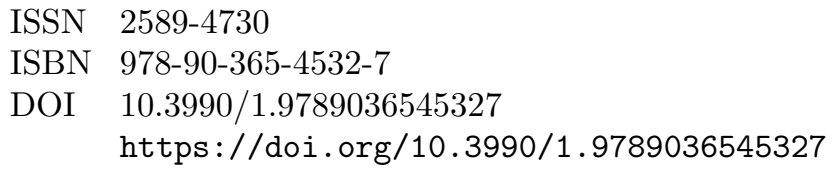

ISSN 2589-4730

ISBN $978-90-365-4532-7$

DOI $10.3990 / 1.9789036545327$

https://doi.org/10.3990/1.9789036545327

Type set with $\mathrm{LAT}_{\mathrm{E}} \mathrm{X}$.

Cover designed by: Optima, Rotterdam, The Netherlands.

Printed by: Optima, Rotterdam, The Netherlands.

Copyright @ 2018 Morteza Karimzadeh Motallebi Azar 


\title{
MOBILITY MANAGEMENT IN THE FUTURE MOBILE NETWORK
}

\author{
DISSERTATION
}

to obtain

the degree of doctor at the University of Twente, on the authority of the rector magnificus, Prof. dr. T.T.M. Palstra,

on account of the decision of the graduation committee, to be publicly defended

on Thursday the $19^{\text {th }}$ April 2018 at 16:45 hours

by

\section{Morteza Karimzadeh Motallebi Azar}

Born on the $21^{\text {th }}$ March 1975

in Urmia, Iran 
This dissertation has been approved by:

Prof. dr. J.L. van den Berg (Supervisor)

Prof. dr. ir. A. Pras (Supervisor) 


\section{Acknowledgments}

It was around 5 years ago, a sunny summer in Milan where I met Prof. Yevgeni Koucheryavy, who was there for vacation, enjoying sightseeing and talking about me looking for opportunities to pursue my Ph.D. studies and that was start of the journey! Sometime after leaving Milan, he contacted me to let me know about a vacancy in Design and Analysis of Communication Systems (DACS) research group at the University of Twente. I'd like to thank him for prompting start of such a valorous period in my life.

I would like to take this opportunity to express my earnest appreciation to all people who helped me one way or another during my Ph.D. study. Though I may forget to personally mention all and do sincerely apologize in advance.

My decent promoters Prof. Hans van den Berg and Prof. Aiko Pras who this thesis would not have been possible without their continuous guide and giving me the freedom to choose the direction for my research. The dignified chair of DACS, Prof. Boudewijn Haverkort with his persistent support of all the group members. My committee members, Prof. Tarik Taleb, Prof. Sonia Heemstra de Groot, Prof. Boudewijn Haverkort and Prof. Geert Heijenk for accepting to be in my committee and their valuable comments on my thesis.

My colleagues at DACS who I feel so lucky to share a group with. Very kind and competent people who made every single day a pleasant experience. In particular, my office mates Mattijs and Wouter. An special thank to Luuk who enjoyed translating the abstract of my Ph.D. thesis to Dutch. Jeanette who has been kind and caring like a mother and making things happen so fast and smooth. My colleagues from the Mobile Cloud Networking project who I learned a lot and shared very fun times during our project trips.

My paranymphs Elham Goumeh and Meisam Yousefzadeh for their support and help during the past month. My friends from home country who we shared many nice and memorable times together while far away from the families; Siavash and Mitra, Saeed and Zhaleh, Farhad and Elahe, Leila, Elham, Meysam and Hajar, Alireza and Barbara, Sina, Amir and Mina, Mohammad and Neda, Aidin and Maral, Mohammadreza and Anna, Mojtaba and Sadaf, Afshin and Elham, Shahin and Soodeh, Hassan, Davood, Alireza and Zahra, Niloofar, Mahroo, Hamed and Maryam and Morid. 
My parents in-law with their invaluable help and support. Thank you for raising an amazing daughter and helping her to become the strong woman she is today. My brother in-law Hamed, my sister in-law Mozhgan and their sweet kids, Anis and Tanin for bringing so much joy and light into my life.

My parents who have always been supportive during all stages of my life and gave me the freedom to follow my desires. My siblings, Mojtaba, Rahimeh, Marzieh, Mahdieh and Mostafa who I have shared a lifetime of bittersweet memories.

And my best friend, my love Mozhdeh, with whom we have successfully passed through all stages of our shared life hand-in-hand and strong. She has always been there for me with all her kind heart and making me feel so strong by always believing in me. 


\section{Abstract}

In the last years, with the worldwide deployment of mobile networks and the commercial success of smart portable devices e.g., smart-phones and tablets, the use of mobile data services and data traffic has exploded. This growth is foreseen to remain in future years, due to the continuous increase in popularity of mobile services and novel mobile applications, e.g., Internet of Things (IoT) and Machine to Machine (M2M). Mobile network operators and technology vendors are therefore strongly interested in the development of methods and strategies for enhancing the scalability of the overall system to cope with the future traffic growth.

The current mobile network architectures are heavily hierarchical and centralized, which implies that all traffic must be traversed through a centralized core entity. This makes the network prone to several limitations, e.g., suboptimal communication paths, low scalability, signaling overhead, and single point of failure. The hierarchy of the data forwarding procedure in current network architectures can be eliminated by letting IP flows be routed in a more flexible way. One of the key enablers for this is to shift towards flatter (decentralized) network architectures. This approach allows to place distributed anchor points in the proximity of the radio access network to locally handle Mobile Nodes' (MNs') connections and data traffic, and accordingly reduce the load of traffic in the core network.

Long-Term Evolution (LTE) is expected to be the leading mobile networking technology in the coming decade. It is estimated that LTE network will handle about $79 \%$ of the worldwide mobile data traffic by 2021. Therefore, our focus in this thesis is on LTE. We first review the current (centralized) LTE network system, and next propose and extensively investigate an approach to realize deployment of an LTE network with decentralized architecture.

Although, decentralization of the LTE network architecture would be, on the long term, a sustainable and cost-effective approach to deal with the future data traffic demands, its actual gain on the network performance and efficiency is an open question. Hence, we carry out a hybrid study comprising simulation and analytical models to compare the efficiency of the current and decentralized LTE network architectures. Particularly, our analysis for both network architectures presents numerical results, quantifying the impact of the number of attached 
MNs on the load at network routers and links, on the latency, and on the required processing in data and control planes.

When dealing with the transition from the centralized and hierarchical LTE network architecture to a decentralized one, mobility management is one of the key functions that needs to be adapted to the new architecture. In the current LTE system, the mobility management function follows a centralized scheme, in which the Packet Data Network Gateway (PGW) aggregates the traffic flows to and from the access networks, and redirects traffic flows to MNs' new locations, for those moving from one access network to another (i.e., from one Serving Gateway (SGW) to another). However, in an LTE network with decentralized architecture, the PGW and SGW functions are co-located into single entities (i.e., $\mathrm{S} / \mathrm{PGWs}$ ) closer to the radio access network, that handle the MNs' connection functions, data traffic and mobility locally. This, demands additional mechanisms to maintain the MN's ongoing data session active, when it performs a handover with $\mathrm{S} / \mathrm{PGW}$ relocation.

To address this, we develop, evaluate and compare three novel mechanisms. Our first solution exploits the Network Address Translation (NAT) technique, which is widely used in the current Internet. Therefore, its implementation is feasible with limited overhead and complexity.

In the second solution, we take the advantage of the Software Defined Network $(\mathrm{SDN})$ paradigm on detaching the control plane from data plane, to manage MNs' mobility in a decentralized LTE network. SDN is one of the core technologies in the transformation of current mobile networks to next generation networks (i.e., 5G). It is an essential enabler for many anticipated features and functionalities of $5 \mathrm{G}$ networks, such as elasticity, scalability, adaptability, slice-ability and program-ability. Knowing this, makes the proposed solution feasible and ideally suited for the next generation of mobile networks.

The NAT-based and SDN-based solutions conduct the MNs' mobility management at the network layer. This accordingly, hides any changes from the upper layers and keeps the end points unaware from the related functions. However, these approaches demand to introduce new components in the network and some infrastructural modifications. In the third solution, we benefit from the Multipath-TCP (MPTCP) protocol feature on supporting multiple IP addresses for a single data-stream and its break-before-make option to handle MNs' mobility. This solution keeps the network infrastructure intact and implements the whole mobility management functionality in the transport layer of only the end host entities. MPTCP is already deployed on smart-devices working with iOS (since version 7) or Android (since version 4.4) mobile operating systems. This makes the proposed approach readily feasible to implement at large scale with minimal overhead and complexity. Finally, we make a comparison of the proposed mobility management solutions and discuss their pros and cons. 


\section{Samenvatting}

De wereldwijde uitrol van mobiele netwerken, in combinatie met het commerciële succes van mobiele apparaten zoals smart-phones en tablets, heeft gezorgd voor een enorme stijging van zowel mobiel dataverkeer als het gebruik van mobiele datadiensten. Deze groei blijft in de toekomst aanhouden door de toenemende populariteit van deze diensten, alsmede de opkomst van nieuwe mobiele applicaties gerelateerd aan bijvoorbeeld Internet of Things (IoT) en Machineto-Machine (M2M) communicatie. Technologieleveranciers en beheerders van mobiele netwerken zijn daarom zeer geïnteresseerd in het ontwikkelen van methodes om de schaalbaarheid van hun netwerken en systemen te verbeteren en de verwachte verkeersgroei aan te kunnen.

De huidige mobiele netwerkarchitectuur is hiërarchisch en gecentraliseerd, met als gevolg dat al het verkeer door een centraal punt loopt. Dit heeft nadelige gevolgen, zoals suboptimale communicatiepaden, geringe schaalbaarheid, overhead in de communicatie, en een single point of failure. De hiërarchie in het doorsturen van data in huidige netwerkarchitecturen kan worden geëlimineerd door IP flows op een flexibelere manier te routeren. Hiervoor is een verschuiving naar plattere, gedecentraliseerde netwerkarchitecturen van belang. Bij zo'n aanpak worden gedistribueerde ankerpunten dicht bij het aansluit-/radionetwerk geplaatst om verkeer van de Mobile Nodes (MNs) zo veel mogelijk lokaal af te handelen, en zodoende de belasting van het centrale netwerk te verminderen.

Long-Term Evolution (LTE) zal de komende 10 jaar de dominante mobiele netwerktechnologie zijn. Naar verwachting wordt $79 \%$ van het wereldwijde mobiele dataverkeer in 2021 afgehandeld door LTE netwerken. In het licht hiervan focussen we in ons onderzoek op LTE. We bespreken eerst het huidige (gecentraliseerde) LTE systeem, en ontwikkelen en onderzoeken vervolgens een methode om een gedecentraliseerd LTE systeem te realiseren.

Ook al is het decentraliseren van de LTE netwerkarchitectuur een veelbelovende aanpak om de schaalbaarheid te vergroten, het blijft een open vraag in welke mate de prestatie en efficiëntie van het LTE netwerk hierdoor verbetert. In dit proefschrift worden beide netwerkarchitecturen in dit opzicht geanalyseerd. In het bijzonder kwantificeert deze analyse het effect van het aantal MNs verbonden met het netwerk op de belasting van de routers en de verbindingen 
in het netwerk, alsmede het effect op de vertragingen die worden opgelopen in het netwerk.

De overgang van de centrale en hiërarchische LTE netwerkarchitectuur naar een gedecentraliseerde architectuur vereist aanpassingen in het mobility management. Dat is het tweede hoofdonderwerp van het onderzoek in dit proefschrift. In het huidige LTE systeem wordt mobility management op een gecentraliseerde manier geregeld, waarbij de Packet Data Network Gateway (PGW) verkeer van en naar de aansluitnetwerken aggregeert en doorstuurt naar de nieuwe locaties van de MNs wanneer de bijbehorende gebruikers wisselen van aansluitnetwerk (bijvoorbeeld bij de overgang van de ene Serving Gateway (SGW) naar de andere). Echter, in een LTE netwerk met gedecentraliseerde architectuur zijn de functionaliteiten van de PGW en SGW dichter bij het aansluitnetwerk geplaatst (samengevoegd in één entiteit, de $\mathrm{S} / \mathrm{PGWs}$ ), waar het verkeer en de mobiliteit van $\mathrm{MNs}$ lokaal kan worden afgehandeld. Dit vereist i.h.b. nieuwe technieken om sessies van MNs actief te houden gedurende een handover waarbij van S/PGW gewisseld wordt.

Om dit te realiseren, ontwikkelen en vergelijken we drie nieuwe aanpakken. De eerste is gebaseerd op Network Address Translation (NAT), een techniek die veelvuldig gebruikt wordt in het huidige Internet. De implementatie is daarmee in principe te realiseren zonder veel overhead of complexiteit.

De tweede oplossing om de mobiliteit van MNs in een gedecentraliseerd LTE netwerk te regelen is gestoeld op het Software Defined Network (SDN) paradigma. SDN is één van de core technologiën voor de overgang van huidige mobiele netwerken naar die van de toekomst, zoals 5G netwerken. Het zorgt voor essentiële en langverwachte mogelijkheden, met name als het gaat om het realiseren van meer flexibiliteit en adaptiviteit van de netwerken. Dit maakt onze tweede oplossing realiseerbaar en zeer geschikt voor de volgende generatie van mobiele netwerken.

In deze op NAT en SDN gebaseerde oplossingen wordt mobility management van MNs op de netwerklaag afgehandeld. Dit biedt transparantie t.a.v. veranderingen in hogere lagen, en zorgt er o.a. voor dat MNs niet op de hoogte behoeven te zijn van de betreffende functionaliteit. Deze methodes vereisen echter wel enkele nieuwe functionaliteiten en componenten in het netwerk, alsmede aanpassingen in de infrastructuur. De derde methode is gebaseerd op MultipathTCP (MPTCP), waarbij meerdere IP adressen voor een enkele data stream gebruikt kunnen worden, en de zgn. break-before-make-optie voor de afhandeling van de mobiliteit van MNs benut kan worden. Hiermee blijft het netwerk zelf en de infrastructuur ongemoeid en wordt het gehele mobility management in de transportlaag op de eindsystemen geïmplementeerd. MPTCP wordt ondersteund op slimme mobiele apparaten vanaf iOS versie 7 en Android versie 4.4. Het op grote schaal uitrollen van deze oplossing is daarmee haalbaar, met minimale 
overhead en lage complexiteit. Afsluitend vergelijken we de drie voorgestelde oplossingen en zetten de voor en nadelen van iedere oplossing tegen elkaar af. 



\section{Contents}

1 Introduction 4

1.1 Background . . . . . . . . . . . . . . . . 4

1.2 Problem Statement . . . . . . . . . . . . . . . . 6

1.3 Research Goal, Questions and Approach . . . . . . . . . . . 7

1.4 Thesis Organization . . . . . . . . . . . . . . . . 14

2 Future Mobile Network $\quad 20$

2.1 Centralized (current) LTE Network . . . . . . . . . . . . . . . . 22

2.2 Mobility Management in the Centralized LTE Network . . . . . . 23

2.3 Decentralized LTE Network . . . . . . . . . . . . . . . . 25

2.4 Mobility Management in a Decentralized LTE Network . . . . . . 26

2.5 Summary . . . . . . . . . . . . . . . . . . 31

3 Quantitative Comparison of the Centralized and Decentralized LTE Network Architectures $\quad 34$

3.1 Related Work . . . . . . . . . . . . . . . . . 36

3.2 LTE's Data and Control Planes . . . . . . . . . . . . . . . 37

3.3 LTE's Mobility Management Messages . . . . . . . . . . . . . . . 39

3.4 Hybrid Simulation and Analytical Modeling . . . . . . . . . . . . 42

3.5 Calculation of the Performance Metrics . . . . . . . . . . . 47

3.6 Numerical Results . . . . . . . . . . . . . . . . . . . . . 56

3.7 Conclusions . . . . . . . . . . . . . . . 63

4 Network Layer Approach, NAT-based Solution $\quad 66$

4.1 Related Work . . . . . . . . . . . . . . . . . 68

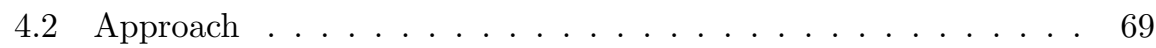

4.3 Architecture . . . . . . . . . . . . . . . . . 70

4.4 Setup of Simulation Study . . . . . . . . . . . . . . . . . . . . . . . . . . . .

4.5 Simulation Parameters . . . . . . . . . . . . . . . . . . . . . 81

4.6 Performance Evaluation . . . . . . . . . . . . . . . 83

4.7 Concluding Remarks . . . . . . . . . . . . . . . . . . . 87 
5 Network Layer Approach, SDN-based Solution 9

5.1 Related Work . . . . . . . . . . . . . . . . . . . . . . . . 92

5.2 Approach . . . . . . . . . . . . . . . . . . . . 93

5.3 Architecture . . . . . . . . . . . . . . . . . . . 94

5.4 Setup of Simulation Study . . . . . . . . . . . . . . . . . . 102

5.5 Simulation Parameters . . . . . . . . . . . . . . . . . . 103

5.6 Performance Evaluation . . . . . . . . . . . . . . . . . . . . 104

5.7 Concluding Remarks . . . . . . . . . . . . . . . . . . . . 108

6 Transport Layer Approach, MPTCP-based Solution 112

6.1 Related Work . . . . . . . . . . . . . . . . . . . . . . . . . 114

6.2 Approach . . . . . . . . . . . . . . . . . . . 115

6.3 Architecture . . . . . . . . . . . . . . . . . . . . 117

6.4 Setup of Simulation Study . . . . . . . . . . . . . . . . . . 123

6.5 Simulation Parameters . . . . . . . . . . . . . . . . . . . . . 124

6.6 Performance Evaluation . . . . . . . . . . . . . . . . . 125

6.7 Concluding Remarks . . . . . . . . . . . . . . . . . . . . . . 129

7 Conclusions and Directions for Future Research 132

7.1 Conclusions . . . . . . . . . . . . . . . . . . . . . . . 134

7.2 Summary of Contributions . . . . . . . . . . . . . . . . . . 139

7.3 Future Research . . . . . . . . . . . . . . . . . . . . . . 141

A Detailed Calculation of The Performance Metrics 144

A.1 Average Latency of Data Packet Delivery for an MN . . . . . . 144

A.2 Average processing Cost of Data Packet Delivery for an MN . . 146

B Open Data Management 150

$\begin{array}{ll}\text { Bibliography } & 150\end{array}$

$\begin{array}{ll}\text { Glossary } & 161\end{array}$

$\begin{array}{ll}\text { List of Publications } & 165\end{array}$ 




\section{Introduction}

This chapter provides background information and motivates the research of this Ph.D. thesis, details our research goal and questions, and presents the thesis structure.

\subsection{Background}

Over the last years, with the ubiquitous deployment and rapid evolution of mobile networks (e.g., 3GPP and WiMAX), offering continuous reachability, mobile users' demand for Internet access has been soared dramatically. The mobile devices (e.g., smart-phones and tablets) become an integral part of everyone's daily life and generate a substantial part of the total Internet traffic, which is still increasing significantly.

It is forecasted that by 2021 there will be around 12 billion mobile devices worldwide, from which $82 \%$ would be smart mobile devices generating up to $99 \%$ of all mobile data traffic. Overall, mobile data traffic is expected to increase 1013 fold, between 2016 and 2021 [15, 14]. Coping with such a demand in the current mobile networks might be neither economically nor technically viable. The Radio Access Network (RAN) of mobile networks cannot be easily extended due to the spectrum limitation. Furthermore, the architecture of core networks is highly centralized, which introduces scalability and reliability problems.

Mobile network operators augment the RAN capacity by improving spectrum utilization in several ways, e.g., by deployment of small cells, selectively offloading traffic from cellular access to WiFi technology, and exploiting multicarrier techniques or multiple Radio Access Technology (multi-RAT) approaches $[96,13]$.

The major challenge related to the core networks architecture (standardized by e.g., 3GPP, IETF) rises particularly from the fact that it is structured in a hierarchical and centralized way, covering multiple connected RANs. The core network hosts a few high level network entities, entitled anchor points, that handle both the Data plane (e.g., IP gateway, routing, forwarding, tunneling) and the Control plane (e.g., IP addressing, mobility management, monitoring) 
functions. These components also support other tasks related to e.g., QoS, security, and charging.

The anchor points, generally are placed at Internet exchange points to connect the mobile networks to external data networks e.g., the Internet. Therefore, in the data plane, the Mobile Nodes' (MNs') data traffic must traverse through both the RAN and the core network to be routed to and from the Corresponding service Node $(\mathrm{CN})^{1}$ through the central anchor points, see Fig. 1.1. In such an architecture, the anchor points also manage centrally the MNs' connectivity and mobility and maintain the reachability of the IP addresses allocated to the MNs after their movements. Moreover, the existing mobility management protocols are designed to be always active, servicing all the attached nodes and related traffic in the same way, even for motionless nodes that do not need mobility support. These all make the network prone to several limitations, e.g., sub-optimal routing, low scalability, signaling overhead, and bottlenecks/single point of failure issues $[30,35,31]$.

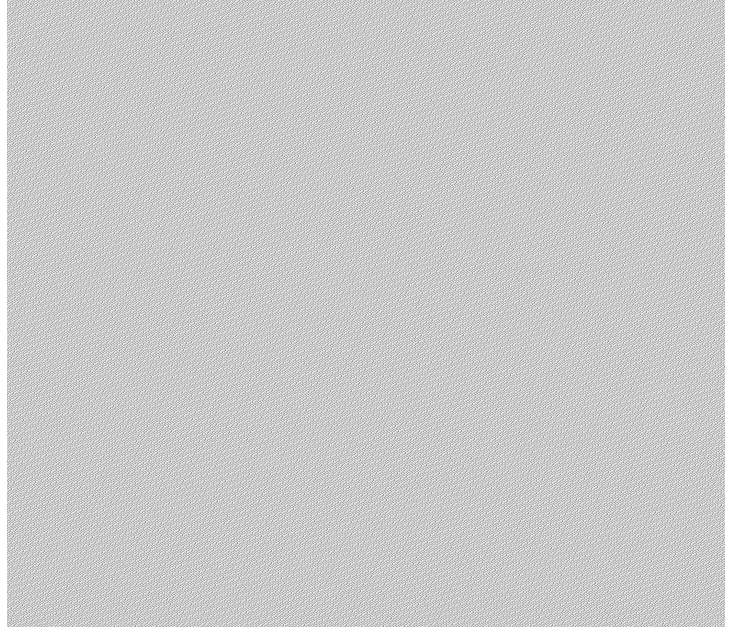

Figure 1.1: A general view of the current (centralized) mobile network architectures.

The straightforward solution to cope with the core network issues may consist of operators investment to upgrade the resources of the core network entities. Although this approach is technically feasible, network operators always prefer the cost-effective and long-term solutions.

\footnotetext{
${ }^{1} \mathrm{CN}$ is a node located within a fixed or mobile network and communicates with one or more mobile node(s).
} 
Traffic offloading is an alternative approach to mitigate the traffic overhead on the limited resources of the core network. This can be achieved by placing small-scale anchor points in the proximity of the access network to locally handle MNs connections and traffic [93]. This essentially leads to a decentralized network architecture deployment, see Fig. 1.2. This allows a MN's data traffic to be managed and handled via optimal usage of network resources, particularly when $\mathrm{MN}$ and $\mathrm{CN}$ are close to each other, but are both far from the anchor point $[31,30,35]$.

Even though decentralization also requires further investments for network architecture changes and management, on the long-term it is more cost-efficient than continuously extending capacity of the centralized architecture to cope with the ever-growing future demands.

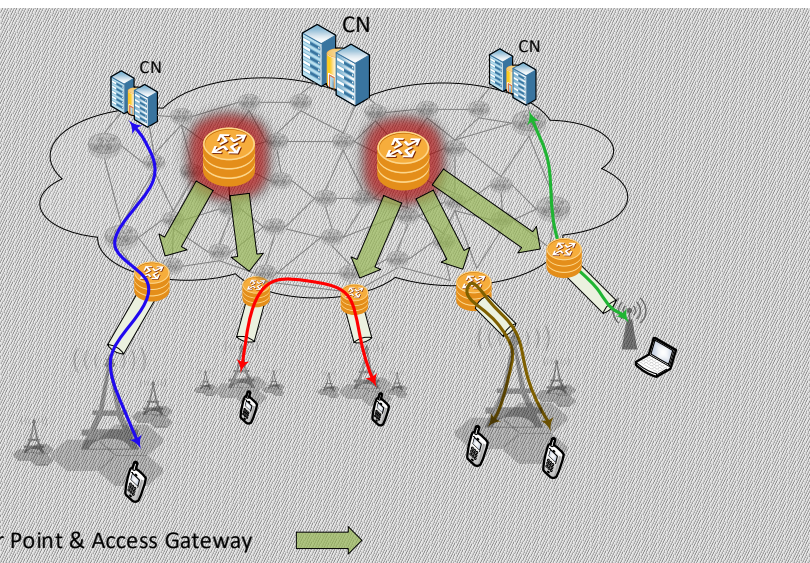

Figure 1.2: A general view of the decentralized mobile network architectures.

\subsection{Problem Statement}

Mobility management is an essential function in mobile networks, consisting of a set of procedures to: (i) provide reachability for the MNs roaming within the network; (ii) handle session continuity to MNs' ongoing data traffic during movement. The existing mobility management solutions, e.g., MIPv6 [64], PMIPv6 [49], and GTP [22], rely on a centralized anchor point, which is also in charge of the other data and control planes related tasks. Accordingly, the centrally managed IP traffic mobility approaches can not scalable enough to efficiently deal with demands raised by ever-growing number of mobile users of new generation of applications seeking for traffic session continuity. 
Following a decentralized architecture, instead of aggregating traffic at the central anchor point, MNs' IP flows and mobility related functions are split among the distributed anchor points, being placed closer to edges of the access network. By doing so, the potential congestion in core nodes and the related links is reduced, and better-suited data paths can be established for the IP flows by using standard routing algorithms.

Problem Statement: In the decentralized architecture, relocation of the MNs' mobility anchor will happen far more often. Therefore, it demands additional mechanisms to be implemented above the distributed anchor points, to preserve the $M N s$ ' traffic flow(s) active, by enabling sessions continuity to the mobile devices and steering the data packets towards the new anchor points, see Fig. 1.3.

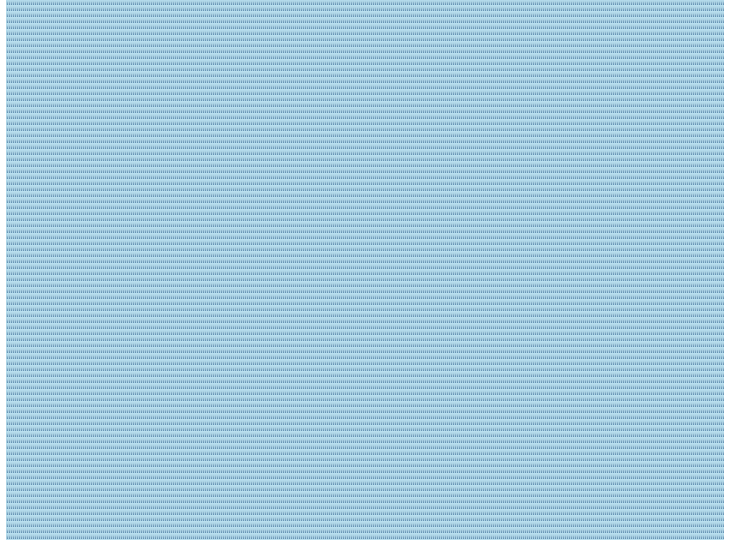

Anchor Point \& Access Gateway

Figure 1.3: Looking for mechanisms to preserve the MNs' active flow(s) during anchor points relocation.

\subsection{Research Goal, Questions and Approach}

\subsubsection{Goal}

LTE is expected to be the leading mobile networking technology, handling a substantial part of the global mobile data traffic in the coming decade. It is predicted that the development of LTE will not keep up with the growth of mobile traffic, and while it supports almost $69 \%$ (out of 7.5 Exabytes) of the 
current mobile traffic, it is estimated to handle about $79 \%$ (out of 50 Exabytes) of the worldwide mobile data traffic by $2021[15,14]$. Therefore, in this thesis we pay special attention to the LTE system, and investigate an architecture for realizing a decentralized LTE network to deal with the rapid growth of mobile data traffic.

In particulate, we define the overall goal of this thesis as follows:

Research Goal: Designing novel approaches to support IP traffic session continuity for moving MNs in a decentralized LTE network architecture.

LTE defines a unique core network, entitled as Evolved Packet Core (EPC), providing various IP-based services over multiple wireless and fixed access technologies. The existing LTE system specification allows a MN to interconnect the EPC through the 3GPP (e.g., 2G, 3G, 4G) and non-3GPP (e.g., WiFi, WiMAX) radio access network technologies. Non-3GPP access can be split into the trusted and untrusted access categories. In the trusted access, the MN interacts directly with the EPC. In the untrusted access, MN needs to communicate with the LTE core network via an entity called the Evolved Packet Data Gateway (ePDG). The main role of the ePDG is to provide security mechanisms (e.g., IPsec tunnelling) for MN's connection, over an untrusted non-3GPP access $[21]$.

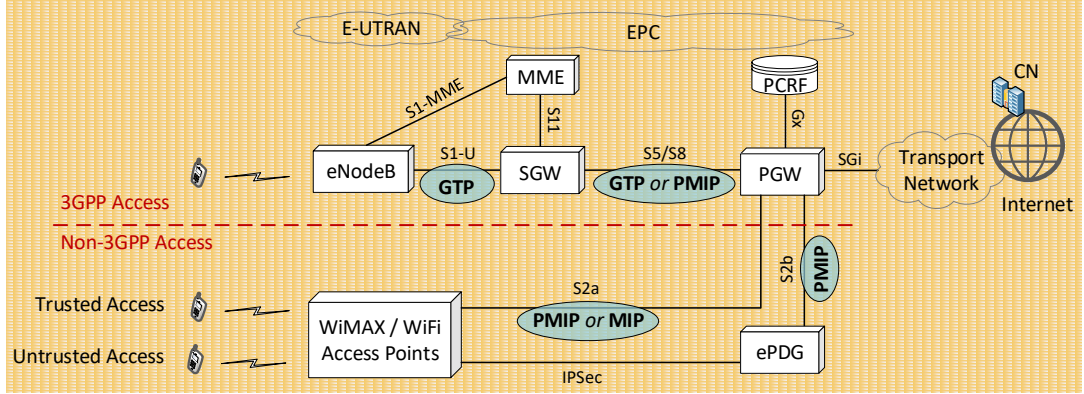

Figure 1.4: LTE system radio access network technologies.

Depending on the type of the access network and links that are involved during the MN's attachment and handover procedures, various mobility management protocols with different functionalities may be applied, see Fig. 1.4. The most widely deployed of LTE networks are based on the 3GPP standards and the majority of mobile users make data connections through the $3 \mathrm{GPP}$ access network. Hence, in this thesis we particularly focus on developing novel 
mobility support mechanisms for the MNs connecting to a decentralization LTE network via $3 \mathrm{GPP}$ access network.

\subsubsection{Research Questions and Approach}

As described in $\S 1.1$, decentralization of the mobile networks architecture is a promising approach to cope with the MNs' data traffic demands in the future. Given that in this thesis we focus on the LTE network, we define our first research question as:

RQ-1: How can the current LTE network architecture be modified to realize a decentralized LTE system?

We address the RQ-1 in Chapter 2 by giving a brief background about the current LTE system and its mobility management mechanisms for 3GPP access. Next, we investigate an approach combining the Packet Data Network Gateway (PGW) functions with the Serving Gateways (SGWs), named as S/PGWs, to realize a decentralized LTE network deployment.

In the existing 3GPP LTE standard (with the centralized architecture) IP traffic continuity is not supported when a MN changes its anchor point (PGW), e.g., during inter-operator roaming procedure. Currently, MN's traffic remains anchored to a PGW until it moves out of the access network. If the $\mathrm{MN}$ is handed over to a different PGW, the flows initiated at the previous one will be stopped. This is due to the fact that, in the current 3GPP technical specifications, there is no standard mechanism to support a handover procedure with PGW relocation.

Following the decentralized architecture, the mobility anchor points (S/PGWs) are placed closer to the edge network and the MNs are expected to change their traffic anchor point far more often. The main obstacle to implement IP traffic continuity in the decentralized architecture is raised by the fact that in the current LTE standard, there is neither a signaling nor a data forwarding scheme available between two different anchor point entities (PGWs). In Chapter 2, we also discuss the required modifications in the current LTE's data and control planes to be used for supporting the MN's mobility and handover among the S/PGWs in the LTE network with decentralized architecture. We extend the NS3-LENA [3] simulation environment to implement and evaluate the functionality of the proposed approach.

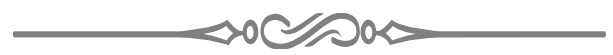

The core architecture of the current LTE system cannot be scaled well to cope with the future traffic demands, owing to its highly centralized and hierarchical composition. Decentralization of the LTE network architecture would be a 
promising approach to deal with the future mobile data traffic. However, this strategy has not been properly examined through performance studies, and in particular the gain of such an approach on the LTE network efficiency is an open question. Therefore, in order to come up with an estimation how the future traffic can actually benefit from the LTE architecture decentralization, we define our second research question as:

RQ-2: To which extent can decentralization of the network architecture improve efficiency and scalability of the LTE system?

RQ-2 is addressed in Chapter 3 where we conduct a study to evaluate a decentralized LTE architecture, quantifying the gains on performance and scalability as compared to a centralized architecture. Our proposed approach combines simulation and analytical modeling, enabling relatively detailed analysis of the performance of the network architecture, while keeping computation complexity limited. Regarding the performance indicators, we look particularly at the latency and the processing cost for data packet delivery, MN's initial attachment procedure, and handover procedure. Further, we look at the load imposed by the data and control planes over the network routers and links. We used MAT$\mathrm{LAB}$ as the environment to implement the models and perform the comparative analysis.

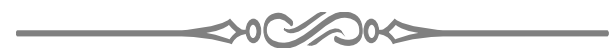

As stated in $\S 1.3 .1$ in a decentralized architecture, the anchor points are distributed closer to edges of the access network, anchoring the MNs attached locally. Therefore, an additional mechanism need to handle MNs' mobility, performing handover with an anchor point relocation. The approaches to support IP traffic session continuity for the MNs moving in a network with distributed anchor points, may target different layers of the protocol stack. The techniques based on the Network and Transport layers are the most widely studied e.g., $[46,54,83,36,37,59,55,78]$. Therefore, in RQ-3, 4 \& 5 we follow on such techniques.

Generally, the mobility management solutions corresponding to the network layer handle MN's mobility in a transparent manner and hide any changes from the upper layers. This leads us to the third research question, defined as:

RQ-3: How can current network layer technologies such as Network Address Translation (NAT) be applied to implement the MN's mobility related functions in a decentralized LTE network architecture? 
We address the RQ-3 in Chapter 4 . We develop an approach relying on the NAT technique, which is widely used in the current Internet. Therefore, its implementation using the existing technology and integration with the already implemented network, being feasible with minimal overhead and complexity. This makes the solution to be handily deployable and scalable. This approach introduces two new NAT routers at the edges of the transport network to carry out the correct translation between MN's IP addresses, assigned before and after handover by the corresponding S/PGWs, to redirect the traffic flow to the MN's new position. We extend the NS3-LENA simulation environment to implement and evaluate the functionality and performance of our NAT-based approach.

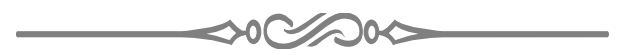

The next generation of the mobile networks (e.g., $5 \mathrm{G})$ are supposed to provide massive capacity and connectivity with a powerful heterogeneous infrastructure. They should be capable of handling a variety of complex functions on supporting diverse sets of new and unforeseen services, users, and applications. To address these key challenges, there is a need to enhance the network intelligence as a mean for realizing a successful and powerful network infrastructure. Network Virtualization (NV) and Network Function Virtualization (NFV) are the principles to help the network operators for speeding up their service innovations, and supporting the requirements for the future demands. In this regards, Software Defined Network (SDN) is a promising enabler, playing a crucial role in design of the future wireless networks e.g., [26, 94]. Therefore, we define our fourth research question as:

RQ-4: How can future network layer technologies such as SDN be applied to implement the MN's mobility related functions in a decentralized LTE network architecture?

We address the RQ-4 in Chapter 5. In that chapter, we benefit from the capability of SDN on detaching the control plane from data plane and propose two solutions to support MNs' mobility in a decentralized LTE network. OpenFlow [7], is the most common communication protocol used in the SDN, that significantly facilitates traffic management by accessing the control plane and data plane of SDN-based switches over the network. Our solutions are different, based on using the OpenFlow-only or OpenFlow-hybrid switches for the implantation. In the first solution, the SDN Controller makes the forwarding decision for every packet in the network and the incoming data traffic always goes through the switch's flow-tables pipeline. The solution is more suited for the networks that fully rely on SDN-based switches. In the latter solution, based on OpenFlowhybrid switches, only the switches placed at the edges of the transport network 
must be managed by the SDN Controller and the rest can be replaced by the typical L3 routers. Therefore, implementation of the second solution is easily feasible with a minimum overhead and complexity. We extend the NS3-LENA simulation environment to implement and evaluate the functionality and performance of the proposed SDN-based solutions. Having said that, SDN is going to be a key technique to efficiently address the demands of the future mobile networks, the proposed solutions will be readily feasible to apply in the next generation of the network's infrastructures.

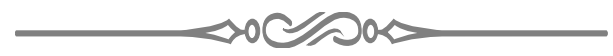

Transport layer mobility support schemes keep the network infrastructure intact and implement the whole functionality for handling MNs' mobility in the transport layer of only the end host entities. Benefiting from this advantage of the transport layer-based solutions and given the popularity of TCP as the most widely used protocol in todays Internet e.g., $[58,11]$, our fifth research question is defined as:

RQ-5: How can transport layer technologies such as Multipath-TCP (MPTCP) be applied to implement the MN's mobility related functions in a decentralized LTE network architecture?

We address the RQ-5 in Chapter 6 , where we propose a solution based on the MPTCP, which is also one of the key candidate protocols to efficiently address demands for faster connectivity, higher download rate and more reliable communication in the future mobile networks (e.g., 5G) $[27,51,24]$. In that chapter, we benefit from the MPTCP protocol feature on supporting multiple IP addresses for a single data-stream and its break-before-make option to develop our last solution for handling the MNs' handover procedure between different S/PGWs in the decentralized LTE network. The proposed solution can manage MNs' mobility without demanding an additional network interface and uses only one 3GPP interface on MNs. We extend the NS3-LENA simulation environment to implement and evaluate the functionality and performance of the MPTCP-based solution. MPTCP has been already deployed on the Apple smart phones and tablets (since iOS 7) to improve the performance of delay-sensitive applications (e.g., Siri) [12], and it is also available for the smart-devices running Android 4.4 [4]. This makes the proposed approach easily feasible to be implemented at a large scale with minimal overhead and complexity.

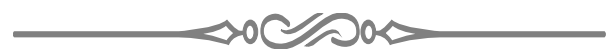


Fig. 1.5 presents the organization of our studies according to the defined research questions to achieve the main goal of this thesis. By answering the RQ-1, first we present an approach to realize an LTE network with decentralized architecture deployment, as a sustainable approach to deal with demands for mobile data traffic in the near future. We use the new LTE architecture as the platform to develop and integrate our mobility management solutions proposed in the RQ-3, 4 and 5 .

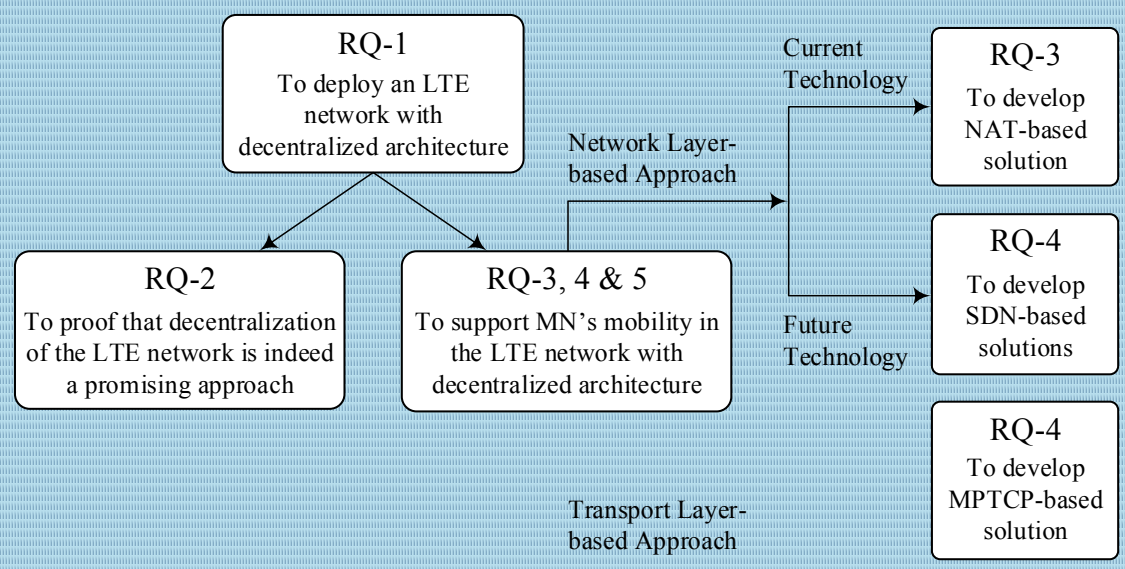

Figure 1.5: Organization of our research according to the research questions.

Next, in RQ-2, we conduct an assessment of the network efficiency and scalability, in terms of various data and control planes parameters, in the current and the proposed decentralized LTE network architectures. The aim of this study is to show how much the network decentralization can improve performance of the LTE system and accordingly to proof that this approach is a necessity to be followed in the LTE network to deal with the future demands. The outcomes of these research question can provide the mobile network operators an initial insight on decision making and policy development procedures to accommodate the network infrastructure for coping with the future data traffic usage.

Finally, by answering the RQ-3, 4 and 5, we develop several solutions in order to handle traffic session continuity for the MNs, roaming in the LTE network with decentralized architecture. The results of the last three research questions provide mobile network operators the opportunity, by considering e.g., the type of application, the technology applied for the implantation of network infrastructure, and the overhead and complexity, to choose the most appropriate approach, fulfilling their requirements. 


\subsection{Thesis Organization}

In the following we provide the relationship and a short summary of each chapter in the remainder of this thesis. In addition, we identify the publications used as the basis for each chapter. Fig. 1.6 presents the relation between the chapters of this thesis.

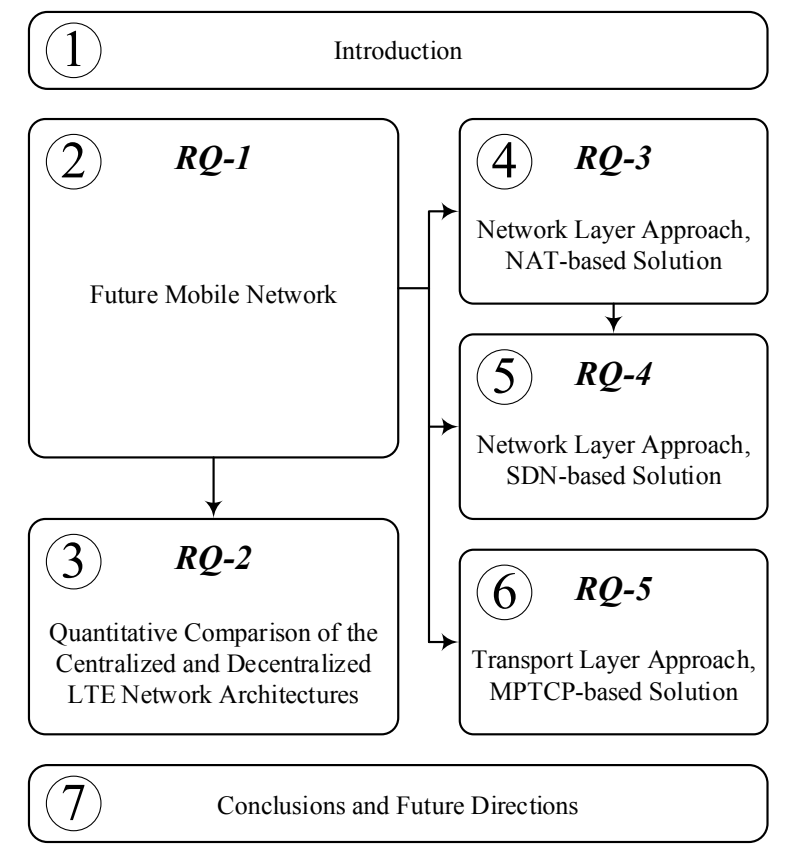

Figure 1.6: Thesis's organization.

\section{Chapter 2: Future Mobile Network}

In Chapter 2, first we provide a brief background about the current LTE system and its mobility management mechanisms for the 3GPP access. These are essential for understanding the reminder of this thesis. Second, we describe an approach to develop a decentralized LTE network architecture. In a decentralized architecture, relocation of the MNs' mobility anchors will happen far more often. In such case, two levels of mobility support mechanisms are required to handle traffic session continuity for the MNs performing a handover with an anchor point relocation: (i) within the EPC network; and (ii) above the 
EPC network, which hosts the MNs' corresponding services. Third, we describe the necessary modifications in the current EPC data and control planes to be used to support MNs' mobility within the EPC network of the decentralized LTE architecture. The mechanisms to handle the MNs' mobility above the EPC network are presented in Chapter 4, 5 and 6.

The content of Chapter 2 is partially based on the following publications:

- M. Karimzadeh, L. Valtulina, A. Pras, J.L. van den Berg, R. de O. Schmidt, M. Liebsch and T. Taleb, Double-NAT Based Mobility Management for Future LTE Networks, In Proceedings of the IEEE Wireless Communications and Networking Conference (WCNC), 2017 [69].

- M. Karimzadeh, L. Valtulina, J.L. van den Berg, A. Pras, P. Garrido Ortiz and R. Sadre, MultiPath TCP to Support User's Mobility in Future LTE Network, In Proceedings of the IFIP/IEEE Wireless and Mobile Networking Conference (WMNC), 2017 [70].

- M. Karimzadeh, J.L. van den Berg, R. de O. Schmidt and A. Pras, Quantitative Comparison of the Efficiency and Scalability of the Current and Future LTE Network Architectures, Journal of Wireless Communications and Mobile Computing (WCMC), 2017 [72].

\section{Chapter 3: Quantitative Comparison of the Centralized and Decen- tralized LTE Network Architectures}

A major problem of the current LTE system is the fact that its data and control plane procedures are highly hierarchical, demanding management of several tunnels between a central anchor point and MNs. This makes the network prone to e.g., sub-optimal routing, low scalability, and signaling overhead issues. This hierarchy can be eliminated by decentralization of the LTE core network functions, leading to anchor points distributed closer to edges of the access network. As a results, MNs' connection functions, data traffic, and mobility are managed locally. Although, decentralization of the LTE network architecture seems, on the long term, to be a cost-effective and also sustainable approach to effectively reduce the load on the core network entities, its gain on the network performance and efficiency is an open question. Therefore, in Chapter 3 we: (i) investigate in detail the GTP and PMIP protocols' data and control planes related functions (e.g., tunneling and messaging); (ii) conduct a hybrid study, combining simulation and analytical modeling, in order to evaluate the performance and scalability of the current and a decentralized LTE network architecture, based on GTP and PMIP protocols. Particularly, in this chapter we aim to quantify and analyze the impact of the number of 
connected MNs to the network, on the performance and scalability of LTE with different network architectures.

The content of Chapter 3 is based on the following publication:

- M. Karimzadeh, J.L. van den Berg, R. de O. Schmidt and A. Pras, Quantitative Comparison of the Efficiency and Scalability of the Current and Future LTE Network Architectures, Journal of Wireless Communications and Mobile Computing (WCMC), 2017 [72].

\section{Chapter 4: Network Layer Approach, NAT-based Solution}

In a decentralized LTE network, during a handover procedure with anchor points relocation, the MNs' IP traffic forwarding and session continuity within the EPC network can be managed by the mechanism presented in Chapter 2. In Chapter 4, we discuss an approach to handle MNs' mobility above the EPC network. The proposed solution is based on the NAT technique, which is a widely used technique in today's Internet. Therefore, integration of NAT functions into several routers of an already deployed network is easily feasible with a minimal overhead and complexity. This makes the solution to be readily deployable at a large scale.

The content of Chapter 4 has been publish in:

- M. Karimzadeh, L. Valtulina, A. Pras, J.L. van den Berg, R. de O. Schmidt, M. Liebsch and T. Taleb, Double-NAT Based Mobility Management for Future LTE Networks, In Proceedings of the IEEE Wireless Communications and Networking Conference (WCNC), 2017 [69].

\section{Chapter 5: Network Layer Approach, SDN-based Solution}

Future mobile networks (e.g., 5G) need to be more flexible and dynamic, and easily manageable to accommodate the expected demand. SDN has inherent capabilities allowing to; (i) detach the control and data planes enabling the network to be centrally manageable; (ii) bundle all traffic (e.g., IP based, Ethernet or lower layers) as flows; and (iii) update traffic forwarding rules dynamically, providing supreme flexibility to network management. This make the SDN paradigm well suited to build mobile networks that are highly scalable and readily adaptable to cope with new services and applications demands. Motivated by the increasing popularity of SDN technology as well as playing a key role for the future generation of mobile networks, in Chapter 5 we present two new approaches, tacking advantages of the capabilities offered by SDN, to handle the MNs' mobility above the EPC network. Our proposed solutions 
differ in the sense that they use the OpenFlow-hybrid or OpenFlow-only switches for the implementation.

The content of Chapter 5 is based on the following publications:

- M. Karimzadeh, L. Valtulina and G. Karagiannis, Applying SDN/OpenFlow in Virtualized LTE to support Distributed Mobility Management (DMM), In Proceedings of the 4th International Conference on Cloud Computing and Services Science (CLOSER), 2014 [68].

- M. Karimzadeh, A. Sperotto and A. Pras, Software Defined Networking to Improve Mobility Management Performance, In Proceedings of the International Conference on Autonomous Infrastructure, Management, and Security (AIMS), 2014 [67].

- M. Karimzadeh, L. Valtulina, J.L. van den Berg, A. Pras, M. Liebsch and T. Taleb, Software Defined Networking to Support IP Address Mobility in Future LTE Network, In Proceedings of the IFIP/IEEE Wireless Days (WD) Confeence, 2017 [71].

\section{Chapter 6: Transport Layer Approach, MPTCP-based Solution}

Motivated by the fact that: (i) the TCP-based applications are the most popular ones in today's Internet [58]; (ii) MPTCP is a key protocol to efficiently address the demands on the future mobile networks [27, 51, 24]; and (iii) MPTCP being available for the smart-devices running iOS and Android [12, 4], in Chapter 6 we present a MPTCP-based scheme to address MNs' mobility issues in the decentralized LTE architecture. The proposed solution can handle the MNs' mobility without demanding an additional network interface on the MNs, and with a minimal impact on the existing network implementation.

The content of Chapter 6 is based on the following publications:

- M. Karimzadeh, L. Valtulina, J.L. van den Berg, A. Pras, P. Garrido Ortiz and R. Sadre, MultiPath TCP to Support User's Mobility in Future LTE Network, In Proceedings of the IFIP/IEEE Wireless and Mobile Networking Conference (WMNC), 2017 [70].

\section{Chapter 7: Conclusions and Directions for Future Research}

In Chapter 7, we summarize our main findings and contributions. We discuss outcomes of the quantitative comparison performed on the LTE network with different architectures. We also compare our proposed MN's mobility 
support approaches according to their respective ease-of-implementation, performance, scalability and overhead. Furthermore, we indicate directions for future work. 



\section{Future Mobile Network}

In this chapter we give a brief overview about the current LTE network and its mobility management mechanisms for $3 G P P$ access. These are essential for understanding the problems statement being addressed in this thesis. Next, we present an approach to realize a decentralized LTE network deployment and detail the essential modifications in the current LTE data and control planes to be used for supporting MNs' mobility within LTE new architecture. The publications related to this chapter are [69, 70, 72].
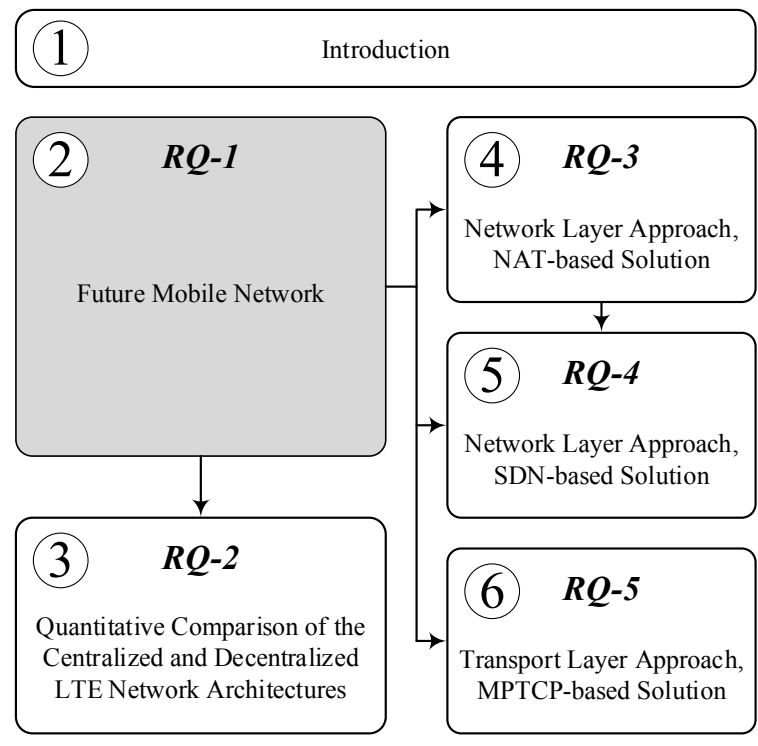

(7) Conclusions and Future Directions


This chapter is organized as follows:

- Section 2.1 gives a brief overview of the current LTE network system as far as relevant to this thesis.

- Section 2.2 describes the mobility management mechanisms in the current LTE network.

- Section 2.3 presents an architecture to realize an LTE network with decentralized deployment and states the issues on supporting the MN's mobility in the LTE new architecture.

- Section 2.4 details the essential modifications in the current LTE system data and control planes to be used to support MNs' mobility within the LTE network with decentralized architecture.

- Section 2.5 summarizes this chapter. 


\subsection{Centralized (current) LTE Network}

This section gives a brief overview of the current LTE system architecture with the main network elements and interfaces relevant to this thesis. These are principal for comprehending the studies being performed in the next chapters.

The existing LTE network architecture is hierarchical and defines the Evolved Packet System (EPS) consisting of Evolved Universal Terrestrial RAN (E-UTRAN) and EPC, see Fig. 2.1. The E-UTRAN consists of a network of radio base stations, evolved NodeBs (eNodeBs), that provides radio connectivity to the MNs. The EPC is a multi-access IP-based network that uses a common core network for the $3 \mathrm{GPP}$ and non-3GPP radio access, and fixed access.

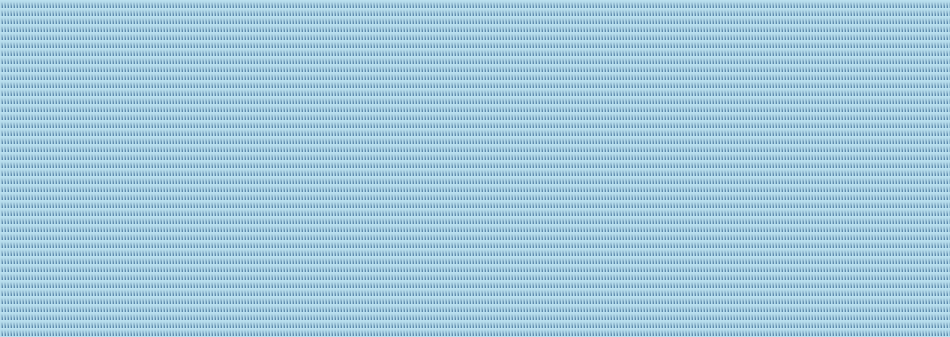

Figure 2.1: Current LTE system architecture and its mobility management protocols for $3 \mathrm{GPP}$ access.

The EPC consists of four main elements (Fig. 2.1), that allow for the convergence of packet-based services [21]:

- The Packet Data Network Gateway (PGW), which provides connections between the EPC and other external IP networks (e.g., IMS and Internet), and carries out several additional functions, such as IP address anchoring and allocation, traffic routing, packet filtering, monitoring, policy control and charging.

- The Serving Gateway (SGW), which is a user-plane node that provides data paths and routes traffic between the eNodeBs and PGW. It also acts as a local mobility anchor for MNs performing handover between eNodeBs.

- The Mobility Management Entity (MME), whose key role is to process the signaling between the MN and core network. The main functions supported by the MME can be classified as the functions related to: (i) bearer management e.g., the establishment, maintenance and release of the bearers; and (ii) connection management e.g., assign network resources, support mobility, roaming and handover procedure. 


\subsection{MOBILITY MANAGEMENT IN THE CENTRALIZED LTE NETWORK}

- The Policy and Charging Rule Function (PCRF), which dynamically controls and manages all data sessions, and determines quality of service (QoS) policies and charging rules to SGW (if PMIP is used) and PGW (if GTP is used).

To provide data services, EPS network uses couple of logical connections between the MN and EPC, called as EPS bearers. An EPS bearer consists of several tunnels between different network elements, over which data packets are transferred using IP protocol. During a MN's attach procedure three types of bearers are set up in order to transport MN's traffic between the EPS entities; Radio bearer, $S 1$ bearer and S5/S8 bearer (Fig. 2.2).

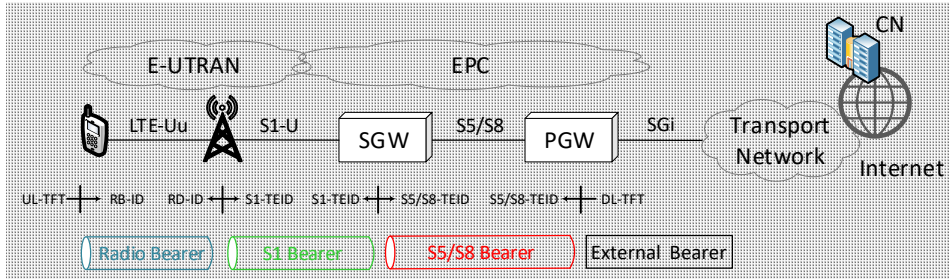

Figure 2.2: EPS bearers in the current LTE network.

The S1 and S5/S8 bearers use the GTP protocol to identify the individual connections between two nodes. A Tunnel Endpoint Identifier (TEID) ${ }^{1}$ is assigned to each GTP bearer allowing the EPS nodes to determine to which specific bearer a particular packet belongs. Each EPS bearer is associated with one Traffic Flow Template (TFT), defining the filtering rules to differentiate data packets based on some characteristics e.g., applications, QoS requirements. A DL-TFT is created by the PCRF after fulfilling an IP-CAN Session Establishment procedure [20] by the PGW. Next, the PGW maps the (DL-TFT $\rightarrow$ S5/S8TEID) to the downlink traffic. IP data packets are tunneled between the PGW and SGW (over the S5/S8 interface) and between the SGW and eNodeB (over the $\mathrm{S} 1-\mathrm{U}$ interface), and next sent over the radio interface between the eNodeB and MN.

\subsection{Mobility Management in the Centralized LTE Network}

A mobility management mechanism provides a set of procedures to enable seamless IP traffic session continuity for moving MNs within the network. In this

\footnotetext{
${ }^{1}$ For the PMIP, a Generic Routing Encapsulation (GRE) key is used as a identifier.
} 
section we briefly describe the commonly being used mobility management solutions in the existing LTE network for 3GPP access. We will study in detail the control and data planes of this solutions to analyze the performance and scalability of the current and decentralized LTE network architectures, in Chapter 3.

Current EPC may use GTP or PMIP protocol to support MNs' mobility for $3 \mathrm{GPP}$ access networks [20], where the PGW acts as a central mobility anchor point. These protocols allow an uninterrupted handover for the MNs during intra-network mobility. To manage MNs mobility using GTP or PMIP, the PGW might connect to SGW via S5/S8 interfaces (Fig. 2.1) for non-roaming and roaming scenarios between different operators, respectively [20]. When a MN connects to an eNodeB, its traffic is anchored to the PGW (via the SGW) and is encapsulated in a GTP tunnel between the eNodeB and SGW and in another GTP (or PMIP) tunnel between the SGW and PGW.

When the MN performs a handover between two eNodeBs to keep the ongoing IP traffic flow(s) active, a new tunnel(s), only S1-U or S1-U \& S5/S8, are established between the EPC and E-UTRAN entities depending on whether the target eNodeB is served or not by the same SGW, see Fig. 2.3.

The procedure described above shows that the existing data plane and mobility management procedure are highly hierarchical, demanding management of several tunnels between the PGW and the MNs. In a large LTE network, the PGW needs to maintain a considerable number of (e.g., a range of millions for a nationwide network) per-user tunneling data, which may cause scalability and performance issues.

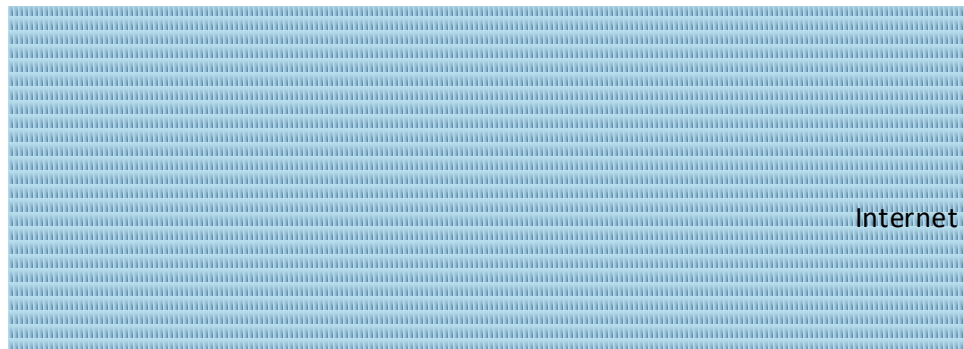

Figure 2.3: Date and control planes tunnel updating during MN's handover (with SGW relocation) in the current LTE system.

The GTP protocol is able to fully handle the control and data planes. It can forward the MNs downlink packets from source location to target place during handover. The PMIP, however can only handle MNs mobility and perform data forwarding after handover procedure. Moreover, it is not able to control the bearers and QoS signaling. When PMIP is used over the S5/S8 interface, the 
GTP bearers are only defined between MNs and the SGW. In this case, the SGW takes over the bearer binding operations, and an additional connection (dash line in Fig. 2.1) needs to be created between the SGW and PCRF to provide the required information on QoS policy [21, 43, 82].

A MN may perform a handover either in idle or active mode. In idle mode, the MN stays in power consumption mode and does not inform the network about the location information. The network uses the tracking and paging procedures to discover position of MN. In active mode, MN mobility is completely under control of the network. The decision to perform a handover and to choose the target cell is handled by the network, based on measurements performed by the eNodeB and the MN. During the handover procedure, GTP can locate the MN's position, even in the idle mode to establish the required data and control planes tunnels. However, PMIP does not support tracking and paging functions and the MN needs to be always in active mode. Therefore, GTP protocol is mostly used in the access network between SGW and eNodeBs to eliminate the aforementioned drawbacks of PMIP.

The decision for using GTP or PMIP over the S5/S8 interface depends on several parameters, such as technical support and existence of roaming scenarios among the 3GPP access and non-3GPP access networks (see also Fig. 1.4). Note also that mobility management protocols for non-3GPP, such as Mobile IP (MIP) and Dual Stack MIP (DSMIP) [21], are out of the scope of this thesis.

Local IP Access (LIPA) and Selected IP Traffic Offload (SIPTO) have been introduced in the 3GPP Release 10 to alleviate data traffic load on the LTE's core network [16]. LIPA enables IP connectivity between local MNs via an eNodeB without traversing traffic through the core network. SIPTO on the other hand, enables to offload certain types of traffic at the network entities that are geographically/topologically close to the MNs position. However, they are limited in supporting the MNs' mobility only for the local nodes [17].

\subsection{Decentralized LTE Network}

This section briefly presents an approach to realize an LTE network with decentralized architecture and states the main issues to be addressed for supporting the MN's mobility in the LTE system with new architecture.

The hierarchy of the data and control planes in the current LTE network can be eliminated by co-locating the SGW and PGW functions into a single entity, entitled as S/PGW, see e.g., [52, 97, 53, 77, 104]. Accordingly, the S/PGWs are distributed closer to edges of the network to handle the MNs' connection functions, data traffic, and mobility locally. This approach basically leads to deployment of an LTE network with decentralized architecture, that can effec- 
tively reduce the load on the core network entities (Fig. 2.4). In the current LTE network, MNs rarely change their attached PGW. They often remain attached to the same operator's access network and their corresponding traffic remain anchored to a single PGW, placed deeply in the core network.

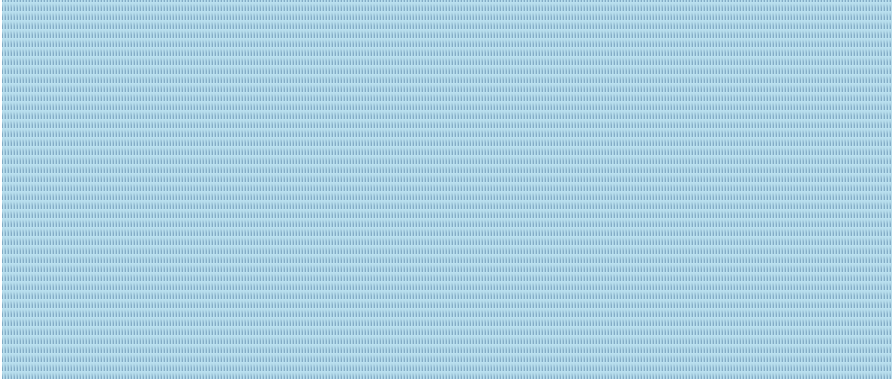

eNodeB-2

Figure 2.4: Decentralization of the LTE core network architecture.

Following the decentralization concept in the LTE system, relocation of the MNs' edge anchor points (S/PGWs) will happen far more often. In this case, two layers of mobility support mechanism are needed to handle the MNs' traffic session continuity, while they move among different S/PGWs:

- Inside the EPC network, in order to forward MNs' data packets between two eNodeBs served by different S/PGWs.

- Above the EPC network (on the transport network hosting the MNs' corresponding services), in order to redirect MNs' data traffic between the CNs and S/PGWs.

The following section describes the required modifications in the current LTE data and control planes to be used for supporting the MNs' mobility inside the EPC of the LTE network with decentralized architecture. Handling of the MN's mobility above the EPC network can be accomplished using one of the our developed mechanisms, presented in Chapters 4, 5 or 6 .

\subsection{Mobility Management in a Decentralized LTE Network}

In this section first we briefly describe limitation of the current LTE system on managing the MN's mobility during an anchor point (PGW) relocation. Next, 


\subsection{MOBILITY MANAGEMENT IN A DECENTRALIZED LTE NETWORK $\quad 27$}

present an approach that updates and modifies the existing LTE network procedure specified to handle MN's handover with SGW relocation, to be applied for supporting the MN's mobility inside the EPC of the LTE network with distributed anchor points (S/PGW), see $\S 2.3$.

IP-based traffic session continuity, upon changing the attached PGW, is not supported in the current 3GPP's LTE standard. The data and control planes of existing LTE system have been designed in hierarchical fashion, implying that the MN's traffic session remains anchored to a single PGW until it moves out of the access network served by the specific PGW. If an $\mathrm{MN}$ is handed over to another PGW, e.g., during inter-operator roaming, a PDN Disconnection procedure will be triggered by the network for the IP flow(s) initiated at the previous PGW. Next, the new PGW anchors the MN and serves the re-initiated traffic. This implies a disruption in the MN's ongoing traffic, since the MN's IP address is not maintained but a new one is assigned. This is due to the fact that, in the current 3GPP's technical specifications there is neither a signaling nor a data forwarding scheme available between two different PGWs, in order to handle the continuity of bearers after PGW relocation.

Following a decentralized architecture where the SGW and PGW functions are combined into a single entity, the existing control messages and traffic forwarding mechanisms specified in the current LTE standard and used for the MN's handover with a SGW relocation, can be revised and applied to support the migration of MN's bearers to the newly established data plane on the target $\mathrm{S} / \mathrm{PGW}$ in the LTE network with decentralized architecture. This can handle the use of same IP address of MN (allocated by the initial S/PGW) in the target $\mathrm{S} / \mathrm{PGW}$ and accordingly can realize a seamless handover procedure and traffic session continuity for an MN moving between two S/PGWs. This modification should enable the following functions:

(I) The target S/PGW needs to be informed to implement a GTP bearer for the moving MN. This bearer is used to serve the flow(s) kept active by the MN after handover.

(II) The source S/PGW needs to be informed when the IP address of the flow(s) kept active by the moving $\mathrm{MN}$, can be released and be assigned to another MN newly attached to the network.

In a decentralized EPC, due to the unification of SGW and PGW into a single entity, the S5/S8 bearer is not needed and direct mapping from External bearer to $\mathrm{S} 1$ bearer (DL-TFT $\rightarrow \mathrm{S} 1-\mathrm{TEID}$ ) can be performed in the S/PGW, see Fig. 2.2. 
In the current LTE standard, a procedure to adjust the S1-TEID in a X2based handover with SGW relocation ${ }^{2}$ is specified in $\S 5.5 .1 .1 .3$ of [20].

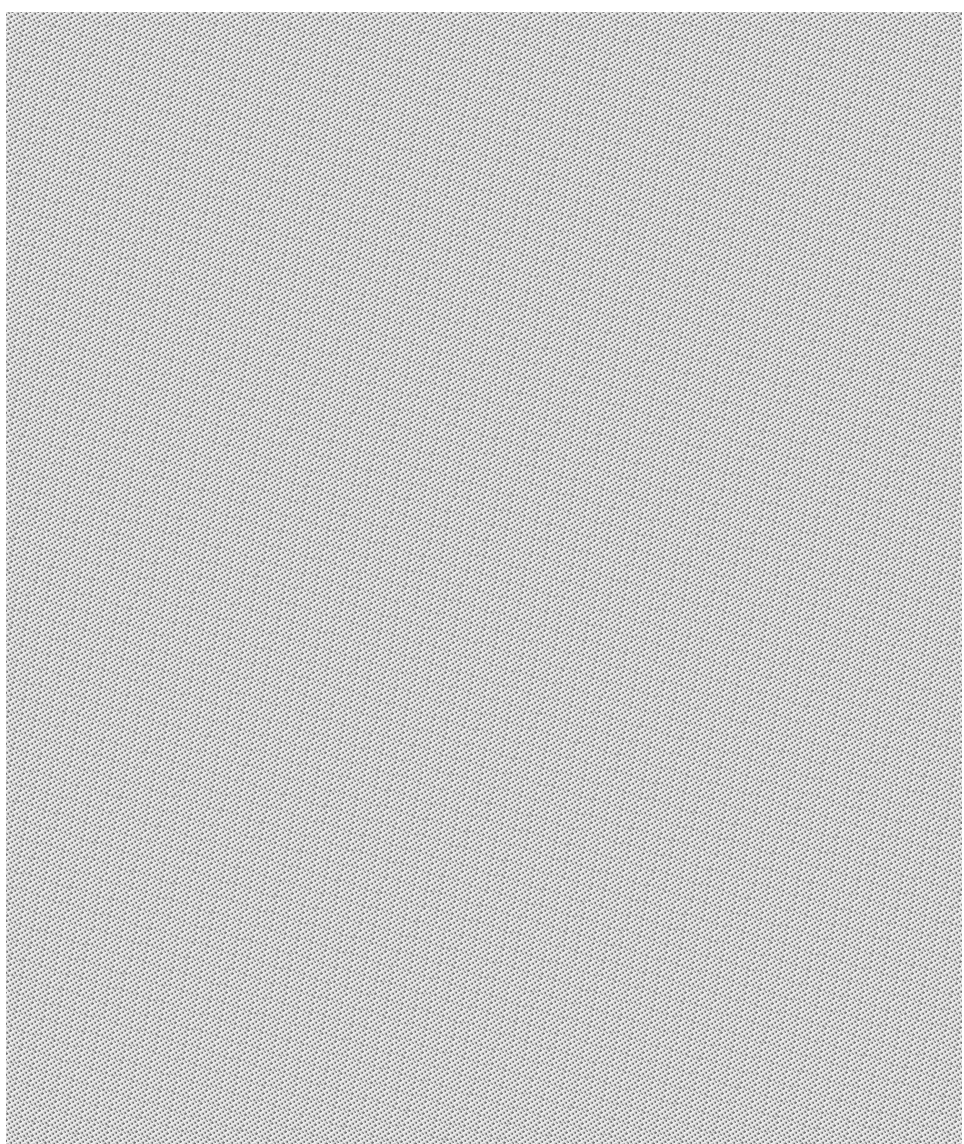

Figure 2.5: The modified X2-based handover procedure to handle MN's movement during a S/PGW relocation in an LTE network with decentralized architecture.

We considered it as a baseline and defined a new handover mechanism for an LTE network with decentralized architecture to cope with the issue described in (I). We modified the Create Session Request/Response and Modify Bearer Request/Response messages between the MME and target S/PGW (which is

\footnotetext{
${ }^{2}$ In the current LTE system, S1-based handover can also be used to handle MNs' mobility during a SGW relocation ( $\S 5.5 .1 .2 .2$ of [20]). As we used the X2-based handover for development of our solutions, in this thesis we ignore to present the required modifications for the S1-based approach.
} 


\subsection{MOBILITY MANAGEMENT IN A DECENTRALIZED LTE NETWORK}

replaced as PGW) to create a new DL-TFT in the target S/PGW. These changes handle the migration of an EPS bearer to a newly established data forwarding plane for the moving MN.

During a X2-based handover procedure with a $\mathrm{S} / \mathrm{PGW}$ relocation, the following messages are exchanged between EPC entities (see Fig. 2.5):

(1) Target eNodeB sends a Path Switch Request message to the MME to inform that MN has changed its cell. This message includes the list of EPS bearers to be switched.

(2) MME stores the IP address, bearers' ID and the TEID used for the source $\mathrm{S} / \mathrm{PGW}$. This information is used to signal the correct $\mathrm{S} / \mathrm{PGW}$ when a bearer needs to be deactivated. Next it sends a Create Session Request message including the bearers context(s) and MN's IP address among other information to the target S/PGW. The procedures of this step are the same as Step (2) in $\S 5.5 .1 .1 .3$ of [20] by replacing the PGW to the target $\mathrm{S} / \mathrm{PGW}$ and removing the procedures related to the $\mathrm{S} 5 / \mathrm{S} 8$ interface.

(3) MME sends a Modify Bearer Request message consisting the target eNodeB's IP address and the TEID to the target S/PGW.

(4) The target S/PGW executes a PCEF Initiated IP-CAN Session Modification procedure with the PCRF to report the new IP Connectivity Access Network (IP-CAN) type. The MN's IP address provided to the PCRF should be the same as received by the MME in the EPS bearer context(s), included in the Create Session Request message [23].

(5) Target S/PGW creates a new entry in its EPS bearer table, among other required information [23], and generates a charging ID for it. The new entry allowing it to route MN's data plane between the target eNodeB and data network. Next, it responds to the MME by a Modify Bearer Response message.

(6) Target S/PGW sends a Create Session Response message back to the MME to inform its IP address(s) and the TEID(s). The MME starts a timer to be used in step (9).

(7) and (8) are the same as steps (5) and (6) in $\S 5.5 .1 .1 .3$ of [20] by replacing the SGW to the S/PGW. 
(9) By ending the timer started in step (6), the MME deletes the EPS bearers (the ones accepted in the target S/PGW) by sending Delete Session Request message to the source S/PGW.

(10) Source S/PGW acknowledges the MME with Delete Session Response message.

(11) Tracking Area Update procedure is the same as steps (9) in $\S$ 5.5.1.1.3 of $[20]$.

More information about the messages and procedures described in this section can be found in $\S 5.5 .1$ of $[20]$.

To realize the function described in (II), we modified the PDN disconnection procedure, specified in $\S 5.10 .3$ of [20]. This procedure allows the MN (or MME) to request for disconnection from the network. During this procedure, the bearers used for the moving $\mathrm{MN}$ shall be removed and the MN's PDN connection (and IP address) can be released. To accomplish these, the following messages are exchanged between EPC entities (see Fig. 2.6):

(1) MN sends a PDN Disconnection Request message, including Linked EPS Bearer ID (LBI), to the MME and asks to disconnect from the network. The LBI indicates the ID of the bearers to be disconnected.

(2) MME sends a Delete Session Request message to the target S/PGW to notify the list of bearers to be released for a particular PDN connection.

(3) Target S/PGW performs an IP-CAN Session Termination procedure $[23]$ to inform the PCRF that the IP-CAN session is released.

(4) Target S/PGW acknowledges the MME with Delete Session Response.

(5) to (9) The related procedures for these steps are the same as the ones defined in $\S 5.10 .3$ of [20].

(10) MME looks up the EPS bearer IDs, if an entry is found as the one indicated in the LBI, it sends a Delete Session Request message to the corresponding $\mathrm{S} / \mathrm{PGW}$.

(11) S/PGW drops the corresponding bearer context from its list and releases the associated MN's IP address(es). Next, it acknowledges the MME 
with Delete Session Response message to remove the corresponding bearers related information.

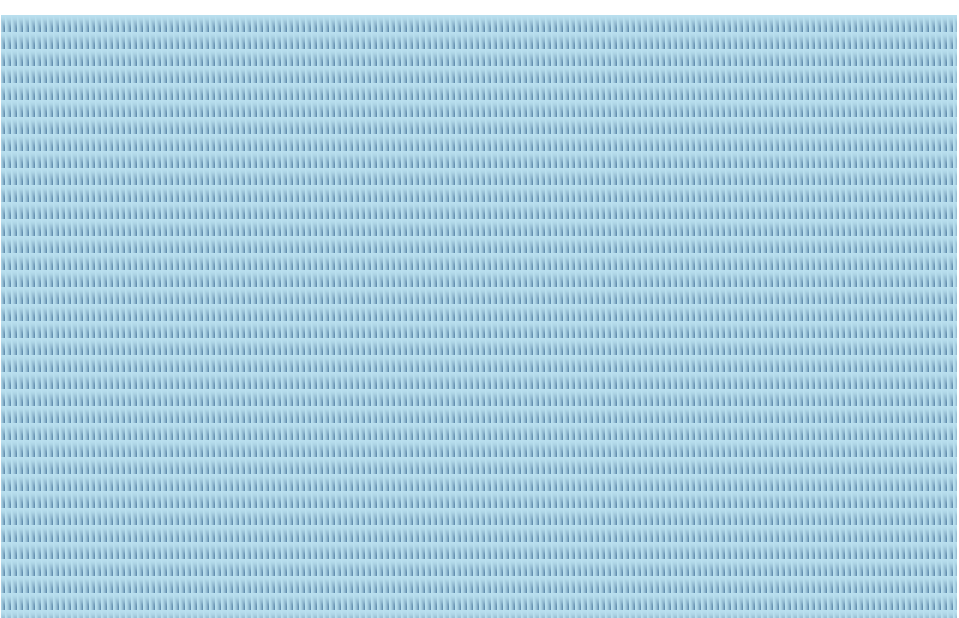

Figure 2.6: The modified PDN disconnection procedure to release MN's bearers and IP address in the corresponding S/PGW(s) in the LTE network with decentralized architecture.

We extend the NS3-LENA simulation environment to implement the LTE network with distributed S/PGWs as well as the modified X2-based handover and PDN disconnection procedures to fulfill the functions described in (I) and (II). The source code for this implementation can be accessed via the link provided in appendix $B$.

\subsection{Summary}

In the current 3GPP's LTE specification, IP-based traffic session continuity is not supported when a MN changes its EPS traffic anchor point (PGW). This is due to the fact that, in the current LTE technical specifications, there is not a standard mechanism to support a handover procedure with PGW relocation and to provide the continuity of bearers after PGW relocation.

Following a decentralized architecture in LTE system, in which the PGW and SGW functionalities combined into a single entity (S/PGW), MNs are expected to change their anchor points $(\mathrm{S} / \mathrm{PGWs})$ far more often. 
The standard control and data planes in the current LTE system specified for the X2-based handover procedure with SGW relocation, can be modified and be applied to support MN's mobility and handover between different S/PGW's domains in the LTE network with decentralized architecture. For this, the required modifications to be accomplished in the current EPC entities and procedures are summarized as follows:

- MN's IP address needs to be stored inside the MME. This address is sent to the MME by source S/PGW during the E-UTRAN Initial Attach procedure using Create Session Response message ( $\S 5.3 .2 .1$ of [20]).

- Target S/PGW need to be informed about the MN's IP address of the EPS bearer, kept active by the MN after handover. This information is sent by the MME using Create Session Request message during the MN's handover procedure.

- PCEF Initiated IP-CAN Session Modification procedure needs to be triggered by the target S/PGW upon reception of Modify Bearer Request message from MME. The MN's IP address received from the MME is used by the $\mathrm{PCEF}$ in this procedure.

- After completion the handover procedure, MME needs to inform the source S/PGW (using Delete Session Request message) to remove the specific EPS bearer, belonging to the moving MN, from its Transferred EPS Bearers list.

- MME needs to inform the source S/PGW to release the moving MN's IP address after PDN disconnection procedure. For this, MME signals the source S/PGW using Delete Session Request message, upon reception of Deactivate EPS Bearer Context Accept message ( $§ 5.10 .3$ of [20]) from target eNodeB. 



\section{Quantitative Comparison of the Centralized and Decentralized LTE Network Architectures}

Although, decentralization of the network architecture would be a promising approach to reduce the traffic load on core network entities, the gain of this approach on the efficiency of LTE network was an open question. Therefore, in this chapter we conduct a hybrid study combining simulation and analytical modeling, aiming to quantify and analyze the impact of the number of connected MNs to the network, on the performance and scalability of the LTE system with centralized and decentralized network architectures. The publication related to this chapter is [72].

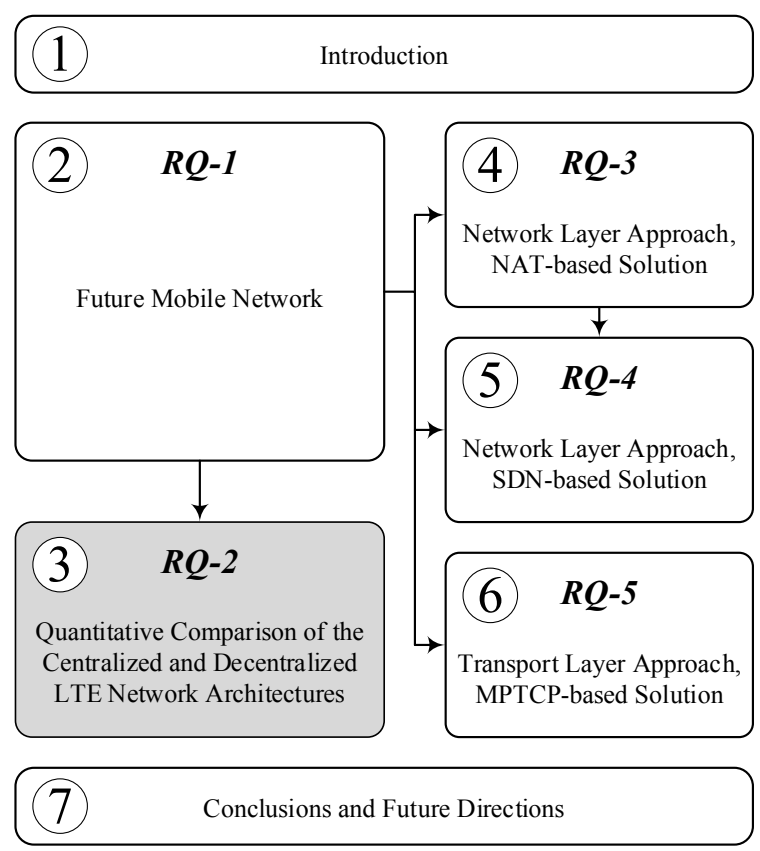


This chapter is organized as follows:

- Section 3.1 reviews in brief the recent related works and specifies how our work differs from the literature.

- Section 3.2 provides concisely the necessary background about the current LTE's data and control planes for 3GPP access.

- Section 3.3 describes the commonly being used protocols in the LTE network to control MN's mobility in 3GPP access network.

- Section 3.4 discuses our hybrid modeling study and details its components.

- Section 3.5 describes the analytical calculation of the performance metrics and the evaluation procedure.

- Section 3.6 presents the obtained results aiming to compare the performance and scalability of the LTE network with centralized and decentralized architectures.

- Section 3.7 concludes this chapter. 


\subsection{Related Work}

To cope with the limitations of current mobile networks architecture e.g., scalability, non-optimal routes, centralized control and data planes several approaches relying on the concept of network structure decentralization, have been proposed and analyzed using simulation and/or modeling. In this section, we reviews in brief the recent related works in this context and state how our work differs from the previous studies.

Using a simulation model, a comparative study has been performed in [30], to analyze performances of the proposed Dynamic Mobility Anchoring (DMA) scheme and MIP protocol on handling the MNs' TCP-based traffic. In this study, the handover latency and TCP segment delays have been used as the performance metrics. The work in [45] presents an analytical and experimental evaluation of PMIP-based mobility management in centralized and distributed ways. The metrics of signaling and packet delivery costs, handover latency, and packet loss have been used to present the trade-offs between two architectures. In a similar way, [62] investigated impact of the distribution and dynamic activation of the mobility anchors on performance of the PMIP protocol. In this study, the evaluation has been accomplished based on the packet delivery cost, anchored/non-anchored packet ratio, and traffic distribution ratio. The authors in [103] proposed a PMIPv6-based Distributed mobility management model (DPMIPv6), which outperformed the conventional PMIPv6 in terms of route optimization, packet delivery and signaling costs. The discussed approach relied on distributing of access routers with a centralized management model. A comprehensive study of Distributed and Dynamic Mobility Management (DDMM) has been done by [63]. In this work, the authors discussed an architecture with distributed deployment of mobility anchors with dynamic activation. In this research, it was shown that DDMM generally achieves higher performance compared to centralized mobility management in terms of packet delivery cost, tunneling overhead, and throughput. The work in [79] proposed a Partially Distributed Mobility Management (P-DMM) and a Fully Distributed Mobility Management (F-DMM). In the former approach only the data plane was distributed, while in the latter one both data and control planes were distributed. In this work, it was shown that the F-DMM outperforms the P-DMM in terms of handover latency and packet loss. In [43], the functional differences of the GTP and PMIP, within the EPC have been discussed and the signalling costs of these protocols, using dynamic QoS and policy control, have been evaluated. An introduction of the different LTE core network architectures and mobility management schemes have been presented in [99]. In this work, an analytical model based on the two-dimensional hexagonal random walk model was proposed for comparing performance of the mobility management on different EPC 
core architectures. For the performance evaluations the total signalling cost and load on the network nodes have been used as the comparison parameters.

Compared to previous studies, that mostly focused on the performance analysis of the MIP/PMIP protocols in general centralized network as well as the proposed distributed mobility management approaches, we paid special attention to the LTE network. We choose the LTE system for our study as currently it is the dominant mobile networking technology and also is expected to accommodate the major part of worldwide mobile data traffic in coming decade. Our work differs from the literature, particularly with respect to comprehensive analysis of four main possible scenarios on the LTE system i.e., the current (centralized) and a decentralized EPC architectures with GTP or PMIP mobility protocols for $3 \mathrm{GPP}$ access.

We conduct a hybrid study comprising simulation and analytical models to compare and analyze performance of current and decentralized LTE network architectures. The proposed model enables detailed analysis of impact of the number of attached mobile nodes on the load at network routers and links, on the latency, and on the processing cost of the user's data and control planes. We investigate performance of GTP and PMIP, the commonly being used protocols in the LTE, to handle mobile nodes data traffic and mobility in 3GPP access. To the best of our knowledge, this is the first work to perform a quantitative analysis of such scale on the LTE system in order to compare the performance of different network architectures.

\subsection{LTE's Data and Control Planes}

This section gives a brief overview on the necessary background about the current LTE's data and control planes for 3GPP access. These are essential information to be used in the next sections on modeling and studying the performance of the GTP and PMIP protocols in the LTE network, being performed in this chapter.

When a MN attaches to the LTE network, several control messages are exchanged between the eNodeB, MME, SGW, and PGW. Using GTP protocol, a successful attachment results in a tunnel for the user data plane (GTP-U) between the eNodeB and SGW, and between the SGW and PGW. Another tunnel for the control plane (GTP-C) is established between the SGW and PGW and also between the MME and SGW, see Fig. 3.1. While GTP-U simply transports data packets within the core and radio access networks, GTP-C tunnel is used to exchange the control messages for handling MN's mobility, as well as for path management and tunnel management (e.g., to adjust QoS parameters, 


\section{Quantitative Comparison of the Centralized and Decentralized LTE 38 Network Architectures}

to update sessions for the roaming MNs, and to activate and deactivate MNs' sessions).

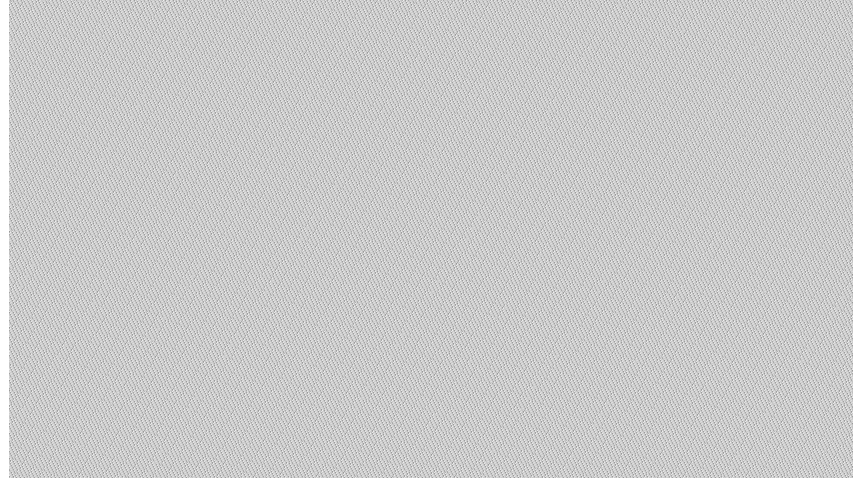

Figure 3.1: GTP and PMIP protocols headers.

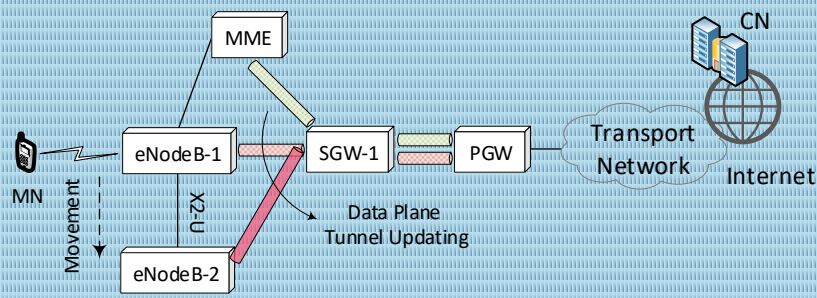

(a) MN's handover without SGW relocation

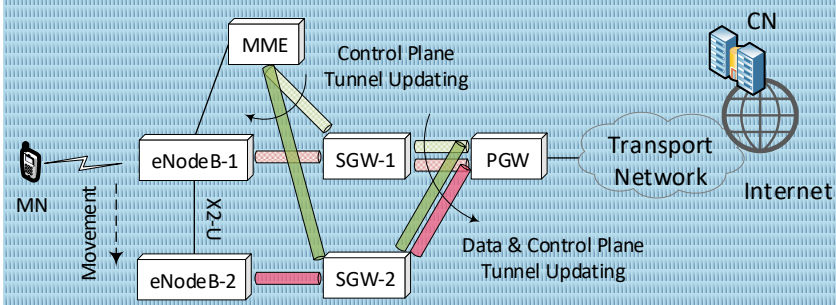

(b) MN's handover with SGW relocation

$\square$ Control Plane Tunnel $\square \quad$ Data Plane Tunnel

Figure 3.2: Tunnel updating during MN's handover without (a) and with (b) SGW relocation. 
These tunnels are instantiated for each individual MN's traffic flow (IP traffic). Each GTP tunnel uses an unique identifier (TEID). Based on the TEID, the network is able to choose the appropriate tunnels to transfer data packets and control messages between the end points.

In the case of PMIP protocol, basic IP connectivity over GRE tunneling is used between the SGW and PGW, and a GRE key is used to identify each tunnel.

As Fig. 3.2 shows, during a handover between neighboring eNodeBs the GTP-U tunnel is updated, and if the handover is happen between neighboring SGWs both GTP-U and GTP-C (or GRE) are updated. Note that tunneled packets might be further encapsulated by IPsec protocol in order to protect both control messages and data. In this study we only focus on the data and control planes' tunnels established over the transport network. Additional control signaling involved on MN's attachment, handover and data delivery procedures with the other LTE components as well as IPsec protocol are out of the scope in this thesis.

\subsection{LTE's Mobility Management Messages}

The signaling for MN's mobility control is similar for the GTP and PMIP protocols in the access network. Differences depend on the mobility protocol applied in the core network over the S5/S8 interfaces, see Fig. 2.1.

Following sections, describe in brief the GTP and PMIP protocols' messages, exchanged between the EPC's entities during MN's attachment and handover procedures.

\subsubsection{For the GTP Protocol}

For GTP protocol, when MN's switch is turned on, an Attach Request message is sent to the MME through the eNodeB. The MME then sends a Create Session Request message to the SGW, which is forwarded to the PGW. This request is meant for setting up the MN's default bearer and also for requesting a PDN connectivity. The reply from the PGW (Create Session Response), containing an IP address for the MN and a default bearer ID, is also forwarded by the SGW to the eNodeB and the MME, see Fig. 3.3. With this information the attachment procedure is concluded and the traffic from the MN can flow from the eNodeB to the SGW via the S1-U interface (Fig. 3.1). The MME still sends a Modify Bearer Request message to the SGW, which in turn is forwarded to the PGW, containing the TEID assigned to the eNodeB. The PGW finally replies to this request with a Modify Bearer Response message, and the user data plane is then set up for traffic flow in the core network. 


\section{Quantitative Comparison of the Centralized and Decentralized LTE 40 Network Architectures}

During a handover between eNodeBs, the target eNodeB sends a Path Switch Request to the MME, informing that the MN has changed its physical location. The MME then sends to the SGW a Modify Bearer Request message with the address of the new eNodeB and the TEID of the user plane for downlink. This information is forwarded by the SGW to the PGW. The PGW replies with a Modify Bearer Response message to the SGW, that starts forwarding downlink packets to the target eNodeB (current MN location).

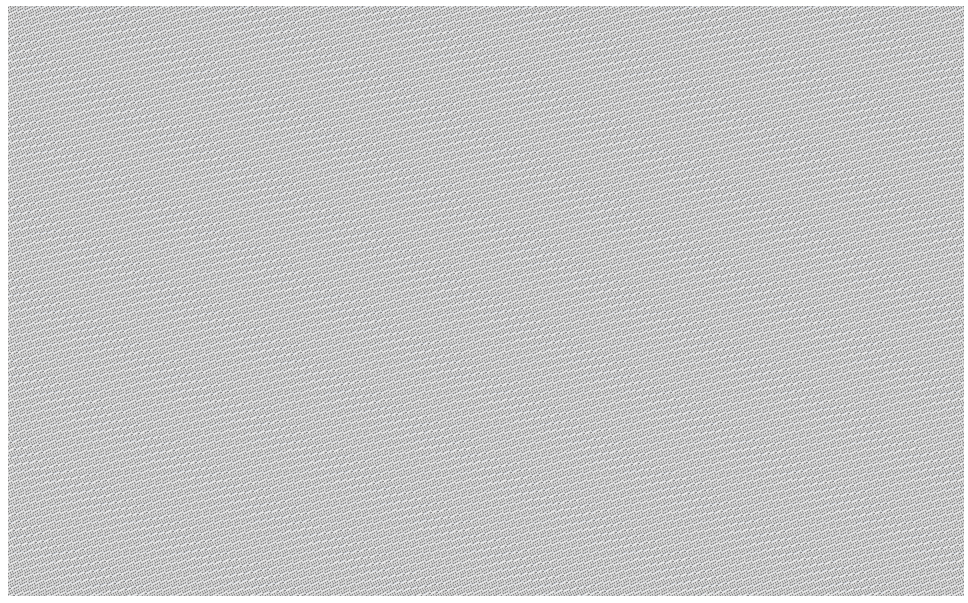

Figure 3.3: GTP protocol messages during MN's attachment and handover procedures.

Table 3.1: Size of the GTP protocol mobility control messages [89].

\begin{tabular}{|l|l|l|}
\hline Message & Size & Headers \\
\hline \hline Create Session Request (C.S.Req) & $335 \mathrm{~B}$ & $60 \mathrm{~B}$ \\
Create Session Response (C.S.Res) & $224 \mathrm{~B}$ & $60 \mathrm{~B}$ \\
Modify Bearer Request (M.B.Req) & $67 \mathrm{~B}$ & $60 \mathrm{~B}$ \\
Modify Bearer Response (M.B.Res) & $81 \mathrm{~B}$ & $60 \mathrm{~B}$ \\
\hline
\end{tabular}

On realizing that the SGW has been relocated (from the Path Switch Request message sent by the target eNodeB), the MME sends a Create Session Request message to the target SGW. This message contains the PGW addresses, the TEIDs used for uplink traffic, the address of the target eNodeB, and the protocol 
type used over S5/S8 interface. The target SGW assigns the addresses and TEIDs for downlink traffic from the PGW, and sends a Modify Bearer Request message to the PGW informing about the changes. The PGW then updates its context field and replies to the SGW with a Modify Bearer Response message including its address and TEIDs information [20].

Table 3.1 lists size of the GTP protocol messages used during MN's attachment and handover procedures. Note that the size information does not include the extra GTP-C tunnel header size, which is presented in a separated column.

\subsubsection{For the PMIP Protocol}

For PMIP the initial attachment procedure of a MN is similar to that of the GTP. The only difference is that the SGW has to establish a control session towards the PCRF to obtain the QoS policy information needed to perform the bearer binding. All these are obtained through a Gateway Control Session Response message sent by the PCRF to the SGW, in reply to a Gateway Control Session Request message. This messages exchange is done over the Stream Control Transmission Protocol (SCTP), see Fig. 3.4.

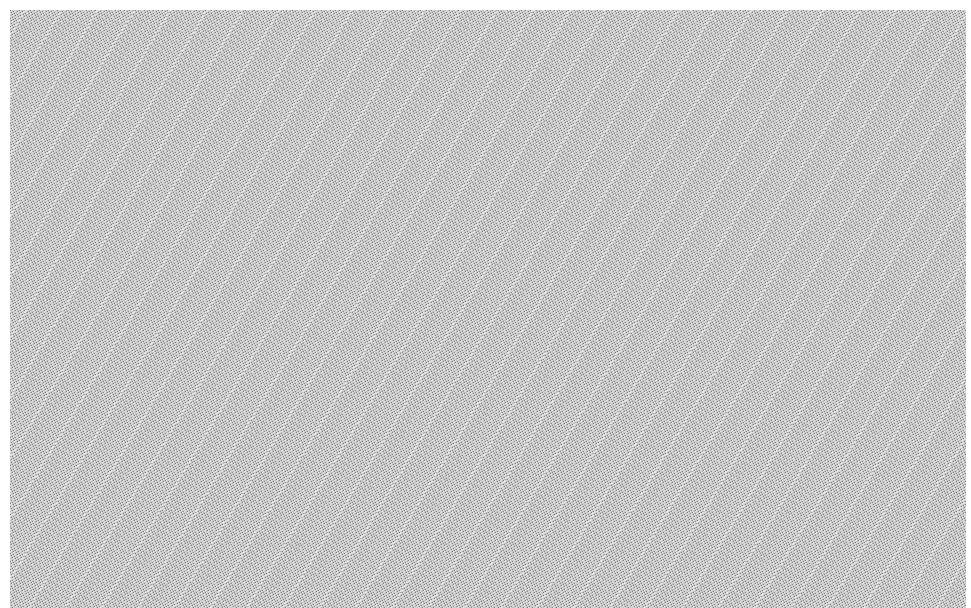

Figure 3.4: PMIP protocol messages during MN's attachment and handover procedures.

To establish the default bearers, the SGW sends to the PGW a Proxy Binding Update message containing, among others, the GRE key for downlink traffic, information of the MN to request an IP address, and charging characteristics. The PGW replies to the SGW with a Proxy Binding Acknowledge message 


\section{Quantitative Comparison of the Centralized and Decentralized LTE 42 Network Architectures}

containing, among others, the MN's IP address, the GRE key for uplink traffic, and the charging ID. These two messages are exchanged over a GRE tunnel, and after this exchange the SGW and the PGW set up an additional bi-directional GRE tunnel for forwarding of MN's data flows.

During handover between eNodeBs, the SGW sends to the PCRF a Gateway Control Session Request message informing about the change of MN's location, which was received from the MME. The PCRF replies to the SGW with a Gateway Control Session Response message providing, among others, the updated QoS policy and charging rules. In case of a handover with SGW relocation, in addition to the PCRF messages, the new SGW have to exchange the Proxy Binding Update and Proxy Binding Acknowledge messages with the PGW [21].

Table 3.2: Size of the PMIP protocol mobility control messages [43].

\begin{tabular}{|l|l|l|}
\hline Message & Size & Headers \\
\hline \hline Gateway Control Session Request (G.C.S.Req) & $336 \mathrm{~B}$ & $68 \mathrm{~B}$ \\
Gateway Control Session Response (G.C.S.Res) & $972 \mathrm{~B}$ & $68 \mathrm{~B}$ \\
Proxy Binding Update (P.B.U) & $104 \mathrm{~B}$ & $64 \mathrm{~B}$ \\
Proxy Binding Acknowledge (P.B.A) & $104 \mathrm{~B}$ & $64 \mathrm{~B}$ \\
\hline
\end{tabular}

Table 3.2 lists the messages used in the procedures explained above. Note that the size information does not include additional header sizes, which can differ and are presented in a separated column.

In the following, $\S 3.4$ describes our hybrid modeling approach to quantify the gains on performance and scalability in four different scenarios; the centralized (current) and a decentralized (future) EPC architecture with GTP and PMIP mobility management protocols. Next in $\S 3.5$, we present the calculation of various performance metrics in detail.

\subsection{Hybrid Simulation and Analytical Modeling}

This section describes our hybrid study containing simulation and analytical modelings. The simulation model captures the dynamic behaviour of the system at $\mathrm{MN}$ and connection level, delivering information about e.g., network links and nodes load. This information, together with other network and traffic parameters is used in the analytical delay model to derive queuing delay for data packets and control messages processing in the nodes as well as queuing delays for transmitting these information on the network links. 


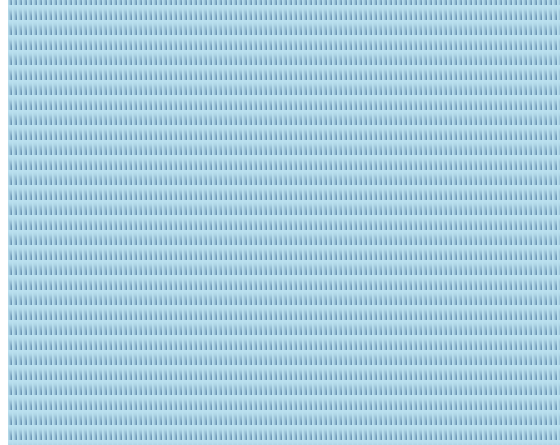

Figure 3.5: The hybrid modeling study components.

Table 3.3: The notations used in the hybrid model and analytical calculation.

\begin{tabular}{|c|c|c|}
\hline Symbol & Definition & Value \\
\hline$R$ & Radius of each SGW (S/PGW) & \\
\hline$A$ & Coverage area of each SGW (S/PGW) & \\
\hline$P$ & Perimeter of each SGW (S/PGW) & \\
\hline$H_{x-y}$ & Number of hops between arbitrary nodes $x$ and $y$ & \\
\hline$d_{(x)_{l}}$ & Queuing delay of $x$ in the network link $l$, due to traffic load & $x \in\left\{p,\left(p+t_{u}\right),\left(m+t_{c}\right)\right\}$ \\
\hline$\delta_{(x)_{l}}$ & Transmission delay of $x$ in the network link $l$ & $x \in\left\{p,\left(p+t_{u}\right),\left(m+t_{c}\right)\right\}$ \\
\hline$\tau_{p_{(p / m)}}$ & Packet $(p)$ or Message $(m)$ processing delay in router $j$ & \\
\hline$\tau_{r_{(p / m)}}$ & $p$ or $m$ routing delay in router $j$ & \\
\hline$\tau_{t}(p / m)_{x}$ & $p$ or $m$ tunneling delay in the node $x$ & $x \in\{S G W, P G W, S / P G W\}$ \\
\hline$d_{(p / m)_{j}}$ & $p$ or $m$ queuing delay in router $j$ due to processing load & \\
\hline$N_{h_{i}}$ & Number of handovers $(h)$ for node $i$ during simulation time & \\
\hline$N_{k_{i}}$ & Number of paths $(k)$ created for node $i$ due to mobility & \\
\hline$M N A P_{(x)}$ & Messages Number of the Initial Attachment Procedure & $x \in\{G T P, P M I P\}$ \\
\hline$M N H P_{(x)}$ & Messages Number of the Handover Procedure & $x \in\{G T P, P M I P\}$ \\
\hline$A L D P D_{i}$ & Average Latency of Packet Delivery for node $i$ & \\
\hline$A C D P D_{i}$ & Average processing Cost of Packet Delivery for node $i$ & \\
\hline$L D P D_{k}$ & Latency of Packet Delivery in path $k$ & \\
\hline$C D P D_{k}$ & Processing Cost of Packet Delivery in path $k$ & \\
\hline$L A P_{i}$ & Latency of Attachment Procedure for node $i$ & \\
\hline$C A P_{i}$ & Processing Cost of Attachment Procedure for node $i$ & \\
\hline$L H P_{i}$ & Latency of Handover Procedure for node $i$ & \\
\hline$C H P_{i}$ & Processing Cost of Handover Procedure for node $i$ & \\
\hline$C M L_{(N)}$ & Message Lode (number) over router $j$ during simulation time & \\
\hline$C M L_{(K B s)}$ & Message Load (size) over router $j$ during simulation time & \\
\hline
\end{tabular}




\section{Quantitative Comparison of the Centralized and Decentralized LTE 44 Network Architectures}

Eventually, these intermediate results are used to calculate end-to-end data packet delivery delay and cost, control plane and data plane loads. Fig. 3.5 shows schematically how the various parts of our hybrid model relate to each other.

It is important to remark that, in our approach, on one hand we capture the most essential characteristics of the system, and on the other hand abstract from the less important details to set up an straightforward environment to perform the modelings and analyses in a feasible time. We use MATLAB as the environment to implement the models and perform the analysis. Using a normal desktop computer, it takes only a couple of minutes to carry out the complete tasks for all scenarios.

Table 3.3 presents the notation used in the hybrid model as well as in the analytical calculation to compute different performance metrics, see $\S 3.5$.

The link of the source code for implementation of all the components of our hybrid modeling study, and for the detailed calculation of the performance metrics can be in appendix B.

\subsubsection{Network Topology Model}

Fig. 3.6 shows the network topology used in our modeling. This topology follows the Cisco 3-layer hierarchical model consisting of the core, distribution and access layers. The core layer handles traffic transferred to and from the routers at the distribution layer. The distribution layer enables the communication between routers from the core and access layers. The access layer mainly controls the attachment of end users and mobile devices to the network.

We define two network scenarios in our models: the current (centralized) and future (decentralized) network architectures in context of the LTE system. In each of these, the routers in the core and the access layers assume different roles.

In the first scenario, for the centralized architecture, the PGW is placed in the top layer of the topology ( $R 1$ in Fig. 3.6), and the SGWs are placed at the edge network ( $R 6$ to $R 11$ ). In this scenario the PGW is the anchoring point of the network and handles all data and control plane operations. The SGWs provide the data paths towards the core, and route MN's packets between the access network and the PGW.

In the second scenario, for the decentralized architecture, we define S/PGWs, which are physical nodes combining functions of the SGW and PGW elements. In this scenario the S/PGWs operate as the distributed anchoring points and handle the data and control planes operations locally. These are placed in the access layer ( $R 6$ to $R 11)$. Herein, the core router $(R 1)$ is only used when data has to be routed through it. 
We consider three static CNs located at each layer. These represent the data centers that in reality could be geographically distributed. The data traffic towards the MNs is transmitted with the shortest path through $(\mathrm{CN} \rightarrow \mathrm{PGW} \rightarrow \mathrm{SGW} \rightarrow \mathrm{MN})$ or $(\mathrm{CN} \rightarrow \mathrm{S} / \mathrm{PGW} \rightarrow \mathrm{MN})$ for the centralized and decentralized scenarios, respectively. It is important to mention that we ignore all detailed functions of the routing mechanism (e.g., load balancing) in our model. As in this chapter we perform a comparative analysis for the different network architectures, using a plain routing solution has no severe effect on the final outcome of the comparison.

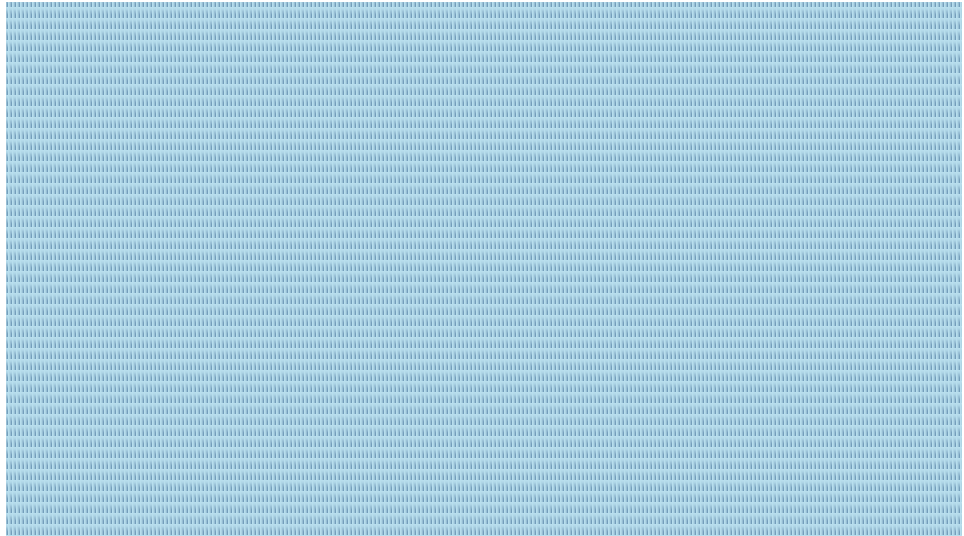

\begin{tabular}{|l|c|c|c|}
\cline { 2 - 4 } \multicolumn{1}{c|}{} & R1 function & R2-R5 function & R6-R1 function \\
\hline Centralized approach & PGW & Router & SGW \\
\hline Decentralized approach & Router & Router & S/PGW \\
\hline
\end{tabular}

Figure 3.6: The network topology used in the analytical model.

The two network architecture scenarios described above, are later (in $\S 3.5$ ) combined with both the GTP and PMIP protocols to define the four scenarios, being analyzed in this chapter.

\subsubsection{Mobility Model}

A straightforward approach to model mobility is by obtaining the time spent by a $\mathrm{MN}$ in each $\mathrm{SGW}$ (S/PGW) during movement (also known as the residence time or the dwell time). The Fluid-Flow mobility model is a simple approach to model node mobility in cellular networks, and it has been extensively applied in 
previous work $[88,86,79,45]$. By applying the Fluid-Flow model where $E(v)$ is the average speed of a MN, the average dwell time of the node in each SGW is given by:

$$
E\left[T_{d w e l l}\right]=\frac{\pi \times A}{E(v) \times P}=\frac{\pi \times\left(\pi R^{2}\right)}{E(v) \times(2 \pi R)}=\frac{\pi R}{2 E(v)}
$$

Note that for each MN, $E(v)$ is arbitrarily chosen from the predefined values listed in Table 3.5.

We assume that a mobile node randomly attaches to one of the SGWs and after passing the dwell time it starts to move towards the neighbor SGW. This movement is modeled by randomly choosing one of the neighbor SGWs and staying in it for the dwell time. This procedure is continuously repeated during the simulation time. Therefore, the number of handovers for each mobile node $\left(N_{h_{i}}\right)$, can be easily derived using its dwell time and simulation time. For both scenarios, mobile nodes choose the same trajectories, affecting the paths created for the related control and data planes during the simulation time. In our model we assume that $E(v)$ for each mobile node is constant during the simulation time. It is also assumed that each SGW covers a circular domain consisting of three eNodeBs, and therefore for a SGW relocation there will be three handovers between neighboring eNodeBs.

\subsubsection{Traffic Model}

We assume that each MN randomly attaches to one of the SGWs (or S/PGWs) and downloads data from one of the CNs, also randomly chosen. Every MN has a single active session to one of the CNs during the whole simulation time. Every node follows the mobility model described in the previous section and after staying at each access node for its dwell time moves to one of the neighboring access nodes.

A Poisson traffic stream with average rate of 100 packets per second $(C N T R)$ is generated from the CNs towards the connected nodes, simulating the download of data by the attached nodes. For the sake of simplicity, this model ignore the packet level details (e.g., packet loss and loss recovery mechanism).

To avoid IP packet fragmentation as a result of tunneling overhead at the core network, we set the size of packets from the CNs to 1200 Bytes ([19] advises a default MTU size of 1280 Bytes).

\subsubsection{Delay Model}

During a transmission between two endpoints data packets or control messages may be delayed due to, for example, link congestion and queues. 
In our model the network link delay in each hop includes the transmission delay $\delta_{(x)_{l}}$ and the queuing delay $d_{(x)_{l}}$, where $x$ is either a pure or a tunneled data packet or control message (see Table 3.3). Applying an M/M/1 queuing model, the average delay $T_{(p)_{l}}$ of a data packet of size $p$ transmitted in the network link $l$, with transmission rate $T R$ and traffic load $C N T R$ per $\mathrm{MN}$ is given by:

$$
T_{(p)_{l}}=\left[\delta_{(p)_{l}}+d_{(p)_{l}}\right]=\frac{\delta_{(p)_{l}}}{1-L_{(p)_{l}}} \quad, \quad L_{(p)_{l}}=\frac{\lambda_{l} \times p}{T R}
$$

The $\lambda_{l}$, denotes the network link traffic, derived from the simulation by keeping track of all paths that are established using link $l$, as well as their duration and taking into account the packet rate $C N T R$.

For a data packet $p$ or a control message $m$, and a node $j$, the network node delay consists of the processing delay $\tau_{p_{(p / m)}}$, the data packet encapsulation/decapsulation or control message construction/extraction delay $\left(\tau_{t(p / m)_{j}}\right)$ during a tunneling, the routing delay $\tau_{r_{(p / m)}}$, and the queuing delay $d_{(p / m)_{j}}$ at the node (see Table 3.3). For matters of simplicity we assume that $\tau_{p_{(p / m)_{j}}}=\tau_{t_{(p / m)}}=\tau_{r_{(p / m)}}$.

Similar to the network link delay, for a network node $j$ with the processing rate $P R$, the average delay $T_{(p)_{j}}$ for a data packet $p$ is defined by:

$$
T_{(p)_{j}}=\left[\tau_{p_{(p)_{j}}}+d_{(p)_{j}}\right]=\frac{\tau_{p_{(p)_{j}}}}{1-L_{(p)_{j}}} \quad, \quad L_{(p)_{j}}=\frac{\lambda_{j} \times p}{P R}
$$

Herein, $\lambda_{j}$ signifies the network node traffic, obtained from the simulation by keeping track of all paths that are crossed router $j$, as well as their duration.

\subsection{Calculation of the Performance Metrics}

This section presents the analytical calculation of the performance metrics (see Table 3.4), defined in our model to quantify the impact of the number of MNs on the performance of the EPC with current and decentralized network architectures, using the GTP and PMIP protocols.

In current EPC architecture, PGW handles centrally the MNs' data traffic and mobility though the whole network. However, in decentralized architecture, the S/PGWs being distributed closer to edge of the network, manage traffic of the MNs attached locally and handle their mobility when moving between the eNodeBs. Therefore, an additional mechanism needs to be implemented on top of the S/PGWs to keep ongoing traffic sessions active for the MNs performing 


\section{Quantitative Comparison of the Centralized and Decentralized LTE 48 Network Architectures}

handovers with S/PGW relocation. Different approaches may demand additional components and modifications in the network topology as well as impose further signaling efforts in the network, which must also be taken into account, see $[34,69,71,70,46]$ as examples.

In our model we only study the parameters related to the core network. That is because the structures and characteristics for the access and wireless networks are the same for both the centralized and decentralized LTE architectures.

Table 3.4: Performance Metrics.

\begin{tabular}{|l|l|}
\hline Symbol & Definition \\
\hline \hline$A L D P D$ & Average Latency of Data Packet Delivery \\
$A C D P D$ & Average processing Cost of Data Packet Delivery \\
$A L A P$ & Average Latency of initial Attachment Procedure \\
$C A P$ & Processing Cost of initial Attachment Procedure \\
$A L H P$ & Average Latency of Handover Procedure \\
$A C H P$ & Average processing Cost of Handover Procedure \\
$L R$ & Load of Routers \\
$L N L$ & Load of Network Links \\
$C M L_{(N)}$ & Control Messages Load (number) \\
$C M L_{(K B s)}$ & Control Messages Load (size) \\
\hline
\end{tabular}

In the following, $\S$ 3.5.1 defines the performance metrics listed in Table 3.4. Next, we detail the latency and processing cost related metrics for both architectures in $\S 3.5 .2$ and 3.5.3, respectively.

\subsubsection{Definition of Performance Metrics}

- Average Latency of Data Packet Delivery (ALDPD)

The $A L D P D$ is obtained using the average latency of data packet delivery for each mobile node $i$ :

$$
A L D P D=\frac{\sum_{i=1}^{N_{\text {node }}} A L D P D_{i}}{N_{\text {node }}}
$$

The individual ALDPD for a mobile node $i$ is given by:

$$
A L D P D_{i}=L A P_{i}+\overline{L H P_{i}}+\overline{L D P D_{i}}
$$

where

$$
\overline{L H P_{i}}=\frac{\sum_{h_{i}=1}^{N_{h_{i}}} L H P_{h_{i}}}{N_{h_{i}}} \text { and } \overline{L D P D_{i}}=\frac{\sum_{k_{i}=1}^{N_{k_{i}}} L D P D_{k_{i}}}{N_{k_{i}}}
$$


Note that $L A P_{i}$ is the latency of the initial attachment procedure for mobile node $i$. The $\overline{L H P_{i}}$ and $\overline{L D P D_{i}}$ define the average handover latency and the average latency for data packet delivery over the created paths (due to mobility) for mobile node $i$, respectively.

- Average Processing Cost of Data Packet Delivery (ACDPD)

The $A C D P D$ is obtained by:

$$
A C D P D=\sum_{i=1}^{N_{\text {node }}} A C D P D_{i}
$$

The individual $A C D P D$ for a mobile node $i$ is given by:

$$
A C D P D_{i}=C A P_{i}+\overline{C H P_{i}}+\overline{C D P D_{i}}
$$

where

$$
\overline{C H P_{i}}=\frac{\sum_{h_{i}=1}^{N_{h_{i}} C H P_{h_{i}}}}{N_{h_{i}}} \text { and } \overline{C D P D_{i}}=\frac{\sum_{k_{i}=1}^{N_{k_{i}}} C D P D_{k_{i}}}{N_{k_{i}}}
$$

Herein, $C A P_{i}$ is the processing cost of the initial attachment procedure for the mobile node $i$. The $\overline{C H P_{i}}$ and $\overline{C D P D_{i}}$, define the average handover processing cost and the average processing cost for data packet delivery over the created paths (due to mobility) for mobile node $i$, respectively.

One may note that $L A P_{i}$ and $C A P_{i}$ may slightly affect the overall amount of the $A L D P D$ and $A C D P D$, respectively. However, we would like to discuss all parameters involved during the data packet delivery procedure to acquire more precise results.

- Average Latency of initial Attachment Procedure (ALAP)

It is given by:

$$
A L A P=\frac{\sum_{i=1}^{N_{\text {node }}} L A P_{i}}{N_{\text {node }}}
$$

- Processing Cost of initial Attachment Procedure (CAP)

The $C A P$ is obtained by:

$$
C A P=\sum_{i=1}^{N_{\text {node }}} C A P_{i}
$$

- Average Latency for Handover Procedure (ALHP) 
The $A L H P$ is given by:

$$
A L H P=\frac{\sum_{i=1}^{N_{\text {node }}} \overline{L H P_{i}}}{N_{\text {node }}}
$$

- Average Processing Cost for Handover Procedure (ACHP)

It is given by:

$$
A C H P=\sum_{i=1}^{N_{\text {node }}} \overline{C H P_{i}}
$$

- Load of the network Routers (LR) and Load of the Network Links (LNL)

The $L R$ and $L N L$ define the data plane loads, implying that how many times MNs traffic pass through the network routers and links, respectively.

- Control Message Load (CML) in terms of number $\left(C M L_{(N)}\right)$ and size of messages $\left(C M L_{(K B s)}\right)$

The $C M L_{(N)}$ defines the load of control plane at the network routers in terms of the number of messages.

It is obtained by:

$$
C M L_{(N)_{j}}=\sum_{i=1}^{N_{\text {node }}}\left(M N A P_{(G T P / P M I P)}+M N H P_{(G T P / P M I P)} \times N_{h_{i}}\right)
$$

We count the $C M L_{(N)}$ for both EPC network architectures using the GTP and PMIP protocols.

The $C M L_{(K B s)}$ is another interpretation of $C M L_{(N)}$, that takes into account also the size of messages and represents a better view of control plane related load at the network routers. Considering that the control messages type and size for the GTP and PMIP protocols are different, making the expression of $C M L_{(K B s)}$ more complex, we avoid to present it here.

In the following, we elaborate on the $A L D P D_{i}$ and $A C D P D_{i}$, covering also the latency of the initial attachment $\left(L A P_{i}\right)$ and the average handover $\left(\overline{L H P_{i}}\right)$ procedures as well as the related costs $\left(C A P_{i}\right.$ and $\left.\overline{C H P_{i}}\right)$, respectively. Abbreviation of the control messages used in the following sections are listed in Table 3.1 and 3.2. Moreover, the notations for the elements used in the analytical calculation of the performance metrics as well as the input parameters are listed in Table 3.3 and Table 3.5 and 3.6, respectively. 


\subsection{2 $A L D P D$ for individual mobile node}

$A L D P D_{i}$ from $\mathrm{Eq} .3 .5$, consists of the latency of the initial attachment procedure $\left(L A P_{i}\right)$, the average latency of handover procedure $\left(\overline{L H P_{i}}\right)$, and the mean latency of data packet delivery $\left(\overline{L D P D_{i}}\right)$ for node $i$, in the paths created from $\mathrm{CN}$ to the access layer routers.

In following we detail these items for the centralized and decentralized LTE architectures, considering both GTP and PMIP protocols.

\subsubsection{The Centralized Architecture}

\section{- The GTP-based approach}

Referring to Fig. 3.3, $L A P_{i}$ defines the latency of MN initial attachment procedure, caused by exchanging Create Session Request/Response and Modify Bearer Request/Response messages between the attached SGW and PGW. That is the delay of tunneling (constructing/extracting) of the messages in the attached SGW and PGW, and also the delay of routing those messages (over the GTPC) in the path between them.

$\overline{L H P_{i}}$ is calculated using the latency of handover in each path $\left(L H P_{h_{i}}\right)$ during the simulation time. For both eNodeB and SGW relocations, Modify Bearer Request/Response messages are exchanged between the attached SGW and PGW. Therefore, $L H P_{h_{i}}$ includes: (i) the delay of tunneling of messages at the attached SGW (for eNodeB relocation) or at the second SGW (for SGW relocation) and $\mathrm{PGW}$; and (ii) the delay of routing the tunneled messages in the path built between them.

\section{- The PMIP-based approach}

In the PMIP-based approach the initial attachment latency includes: (i) the delay for exchanging Gateway Control Session Request/Response messages (over SCTP protocol) between the attached SGW and PCRF; (ii) the delay for tunneling the Proxy Binding Update/Acknowledge messages at the attached SGW and PGW; and (iii) the delay of routing the messages (over the GRE) in the created path (Fig. 3.4).

$L H P_{h_{i}}$ defines the delay for exchanging Gateway Control Session Request/Response messages (over the SCTP protocol) between the first SGW (for eNodeB relocation) or the second SGW (for SGW relocation) and PCRF. In case of SGW relocation, it also includes the delay of tunneling of Proxy Binding Update/Acknowledge messages in the second SGW and PGW, and the delay of routing the messages in the path between them. 


\section{Quantitative Comparison of the Centralized and Decentralized LTE 52 Network Architectures}

Finally, $L D P D_{k_{i}}$ specifies the delay of data packet delivery from $\mathrm{CN}$ to SGW in path $k$. In both GTP and PMIP approaches the $L D P D_{k_{i}}$ includes: (i) the delay of routing IP packets between CN and PGW; (ii) the delay of data packet encapsulation (over GTP-U or GRE) on PGW; (iii) the delay of routing the tunneled packets in the path between PGW and SGW; and (iv) the delay of data packet decapsulation in SGW.

If the MN experiences a handover with SGW relocation during it's session time, data packets have to be tunneled between two SGWs. The delay caused by this procedure must also be taken in to account in $L D P D_{k_{i}}$.

The detailed derivation of $A L D P D_{i}$ for the centralized architecture is given in appendix A.1.1.

\subsubsection{The Decentralized Architecture}

In the decentralized architecture, S/PGW performs both SGW and PGW functionalities. The control messages are not tunneled during the initial attachment and handover procedures, and neither the data packets are forwarded between PGW and SGW. The only GTP tunneling is between S/PGW and eNodeBs, which is also the case for the centralized architecture (Fig. 3.7).

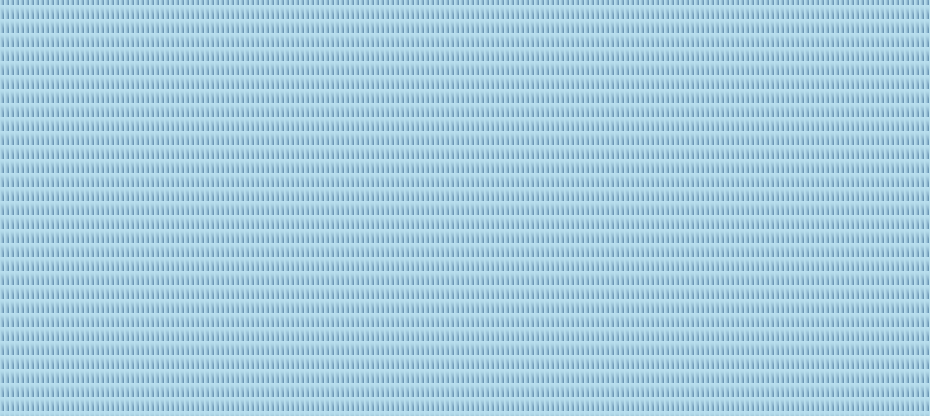

Figure 3.7: Date and control planes tunnel updating during MN's handover (with $\mathrm{S} / \mathrm{PGW}$ relocation) in a decentralized LTE system.

\section{- The GTP-based approach}

$L A P_{i}$ only includes the delay of processing the Create Session Request/Response and Modify Bearer Request/Response messages on S/PGW. Similarly, the delay of $\mathrm{LHP}_{h}$ is for processing the Modify Bearer Request/Response messages in the 
first attached S/PGW during eNodeB relocation, and in the second one for $\mathrm{S} / \mathrm{PGW}$ relocation.

\section{- The PMIP-based approach}

The delay of $L A P_{i}$ is for processing the Gateway Control Session Request/Response, including the SCTP and IPv6 protocol headers, and Proxy Binding Update/Acknowledge messages on S/PGW. $L H P_{h_{i}}$ for eNodeB relocation only includes the processing delay of Gateway Control Session Request/Response messages in $\mathrm{S} / \mathrm{PGW}$. In the case of $\mathrm{S} / \mathrm{PGW}$ relocation the delay is due to processing of the Gateway Control Session Request/Response and Proxy Binding Update/Acknowledge messages in target S/PGW.

In the decentralized architecture, regular IP data packets with no tunneling are forwarded through CNs to S/PGWs. Therefore, $L D P D_{k_{i}}$ only includes the delay for routing the packets in the path between an arbitrary CN and S/PGW.

Similar to the centralized architecture in the case of a S/PGW relocation during node session time the delay for forwarding the tunneled data packet between two S/PGW must be considered.

The detailed derivation of $A L D P D_{i}$ for the decentralized architecture is given in appendix A.1.2.

\subsection{3 $A C D P D$ for individual mobile node}

$A C D P D_{i}$ from $\mathrm{Eq} .3 .7$, includes the cost of handling mobility control messages $\left(C A P_{i}\right.$ and $\left.C H P_{h_{i}}\right)$, and the cost of data packet delivery $\left(C D P D_{k_{i}}\right)$ from CN to the access layer entities in the created paths, during the MN's session time. This section describes the parameters for both centralized and decentralized approaches and details these parameters for GTP and PMIP protocols. For the sake of simplicity, we assume that the costs of the processing $\left(C_{p}\right)$, routing $\left(C_{r}\right)$, and tunneling $\left(C_{t}\right)$ in the routers are the same. Furthermore, given the traffic load crossing through the routers at each layer, we assign $1,1 / 3,1 / 4$ unit processing cost per each $K B$ of traffic for the root router (PGW in centralized architecture), the distribution routers, and the access routers (SGWs in centralized architecture and S/PGW in decentralized architecture), respectively. This is a rational comparative assignment for the purpose of comparing network architectures.

\subsubsection{The Centralized Architecture}

\section{- The GTP-based approach}




\section{Quantitative Comparison of the Centralized and Decentralized LTE 54 Network Architectures}

In the GTP-based approach, $C A P_{i}$ defines the cost of exchanging Create Session Request/Response and Modify Bearer Request/Response tunneled messages between the first attached SGW and PGW. It also includes the cost of constructing/extracting of messages in the first SGW and PGW, and the cost of routing the tunneled messages (over GTP-C) in the path between them.

Similarly, $\mathrm{CHP}_{h_{i}}$, describes the cost of transferring only the Modify Bearer Request/Response tunneled messages (over GTP-C) between the first attached SGW and PGW during eNodeB relocation, or the target SGW and PGW for SGW relocation.

\section{- The PMIP-based approach}

For the PMIP protocol, $C A P_{i}$ comprises: (i) the costs of swapping the Gateway Control Session Request/Response messages, including the SCTP and IPv6 protocol headers, between the first attached SGW and PCRF; (ii) the cost of tunneling (constructing/extracting) the Proxy Binding Update/Acknowledge messages in the first attached SGW and PGW; and (iii) the cost of routing the tunneled messages over GRE in the path between them.

$C H P_{h_{i}}$, for eNodeB relocation only includes the cost of processing Gateway Control Session Request/Response messages exchanged between the first SGW and PCRF. During SGW relocation, $C H P_{h_{i}}$ defines the cost of exchanging aforesaid messages between the target SGW and PCRF. Moreover, it includes the cost of exchanging the tunneled Proxy Binding Update/Acknowledge messages over GRE between the second SGW and PGW.

$C D P D_{k_{i}}$ specifies the cost of routing regular IP packets from CN to PGW, and the cost of tunneling (encapsulating) data packets over GTP-U or GRE in PGW. It also counts for the cost of routing the tunneled packet in the path between PGW and SGW, and also the cost of decapsulating the tunneled packet in SGW.

The cost of forwarding the tunneled data packets between two SGWs must also be considered in case of a SGW realocation during the session time.

The detailed derivation of $A C D P D_{i}$ for the centralized architecture is given in appendix A.2.1.

\subsubsection{The Decentralized Architecture}

\section{- The GTP-based approach}

As described in $\S 3.5 .2 .2$, the decentralized architecture does not have a control or data plane tunneling in the core network. Therefore, $C A P_{i}$ only includes the cost of processing Create Session Request/Response and Modify Bearer Request/Response messages in the $\mathrm{S} / \mathrm{PGW}$. 
$\mathrm{CHP}_{h_{i}}$ includes the cost of processing Modify Bearer Request/Response messages in the first attached $\mathrm{S} / \mathrm{PGW}$ for eNodeB relocation, and in the target $\mathrm{S} / \mathrm{PGW}$ during $\mathrm{S} / \mathrm{PGW}$ relocation.

\section{- The PMIP-based approach}

In this approach, $C A P_{i}$ is related to the cost of processing Gateway Control Session Request/Response messages, including the SCTP and IPv6 protocol headers, and the Proxy Binding Update/Acknowledge messages in S/PGW.

$\mathrm{CHP}_{h_{i}}$, for eNodeB relocation only defines the cost of processing the Gateway Control Session Request/Response messages, including the SCTP and IPv6 protocol headers, in the first S/PGW. During S/PGW relocation it describes the cost of processing the Gateway Control Session Request/Response and Proxy Binding Update/Acknowledge messages in the second S/PGW.

In decentralized architecture, $C D P D_{k_{i}}$ specifies the cost of routing IP packets without tunneling header in the path between $\mathrm{CN}$ and S/PGW. If a MN experiences handover during the session time, $C D P D_{k_{i}}$ also includes the cost of forwarding the tunneled data packets between the first and target S/GWs.

Note that, in both network architectures, if there is more than one SGW (or S/PGW) between $\mathrm{CN}$ and MN the data packets are tunneled and forwarded among them. Therefore, the additional delay and cost due to this procedure also must be considered in calculating $L D P D_{k_{i}}$ and $C D P D_{k_{i}}$.

The detailed derivation of $A C D P D_{i}$ for the decentralized architecture is given in appendix A.2.2. 


\section{Quantitative Comparison of the Centralized and Decentralized LTE 56 Network Architectures}

\subsection{Numerical Results}

This section presents the numerical results of the performance metrics, defined in $\S$ 3.5. The obtained results provide scalability indicators for the EPC centralized and decentralized network architectures via a quantitative analogy over the performance of GTP and PMIP protocols. Table 3.5 lists the input parameters and Table 3.6 summarizes the default parameters used in the $\S 3.4$ and $\S 3.5$.

Table 3.5: Input Parameters (Assumptions).

\begin{tabular}{|l|l|l|}
\hline Symbol & Definition & Value \\
\hline \hline$N_{n o d e}$ & Number of mobile nodes & {$[100 \ldots 1000]$} \\
$E(v)$ & Average velocity of mobile nodes & 25,50 or $75 \mathrm{~km} / \mathrm{h}$ \\
$R$ & Radius of the coverage area of the access routers & $1200 \mathrm{~m}$ \\
$p$ & Average data packet size & 1200 Bytes \\
$C N T R$ & CNs generating traffic (a poisson traffic stream) & With average 100 pps \\
$P R$ & Routers processing rate & $1000 \mathrm{~K}$ pps \\
$T R$ & Network links transmission rate & $100 \mathrm{~K}$ pps \\
$C_{P G W}$ & Cost of processing $C_{p}$, routing $C_{r}$, or tunneling $C_{t}$ at PGW & 1 unit per KB \\
$C_{S G W}$ & Cost of processing $C_{p}$, routing $C_{r}$, or tunneling $C_{t}$ at SGW & $1 / 3$ unit per KB \\
$C_{r_{j}}$ & Cost of routing at the distribution router $j$ & $1 / 4$ unit per KB \\
\hline
\end{tabular}

Table 3.6: Standard parameters (§ 3.2 and $\S 3.3)$.

\begin{tabular}{|l|l|l|}
\hline Symbol & Definition & Size \\
\hline \hline$t_{u_{(G T P)}}$ & GTP-U header & $64 B$ \\
$t_{c_{(G T P)}}$ & GTP-C header & $60 B$ \\
if $m=$ C.S.Req & GTP Create Session Request message & $335 B$ \\
if $m=$ C.S.Res & GTP Create Session Response message & $224 B$ \\
if $m=$ M.B.Req & GTP Modify Bearer Request message & $67 B$ \\
if $m=$ M.B.Res & GTP Modify Bearer Response message & $81 B$ \\
$t_{(P M I P v 6)}$ & PMIPv6 tunnel header & $64 B$ \\
if $m=G . C . S . R e q$ & PMIPv6 Gateway Control Session Request message & $336 B$ \\
if $m=G . C . S . R e s$ & PMIPv6 Gateway Control Session Response message & $972 B$ \\
if $m=P . B . U$ & PMIPv6 Proxy Binding Update message & $104 B$ \\
if $m=P . B . A$ & PMIPv6 Proxy Binding Acknowledge message & $104 B$ \\
\hline
\end{tabular}

As a means of providing reliable performance results and hence insight into the system behavior, we provide mean results with $95 \%$ confidence interval by repeating the simulations for the same setting, using 10 random seeds. Note 
that due to partial overlap of the confidence intervals for different input values, they are presented for limited number of measurement points and graphs, for better presentation purpose.

\subsubsection{Average cost and latency of MN's initial attach- ment, handover, and data packet delivery proce- dures.}

The graphs in Fig. 3.8 show the impact of the number of MNs on GTP (solid lines) and PMIP (dash lines) performance in terms of cost and latency, for both the EPC centralized (red lines) and decentralized (blue lines) architectures. It is notable that the decentralized architecture outperforms the centralized one, regardless of the mobility protocol. This is because in the decentralized architecture the control plane messages without tunneling are handled in the S/PGWs of access layer. Furthermore, data traffic with regular IP packets are only transmitted over the paths between CNs and S/PGWs without crossing the root node. Accordingly, latency and cost measures are substantially improved.

\section{- The MN's initial attachment procedure}

Fig. $3.8 \mathrm{a}$ and $3.8 \mathrm{~b}$ show that in centralized architecture during the initial attachment procedure, PMIP achieves better outcomes than GTP particularly in latency. This is due to the fact that four messages in GTP and two messages in PMIP are exchanged (over GTP-C and GRE, respectively) between the SGWs and PGW. The other two messages in PMIP are exchanged between the SGW and PCRF, through a private network link without tunneling (see Fig. 3.4). In decentralized architecture, the $\mathrm{S} / \mathrm{PGW}$ handle these messages. Hence, the related latency and cost are only due to processing of messages on the S/PGWs, having no tunneling header. In this scenario, GTP provides better results as its initial attachment messages are smaller than PMIP messages.

For the centralized architecture, Fig. 3.8a shows that the growth rate of the attachment latency in PMIP is $5 \%$ less than GTP. However, in the decentralized architecture GTP performs $4 \%$ better than PMIP. In addition, using decentralized architecture, the attachment latency is improved $\approx 44$ and $\approx 8$ times in GTP and PMIP, respectively (see Table 3.7).

Fig. 3.8b shows that for attachment cost in centralized architecture PMIP provides $(>1 \%)$ lower increasing slope compared to GTP. However, in the decentralized architecture this metric for GTP is $\approx 7 \%$ lower than for PMIP. Furthermore, in decentralized architecture GTP and PMIP decrease the attachment cost by $\approx 9$ and $\approx 3$ times, respectively, compared to the centralized architecture (see Table 3.7). 


\section{Quantitative Comparison of the Centralized and Decentralized LTE

\section{- The handover procedure}

Fig. 3.8c shows that in centralized architecture PMIP offers a lower handover latency than GTP. This is caused by the latency of routing two tunneled messages between the attached SGW and PGW in GTP for every eNodeB relocation. For PMIP the messages are only processed and exchanged between the SGW and PCRP, via a dedicated link. For the cost metric, GTP outperforms PMIP (see Fig. 3.8d). Because in GTP both the control plane tunnel headers and handover messages are smaller than PMIP. Furthermore, GTP uses fewer number of messages during SGW relocation. In the decentralized architecture, GTP shows better functionality than PMIP both in terms of latency and cost (see Fig. 3.8c and $3.8 \mathrm{~d}$ ). This is due to the processing of fewer short-sized handover messages in GTP on S/PGWs. Fig. 3.8c indicates that for centralized architecture PMIP shows $2 \%$ lower growth rate on handover latency than GTP. In decentralized architecture, GTP outperforms PMIP and provides $7 \%$ lower growth rate for this metric.

Moreover, the handover latency is also improved by $\approx 62$ and $\approx 3$ times in GTP and PMIP, respectively. In terms of handover cost, for both architectures GTP carries out better than PMIP, achieving $2.6 \%$ and $12.7 \%$ lower increasing slope for this metric in the centralized and decentralized architectures, respectively (see Table 3.7). Furthermore, for decentralized architecture GTP reduces the handover cost by $\approx 12$ times compared with centralized one, which this is $\approx 2$ times when PMIP is used (see Fig. 3.8d).

\section{- The data packet delivery procedure}

As shown in Fig. 3.1, size of the tunnels header in both GTP and PMIP data planes are the same, and hence, one may expect similar latency and cost for both protocols. However, recalling from Eq .3.4 and Eq .3.6, these metrics also depend on the latency and cost on the attachment and handover procedures, resulting in the similar outcomes.

Fig. 3.8e shows that PMIP performs $2 \%$ better than GTP for centralized architecture in terms of growth rate for latency on data packet delivery. However, in decentralized architecture GTP outperforms PMIP and provides 1\% lower growth rate for this metrics. Furthermore, GTP improves latency for $\approx 11 \%$ times, which is $\approx 6 \%$ far from PMIP.

For the cost metric, GTP provides better results than PMIP with $\approx 1 \%$ and $\approx 9 \%$ lower increasing slope in the centralized and decentralized architectures. In addition, for decentralized architecture GTP reduces the data packet delivery cost by $\approx 6$ times compared to centralized one, while PMIP achieves a ratio of $\approx 3$ times (see Fig. 3.8f). 


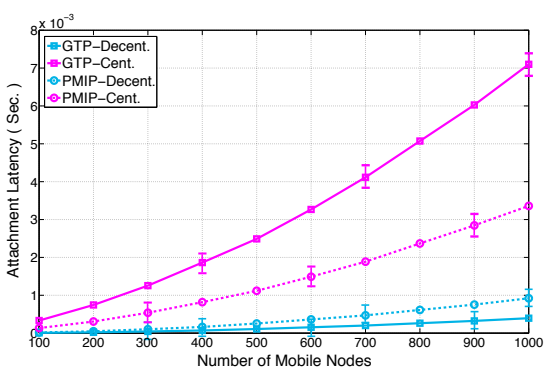

(a) Latency of initial attachment procedure.

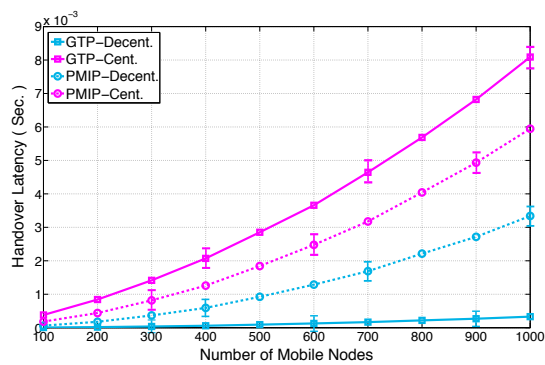

(c) Latency of handover procedure.

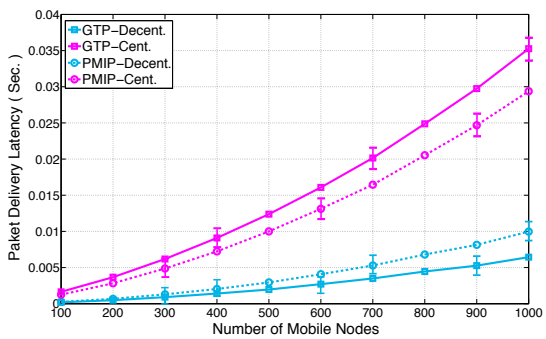

(e) Latency of packet delivery procedure.

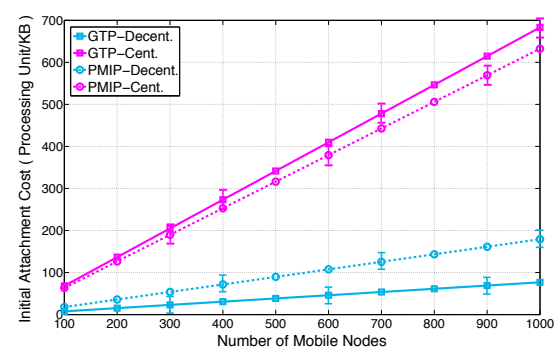

(b) Cost of initial attachment procedure.

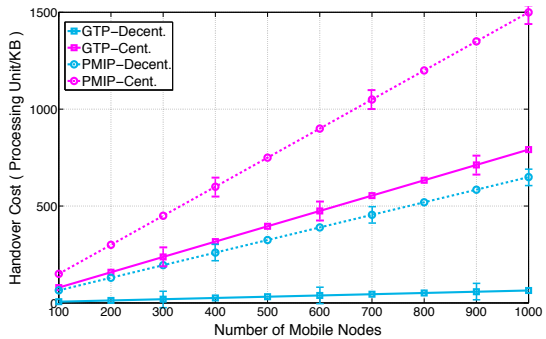

(d) Cost of handover procedure.

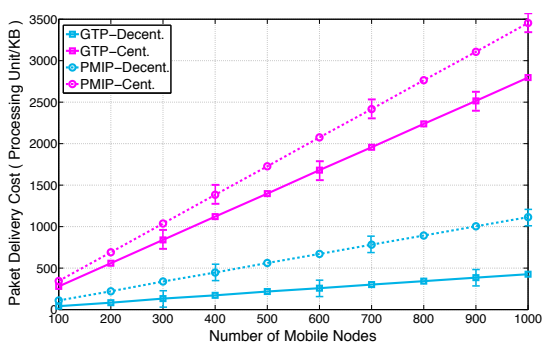

(f) Cost of packet delivery procedure.

Figure 3.8: The latency and cost of GTP and PMIP mobility protocols in EPC centralized and decentralized architectures. 


\section{Quantitative Comparison of the Centralized and Decentralized LTE}

Table 3.7, summarizes the results discussed in this section. In this table, the percentile values represent the Growth Rate (GR) (of exponentially growing parameters) or Increasing Slope (IS) (of lineally growing parameters) of the parameters, and the scalar values show the ratio of the initial value of the parameters in centralized to decentralized architecture $\left(Y_{100}\right.$ centralizec $/ Y_{100}$ decentralizec $)$.

Table 3.7: Performance of GTP and PMIP in the various EPC network architectures.

\begin{tabular}{|l|l|l|l|l|l|l|}
\hline & \multicolumn{2}{|l|}{ Centralized } & \multicolumn{2}{l|}{ Decentralized } & \multicolumn{2}{l|}{ Ratio } \\
\hline & GTP & PMIP & GTP & PMIP & GTP & PMIP \\
\hline \hline LAP & $55 \%$ & $50 \%$ & $40 \%$ & $44 \%$ & 44.35 & 7.63 \\
\hline LHP & $55 \%$ & $53 \%$ & $40 \%$ & $47 \%$ & 61.83 & 2.94 \\
\hline LDPD & $52 \%$ & $50 \%$ & $41 \%$ & $42 \%$ & 11.54 & 5.83 \\
\hline CAP & $155.8 \%$ & $154.7 \%$ & $144.1 \%$ & $151.5 \%$ & 8.90 & 3.52 \\
\hline CHP & $153.8 \%$ & $156.4 \%$ & $141.6 \%$ & $154.3 \%$ & 12.31 & 2.30 \\
\hline CDPD & $156.7 \%$ & $157.2 \%$ & $147.3 \%$ & $156.1 \%$ & 6.74 & 3.13 \\
\hline
\end{tabular}

Exponentially growing parameters (the latency metrics, see Fig. 3.8a, 3.8c and 3.8e) in general are denoted by: $Y_{n}=Y_{n_{0}} \times(1+G R)^{n}$;

Linearly growing parameters (the processing cost metrics, see Fig. 3.8b, 3.8d and 3.8f) in general are denoted by: $Y_{n}=Y_{n_{0}}+I S \times n$,

Where $Y_{n}$ represents the performance metric corresponding to $n$ number of MNs and $n_{0}=100$, see Fig. 3.8.

\subsubsection{The data plane and control plane load on the net- work routers and links}

Fig. 3.9 shows the impact of the number of MNs on load of the network routers and links for centralized and decentralized architectures. Although we expected a higher loads of both data and control planes in decentralized architecture than in centralized one, we observe that the differences are surprisingly large. That is because in decentralized architecture the control plane messages as well as the data traffic are managed and handled at anchor points placed in the access layer, resulting in reduced load and stress in the upper layers.

\section{- Load of the data plane}

Fig. $3.9 \mathrm{a}$ and $3.9 \mathrm{~b}$ show load of the data plane on the network routers and links for different EPC network architectures, respectively. 


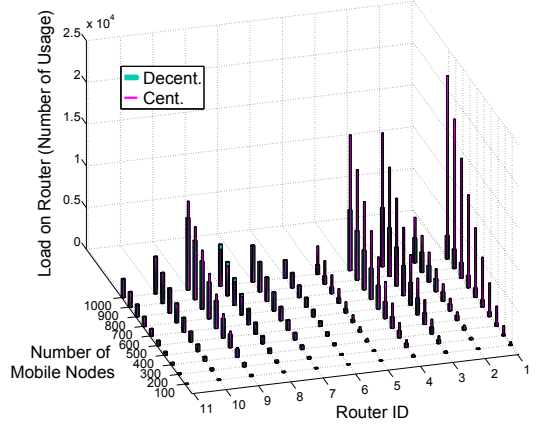

(a) Data plane load on the routers.

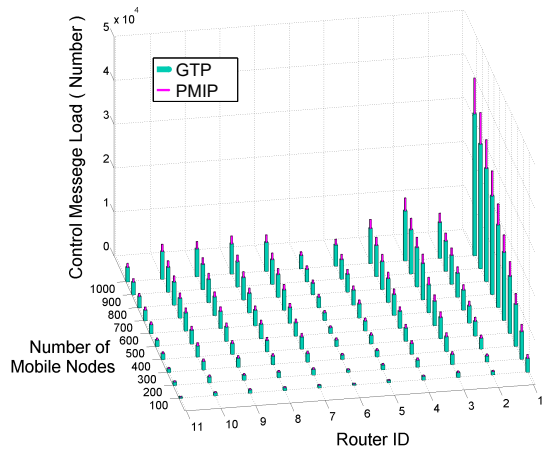

(c) Control messages load (numbers) on the routers in centralized architecture.

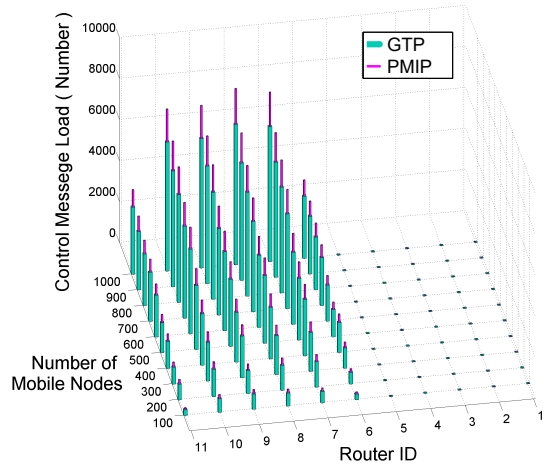

(e) Control messages load (numbers) on the routers in decentralized architecture.

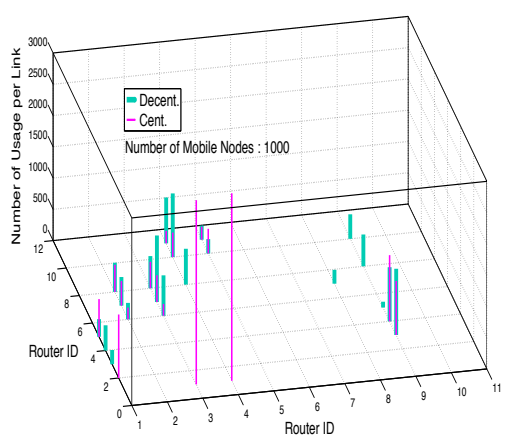

(b) Data plane load on the network links.

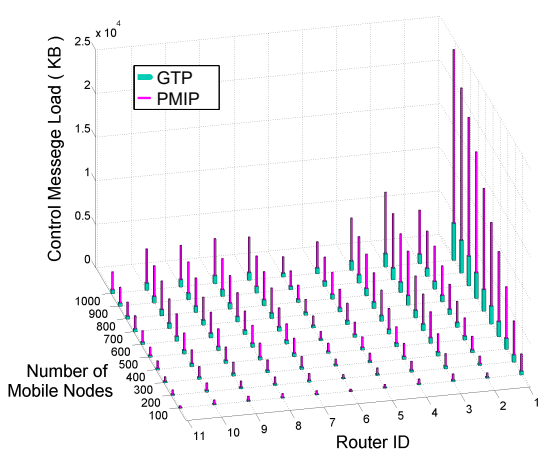

(d) Control messages load (KB) on the routers in centralized architecture.

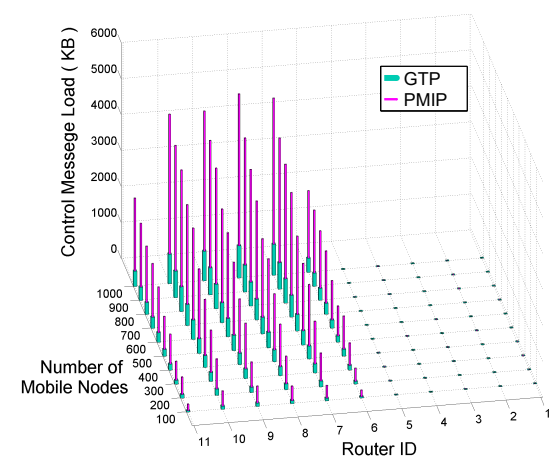

(f) Control messages load (KB) on the routers in decentralized architecture.

Figure 3.9: Load of the data and control planes, over the network routers and links in EPC centralized and decentralized architectures. 
As expected, the root router (R1-PGW in Fig. 3.6) and the routers on the distribution layer $(R 2$ to $R 5)$ in the centralized approach, are used more often than in the decentralized one. Accordingly, the network links between these two layers, forwarding data traffic between the routers are also used more in the centralized approach.

In decentralized architecture MNs' traffic is mainly handled by the access layer entities ( $R 6$ to $R 11-S / P G W$ ) and load is more distributed over the network. This reduces the stress on upper layers and eliminates load of the core routers and links by growing the number of attached MNs.

Note that, in both architectures some of the network links are not used. That is because in the proposed model the path construction between the MNs and CNs is only based on the shortest-paths approach, disregarding other functionalities such as load balancing.

Note also that, in decentralized architecture the load on router $R 9$ is slightly higher than in centralized architecture. This is because in the former, $R 9$ is directly connected to $C N 3$ and directly serves the MNs that link to CN3.

In addition in centralized architecture, the obtained results show that the load of the root router $(R 1)$ is $\approx 11$ times more than in decentralized architecture. For the routers placed in the distribution and access layers this load ratio on average is $\approx 2$ and $\approx 1$ times, respectively.

\section{- Load of the control plane}

Fig. 3.9c and 3.9e show the impact of the number of MNs on loads of GTP and PMIP control messages within the routers for different EPC architectures. In centralized architecture all messages related to MN's mobility are handled by the root router $(\mathrm{PGW})$. Therefore, the routers at the distribution layer $(R 2$ to $R 5$ ) have also to be involved on crossing the messages within the core network. However, in decentralized architecture control messages are not traversed to the upper layer routers and the access layer routers (S/PGWs in our model) are in charge of managing the mobility messages.

Fig. $3.9 \mathrm{~d}$ and $3.9 \mathrm{f}$ show loads on the network routers due to the control plane messages in terms of amount (KBs) of the handling messages. It is observed that PMIP protocol impacts more load to the network than GTP protocol. This is because size of the mobility messages and the tunneling headers for PMIP are larger than for GTP. Furthermore, GTP generates less amount of control messages during the SGW or S/PGW relocation (see Fig. 3.1 and 3.3).

Our results indicate that for both GTP and PMIP protocols, the control plane load on the routers is amplified linearly by the number of attached nodes to the network. It is also observed that PMIP imposes in average $\approx 6$ times higher load on the routers than GTP. 


\subsection{Conclusions}

Although, decentralization of the core network architecture is not standardized by the $3 \mathrm{GPP}$ yet, it is seen as the vision on emerging future mobile network (e.g., $5 \mathrm{G})$ architectural standards. In this regard there are different ongoing research projects and activities, aiming to come up with the solutions to address the features and demands of current mobile networks in a decentralized architecture. In this chapter, we have particularly conducted a detailed analysis and comparative study of centralized and decentralized network architectures in the LTE system for $3 \mathrm{GPP}$ access. We have carried out a hybrid study comprising simulation and analytical modeling to evaluate the attached node's (device's) data traffic and mobility related messages load, as well as the latency and cost for the initial attachment, handover, and data packet delivery procedures in both network architectures, using GTP and PMIP protocols. Our research aimed, in particular, to quantify the impact of the number of connected devices to the network, on various performance and scalability metrics for both LTE network architectures.

Given the specified scenarios and parameters in our study, the optioned results show that decentralization of the LTE network architecture substantially reduces load of data traffic $(\approx 11$ times) on the core of the network. Accordingly, this leads to improvement of the latency for attachment $(\approx 44$ times $)$ and handover $(\approx 61$ times) procedures during the node mobility, and the latency for data delivery procedure $(\approx 11$ times) (using GTP). These are the keys parameters in providing higher QoS and QoE for the subscribers. It is also shown that a decentralized architecture (using GTP) imposes remarkably lower processing cost on both the data plane $(\approx 7$ times $)$ and control plane $(\approx 11$ times during handover), (see Table 3.7, which is also an essential concern for network operators. The analysis indicated that, in the centralized architecture, PMIP achieves slightly lower growing rates for the latencies but provides higher increasing slopes for the loads and costs compared to GTP protocol. However, in a decentralized architecture, GTP protocol outperforms PMIP for all the performance metrics.

The outcomes of this research provide an initial insight on the impact of the growing number of users on the efficiency and scalability of the current and decentralized LTE architectures. As future study, our analysis can be further extended by taking into account other parameters e.g., the additional control plane overhead, needed to carry out the core network features (e.g., traffic monitoring, policy controlling), the required investment and the level of complexity for modification the network architectures. Ultimately, this can provide the mobile network operators a trustworthy intuition on decision making and policy 
Quantitative Comparison of the Centralized and Decentralized LTE 64

Network Architectures

development procedures to accommodate the network for coping with the future mobile data traffic. 



\section{Network Layer Approach, NAT-based Solution}

In this chapter we describe a network layer-based mobility support approach relying on Network Address Translation (NAT), which is widely used in the current Internet. The proposed solution can easily handle traffic steering and session continuity, above the EPC network (see § 2.3), for the MNs' moving among the $S / P G W$ s in a decentralized LTE architecture. This chapter details the architecture, function and components of the proposed solution, and presents the performance metrics and the obtained results of the evaluation. The publications related to this chapter are [68, 69].

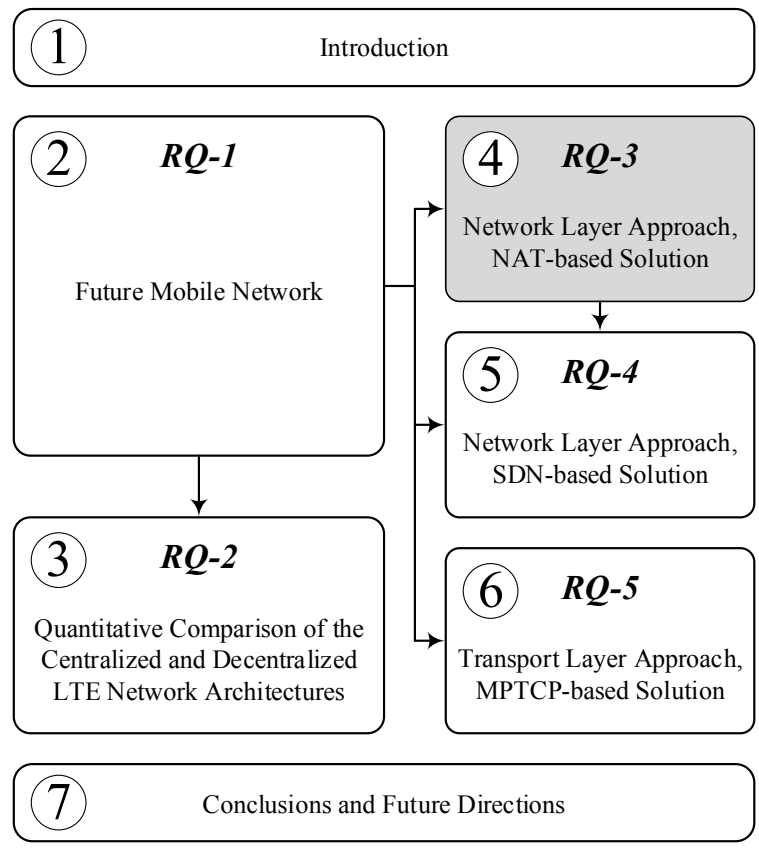


This chapter is organized as follows:

- Section 4.1 reviews briefly recent related work and states the contribution of this chapter.

- Section 4.2 describes the functional approach of the proposed solution.

- Section 4.3 presents the architecture, components, and control messages of the proposed solution.

- Section 4.4 describes the evaluation scenario and implementation of its components in the NS3-LENA simulation environment.

- Section 4.5 defines setting of the parameters in the simulation environment.

- Section 4.6 defines the performance metrics for the validation and presents and discusses the obtained results accordingly.

- Section 4.7 concludes this chapter. 


\subsection{Related Work}

In this section we briefly look at the recent related works and state how our work differs from the literature.

In the data networks, IP address is used in the network layer protocols to locate a node in the network topology to steer traffic packets towards it. The same IP address is also used in the transport and application layers to identify the communication session. Hence, when the node moves to a new network domain and obtains a new IP address, it's transport layer session bound to the previous IP addresses is routinely invalidated and terminated. The Identifier-Locator split concept is one of the key enabler approaches to overcome abovementioned limitation, which has been recently employed in various mobility management solutions in mobile networks. Furthermore, employing it brings another benefits e.g., routing scalability, $\mathrm{IPv} 4 \& \mathrm{IPv} 6$ coexistence, multi-homing and efficient handover. In this section, we briefly go over some of the recent works on mobility management relying on the Identifier-Locator split concept and describe the novelty aspects of our approach compared to the previous studies.

IETF has developed several protocols e.g., Host Identity Protocol (HIP) [1], Locator/ID Separation Protoco (LISP) [40] and Multihoming Shim Protocol for IPv6 (Shim6) [81]. The HIP separates the identity of a host from its location using a pair of private-public keys. Each host is responsible for creating one or more public/private key pairs to provide identities for itself in the network. In the LISP a network architecture with a set of protocols are used to implement a new semantic for IP addressing. It creates two name spaces and uses two IP addresses: Routing Locators (RLOCs), which are topologically assigned to network attachment points and used for routing and forwarding of data packets through the network; and Endpoint Identifiers (EIDs), which are assigned to end-hosts independently from the network topology. The Shim6 uses different IP addresses as locators for data traffic forwarding, but presents the same source and destination identifier pair to transport and upper layers. For this, it uses an entity to map and translate upper-layer identifiers and locators for remote hosts. Identifier/Locator Split architecture for Multi-homing and Mobility (ILSMM) is another apprach proposed in [66] to support MN's multi-homing and mobility problem. It improves addressing model on the conventional IPv6 method by enabling one IP address to maintain both locator and identifier. Where the locator can be changed due to MN's movement, the identifier that has a lasting character and is maintained constantly (e.g., information of the interfaces). ILSMM used a new ID/Locator mapping mechanism including location managing server and locator mapping system. While, the location managing server handles the movement of mobile nodes, the locator mapping system is used to distribute the locators information. In [65], Heterogeneity Inclusion and Mobility Adapta- 
tion through ID/Locator Separation (HIMALIS) has been proposed, aimed to provide better mobility and routing scalability in the multi-homing and heterogeneous network environment. HIMALIS introduces an architecture including three networks components: home gateway (HGW), host name registry (HNR) and domain domain name registry (DNR). The HNR records IDs containing the reachability and location information of MNs. The DNR stores mapping information between domain names and the HNR data (i.e., IDs). During an MN's movement, the HGW uses HNR and DNR data to translate the locator information if the MN's locator stored in its ID is different from the one, that is appeared in the packet header. The Locator/Identifier Split Architecture (ILISA) was proposed in [61], with the aim of improving MNs handover performance. This approach introduces a new network entity, used to buffer incoming packets during a handover procedure and forward them towards the MN after completing its movement.

Compared to the previous related studies, mainly focused on the approaches for supporting MN's mobility in general mobile networks, we paid special attention to the LTE network. Our work differs from the literature, particularly in respect of the development of a new mobility management scheme relying on the same concept (i.e., Identifier-Locator split), that handles seamlessly MN's traffic steering and session continuity during its handover procedure in a decentralized LTE network architecture. We detail on the functionalities of the control and data planes, messaging, architecture and components of the proposed approach to realize its integration in the new LTE architecture. We extend the NS3-LENA simulation environment to implement an LTE network with decentralized architecture, being integrated with the proposed solution and to evaluate the functionality and performance of the developed approach.

\section{$4.2 \quad$ Approach}

In a decentralized LTE network, during a handover procedure with a S/PGW relocation, the MN's traffic forwarding and session continuity between two eNodeBs can be managed by the mechanism explained in $\S 2.4$. In this section, we present the functional approach for a mechanism to handle the MN's mobility requirements in the transport network (outside the EPC network).

The proposed approach relies on NAT technique, which is easily feasible to deploy with a low impact on the existing network implementation. This solution adopts the Identifier-Locator split concept, previously discussed in the Distributed Mobility Management (DMM) working group Internet draft [75]. The use of per-host locator's IP address, allows translation of IP address above 
the EPC network to steer the data traffic towards the MN's current location, while address translation is kept transparent to the endpoints.

In this context, Identifier refers to the IP address of MN's flow(s), allocated by the first attached S/PGW (e.g., A.X.X.1 in our scenario) and kept active after a handover. The IP address assigned from the second attached S/PGW's address pool (e.g., B.Y.Y.1) is referred to the Locator address and is used to forward downlink traffic to the MN's new position. As the S/PGWs use a pool of different IP addresses, after handover the Identifier address is topologically unrelated to the MN's new position, see Fig. 4.1.

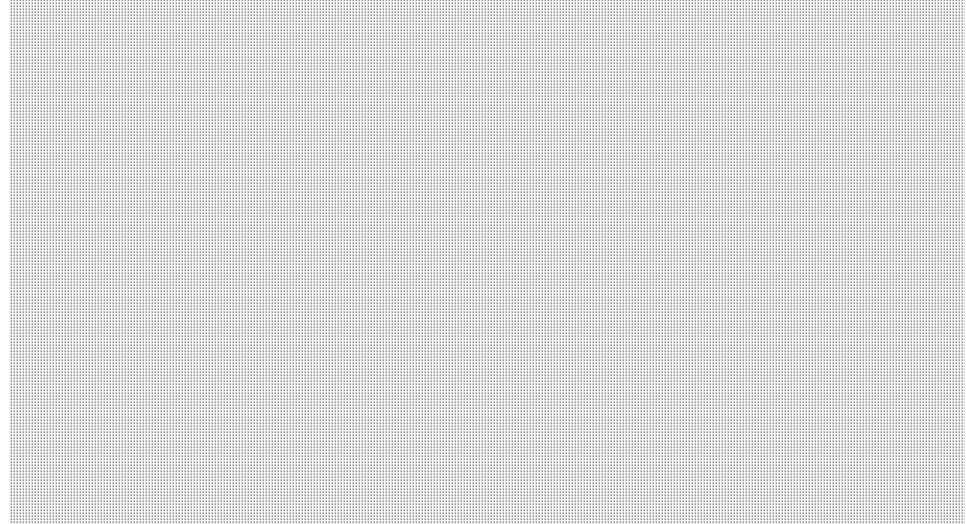

Figure 4.1: IP address translation approach to support MN's mobility on top of the EPC network in a decentralized LTE network.

\subsection{Architecture}

In a decentralized network architecture, MN's IP address is anchored at a distributed anchor point and may need to be (temporarily) maintained to keep the ongoing sessions active, when the anchor point is relocated. This may change the MN's IP address from a routable (topologically correct) into a non-routable (topologically incorrect) address at the new anchor point. In this condition, the transport network needs to steer the MN's downlink traffic to the new anchor point.

Fig. 4.2 shows architecture of the proposed approach to handle the above mentioned aspects in a decentralized LTE network. The NAT mechanism is used at the edges of the transport network to redirect MN's downlink traffic to its new location. 


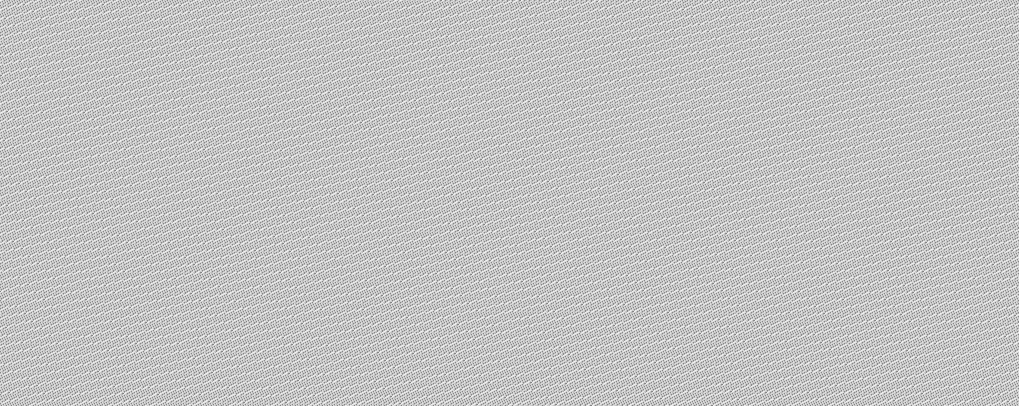

\begin{tabular}{|l|l|c|c|}
\cline { 2 - 4 } (a) Before Handover & Ingress NAT Table & S-Egress NAT Table & T-Egress NAT Table \\
\cline { 2 - 4 } & & & \\
\cline { 2 - 4 }
\end{tabular}

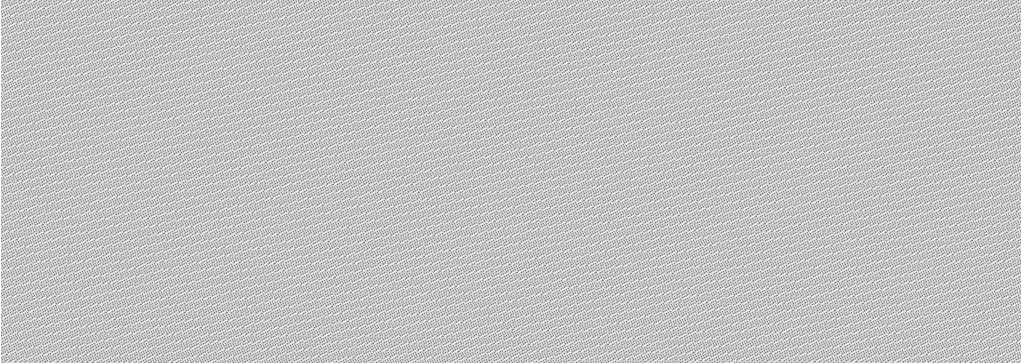

\begin{tabular}{l|l|c|c|}
\cline { 2 - 4 } (b) During Handover & Ingress NAT Table & S-Egress NAT Table & T-Egress NAT Table \\
\cline { 2 - 4 } & &
\end{tabular}

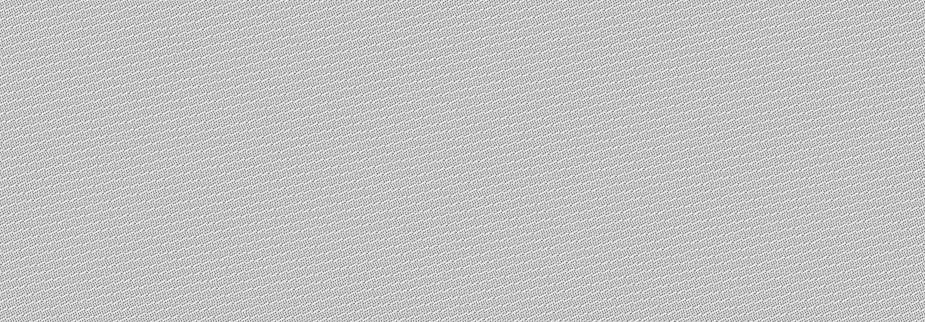

Flow IP address: T-eNodeB

A.X.X.1

\begin{tabular}{l|l|c|c|}
\cline { 2 - 4 } (c) After Handover & Ingress NAT Table & S-Egress NAT Table & T-Egress NAT Table \\
\cline { 2 - 4 } & A.X.X.1 $\rightarrow$ B.Y.Y.1 & - & B.Y.Y. $1 \rightarrow$ A.X.X.1 \\
\hline
\end{tabular}

— Plain IP $\Longrightarrow$ GTP tunnel $\quad$ - - - Additional Control Line $\quad$----- Standard Control Line

Figure 4.2: Double-NAT based traffic redirection path and architecture. 
For this, two new entities, named Ingress NAT and Egress NAT routers, perform IP address translation from Identifier $\rightarrow$ Locator address and contrariwise, respectively. As the IP address translation is applied twice in the MN's downlink path, this approach is entitled as the Double-NAT.

Assume that an MN attaches to the source eNodeB and after getting an IP address (A.X.X.1) from the pool of addresses of the source S/PGW initiates a connection to $\mathrm{CN}$ to download desired data. In the transport network a path is setup to route traffic directed to A.X.X.1 address towards the source S/PGW (the green solid-line between the Ingress NAT and source S/PGW in Fig. 4.2(a)). If $\mathrm{MN}$ dose not changed its attached $\mathrm{S} / \mathrm{PGW}$, no address translate is needed to be performed. This is due to the fact that, the flow's Identifier addresses are topologically anchored to the S/PGW, that allocates them.

When a MN is handed over to the target eNodeB, the MN's traffic forwarding and IP address continuity between two eNodBs (inside the EPC) are handled by the mechanism explained in $\S 2.4$ (the green solid-line between two eNodeBs in Fig. 4.2(b)).

After completing the handover, in the transport network (above the EPC network) the Double-NAT solution is used to redirect the flow(s), previously initiated by the $\mathrm{MN}$, to the target $\mathrm{S} / \mathrm{PGW}$. For this, the target $\mathrm{S} / \mathrm{PGW}$ allocates an IP address (e.g., B.Y.Y.1) from its address pool to the handed over MN, by receiving a specific signal from the MME, see Fig. 2.5.

This IP is not advertised to the MN and is only used as the flow's Locator address within the transport network. Next, the Ingress and Egress NAT routers take care of the correct translation of the Identifier $\rightleftharpoons$ Locator addresses to redirect the MN's flow(s) to the MN's new position. In the Ingress router, IP address of the destination in the packets is translated to the (B.Y.Y.1) and then the packets are forwarded into the transport network (the red solid-line between the Ingress and Egress NAT entities in Fig. 4.2(c))

When the packets arrive at the Egress NAT, which is placed close to the SGi interface of the target $\mathrm{S} / \mathrm{PGW}$, the changed IP address is translated back to the original destination address (A.X.X.1), and the packets are forward to the target S/PGW (the green solid-line between Egress NAT and target S/PGW in Fig. 4.2(c)).

Upon arrival of the data packets at the target S/PGW, they are processed and encapsulated into a GTP tunnel and then forwarded to the target eNodeB to be delivered to the MN via the LTE air interface.

If the MN initiates a new flow while attached to the latter S/PGW, a new address from the same pool (i.e., B.Y.Y.Y) as the Locator address, will be allocated to it.

Upon fulfilling a handover by the MN, a new NAT rule needs to be proactively defined in the Ingress and Egress NAT routers' tables. Furthermore, 
when the MN terminates the connection, its corresponding rules must be removed from the NAT tables, since this IP address may later be allocated to a newly attached MN. To accomplish these an entity entitled NAT Controller is introduced in the network. This entity is in charge of creating, updating and removing the NAT rules in the Ingress or Egress routers table. Therefore, a one-way connection is established between the NAT controller and the Ingress and Egress routers in order to add or modify the rules, upon required.

The NAT Controller needs the following information to signal the NAT routers: (i) the flow's Identifier address; (ii) the flow's Locator address; and (iii) the IP addresses of the NAT routers. During a handover procedure the target $\mathrm{S} / \mathrm{PGW}$ and its serving MME know this information. Being a control plane procedure, we chose the MME to signal the NAT Controller. Furthermore one MME can serve multiple S/PGWs, reducing the amount of connections between the EPC and NAT Controller, see Fig. 4.2.

\subsubsection{Description of Control Messages}

Two steps of signaling must be performed to create/remove a Double-NAT traffic path in the transport network, from the MME to the NAT Controller, and from the NAT Controller to the NAT routers. This section describes briefly the messages used for these procedures.

\subsubsection{Messages from MME $\rightarrow$ NAT Controller}

The MME uses two messages to signal the NAT Controller: (i) Create Path to setup a downlink path; and (ii) Remove Path to remove a previously established downlink path in the transport network. Fig. 4.3 shows the payload used for both messages. These messages carry the Identifier and Locator IP addresses. MME receives this information from the target S/PGW, via the Modify Bearer Response message, see Fig. 2.5. The I_Address denotes the Identifier address of flow(s) kept active by the MN after handover. The L_Address represents the new IP address allocated by the target S/PGW (the Locator address). If the Flag is set to 00, it indicates that the packet carries a Remove Path message, otherwise it carries a Create Path message.

\begin{tabular}{|l|l|l|}
\hline $\begin{array}{l}\text { I_Address } \\
\text { (32 bits) }\end{array}$ & $\begin{array}{l}\text { L_Address } \\
\text { (32 bits) }\end{array}$ & $\begin{array}{l}\text { Flag } \\
\text { (2 bits) }\end{array}$ \\
\hline \hline A.X.X.1 & B.Y.Y.1 & 00 \\
\hline
\end{tabular}

Figure 4.3: Payload of Create Path and Remove Path messages. 
If more than one NAT Controller be presented in the network, an specific protocol is needed to unify the NAT tables throughout the network. In this work only the case with one NAT Controller is studied.

\subsubsection{Messages from NAT Controller $\rightarrow$ NAT Routers}

The NAT Controller uses two messages to signal the NAT routers: (i) Update Rule to add/modify a rule into the NAT tables; and (ii) Remove Rule to remove a rule from the NAT tables. The payload used for both messages is shown in Fig. 4.4. These messages carry the Identifier and Locator IP addresses as well as the time that a rule is created/modified in the tables. The I_Address and $L_{-}$Address define the same parameters as mentioned for the Create Path and Remove Path messages in Fig. 4.3. If the Flag is set to 00, it indicates that the packet carries a Remove Rule message. For all the other cases it shows a Update Rule message. The Time_Stamp shows the time (in ISO8601 format [2]) that a rule in the tables is created/modified.

\begin{tabular}{|l|l|l|l|}
\hline $\begin{array}{l}\text { I_Address } \\
\text { (32 bits) }\end{array}$ & $\begin{array}{l}\text { L_Address } \\
\text { (32 bits) }\end{array}$ & $\begin{array}{l}\text { Flag } \\
\text { (2 bits) }\end{array}$ & $\begin{array}{l}\text { Time_Stamp } \\
\text { (64 bits) }\end{array}$ \\
\hline \hline A.X.X.1 & B.Y.Y.1 & 00 & 20160613T083307Z \\
\hline
\end{tabular}

Figure 4.4: Payload of Update Rule and Remove Rule messages.

\subsubsection{Control Tables}

Three types of tables are used for signaling on traffic redirection procedures in the Double-NAT approach as follows:

\subsubsection{MME's Table}

MME's table contains information about the NAT Controller(s) (e.g., the IDs, IP addresses, TCP ports) present in the network. This table is used to find the appropriate entity for signaling. The State field shows the current state (active or inactive) of the NAT Controller.

\begin{tabular}{|l|l|l|l|l|}
\hline $\begin{array}{l}\text { ID } \\
(16 \text { bits })\end{array}$ & $\begin{array}{l}\text { IP_Address } \\
(64 \text { bits })\end{array}$ & $\begin{array}{l}\text { Src_Port } \\
(16 \text { bits })\end{array}$ & $\begin{array}{l}\text { Des_Port } \\
(16 \text { bits })\end{array}$ & $\begin{array}{l}\text { State } \\
(4 \text { bits })\end{array}$ \\
\hline \hline Cont_NAT_1 & $192.168 .10 .3 / 24$ & 2342 & 2712 & Active \\
\hline
\end{tabular}

Figure 4.5: NAT Controller information table in the MME. 


\subsubsection{NAT Controller's Table}

NAT Controller has two tables containing the Ingress and Egress NAT routers information about the IDs, IP addresses, TCP ports. The State filed shows whether the NAT routers are active or inactive. The table of Egress router has an extra field titled Egr_Subnet, containing information of the sub network(s) used by the target $\mathrm{S} / \mathrm{PGW}$. This data is used by the NAT Controller to find the correct NAT routers to be signaled. To do so, a longest prefix match calculation is performed between the flow's Locator address and all the Egr_Subnet entries in the table. The matched data is always be related to the Egress router(s) connected to specific S/PGW.

NAT Controller keeps the IDs of signaled NAT routers in a specific table. The stored information is used when a rule needs to be removed from a particular NAT router(s).

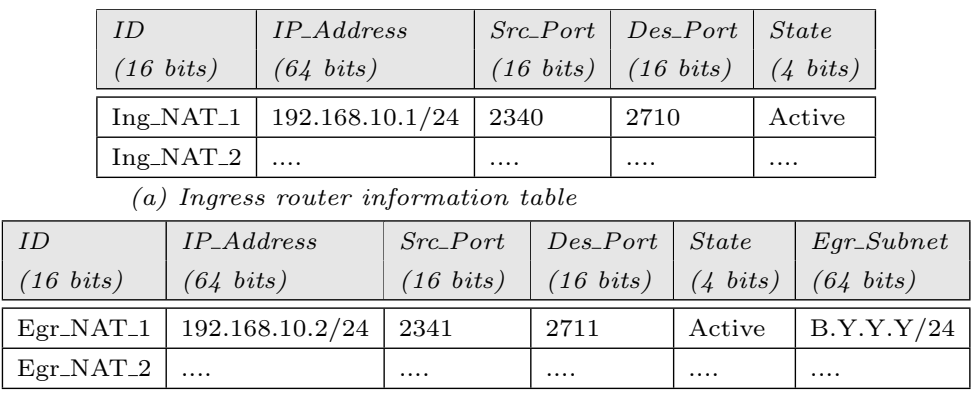

(b) Egress router information table

Figure 4.6: NAT routers information tables in the NAT Controller.

\subsubsection{NAT Routers' Tables}

The Ingress and Egress NAT routers keep per-host states in their tables to steer the MN's downlink traffic in the transport network, and towards the corresponding target S/PGW, respectively. In the Ingress router table, Local_Address and Global_Address fields contain the IP addresses used to forward MN's downlink traffic in the external network (i.e., the Internet, IMS) and in the transport network, respectively. These addresses, in order refer to the I_Address and L_Address in messages sent by NAT Controller. A combination of Local_Address and Global_Address define a rule to crate a path for MN's downlink traffic. In Egress router table, Local_Address (the $L \_A d d r e s s$ in the NAT Controller's messages) and the Global_Address (the I_Address in the NAT Controller's messages) is used to forward MN's traffic in the transport network and inside the EPC network, respectively. The Egress NAT router may be connected to more than one 
S/PGW. Therefore, an addition column (Output_Intf) is defined in the table to choose the correct output interface connected to corresponding target S/PGW. This filed refers to the interface where the Egress router is connected to the target S/PGW (with IP: B.Y.Y.101/24 in our example). The Last_Updated defines is the time when the modification is applied in the tables.

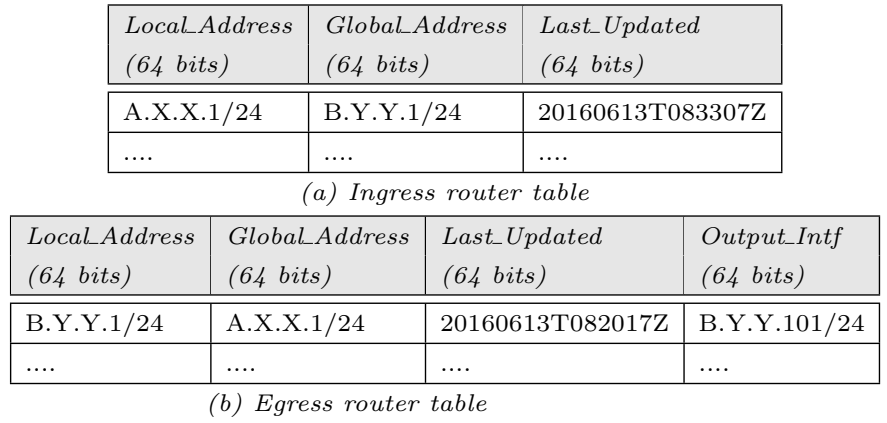

Figure 4.7: NAT routers tables.

\subsubsection{Description of Control Messages and Flow Diagrams}

This section describes the flow of control messages (see Fig. 4.8) exchanged between the EPC entities and Double-NAT components during different procedures. The description follows Fig. 4.8 from top to bottom .

\subsubsection{During Handover Procedure}

During a MN's handover procedure, in order to setup a new downlink path in the transport network, towards the MN's current location, following messages are exchanged between the EPC and Double-NAT approach entities:

(1) Target S/PGW send a Modify Bearer Response message to the MME and notifies it about the newly assigned IP address to the MN, see Fig. 2.5.

(2) MME looks in the MN's context, already stored during the attachment procedure, and retrieves the IP address of the flow(s) kept active by the MN after handover. Next, it sets the old and new IP addresses as the Identifier and Locator addresses in the Create Path message and sends it to the NAT Controller. For each MN's active IP addresses a new Create Path message is set up. 
(3) NAT Controller uses this information to construct the Update Rule message, sends it to every active NAT routers, and saves the IDs of the signaled routers.

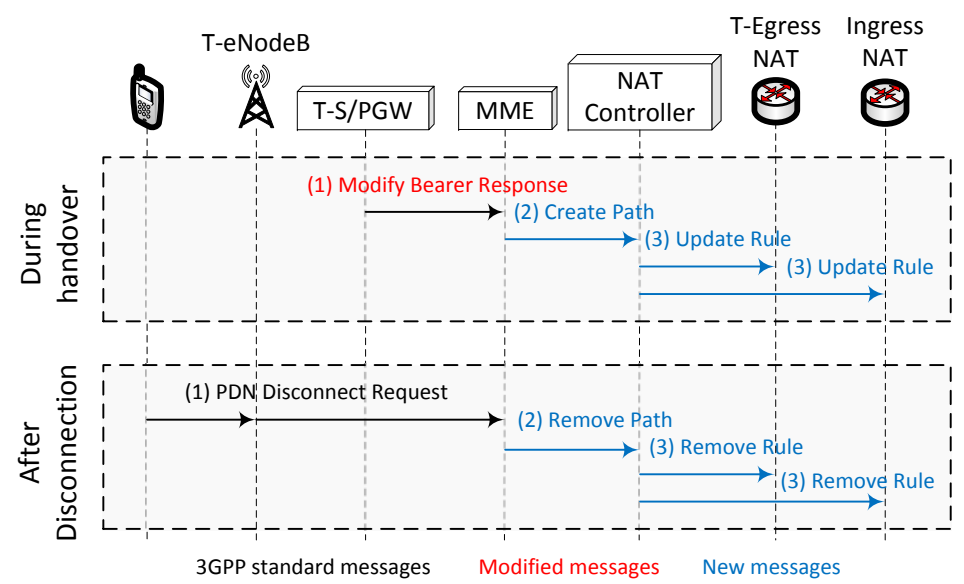

Figure 4.8: Double-NAT solution control messages flow.

Upon receiving the Update Rule message by the Ingress router, it looks up its table for an entry whose Local_Address matches to the I_Address extracted from the message. If an entry is found and the Time_Stamp of the received message is higher than the Last_Updated of the entry, the entry's filed is updated by the Time_Stamp value. Otherwise the new rule is added to the table.

Similarly, when an Egress router receives the Update Rule message, it looks up its table for an entry whose Local_Address matches with the $L \_$Address. If an entry is found and the Time_Stamp of the received message is higher than the entry's Last_Updated value, the entry's field is updated with Time_Stamp value. Otherwise the new rule is added to the table.

If Ingress router receives the Update Rule message with a substantial advance than the Egress routers, the traffic forwarding procedure can be encountered an error situation. This is due to the fact that, the downlink packets already be NATted at the Ingress router may receive at the Egress router while the related table has not been updated yet. This results in to route the packets to a wrong S/PGW. To avoid this, the message towards the Ingress router should be transmitted with a safety delay (depending on the network topology) than the one directed to Egress router. 


\subsubsection{After PDN Disconnection Procedure}

After the MN's disconnection procedure, to remove the previously established path in the transport network, following messages are exchanged between the EPC and Double-NAT approach entities:

(1) MN sends a PDN Disconnect Request message including the LBI to the MME and asks to disconnect from the network, see Fig. 2.6.

(2) MME extracts the current and previously assigned IP addresses of the MN from the MN's context and LBI. A Remove Path message is created by setting the old and new IP addresses as the Identifier and Locator addresses, and then it is sent to the NAT Controller.

(3) The NAT Controller uses this data to create a Remove Rule message, sends it to the signaled NAT routers, and removes IDs of the signaled routers from the list. When the NAT routers receive this message, they look up at the related tables for an entry whose data matches with the received information. If any entry is found, it is removed from the table.

\subsection{Setup of Simulation Study}

This section presents the evaluation scenario and implementation of the components in the simulation environment. We set up the simulation environment as realistic as possible to get reliable results, as described below.

\subsubsection{Evaluation Scenario}

The logical network topology of the evaluation scenario is the one shown in Fig. 4.2. During 20 second simulation, $120 \mathrm{MNs}$ attach to each eNodeB, initiate IP flows using the IP addresses allocated from the corespondent S/PGWs, and generate the E-UTRAN traffic according to Table 4.2. $30 \%$ of MNs from the source eNodeB, running VoIP application move to the target eNodeB at different times between 10.7 to 12.8 seconds after starting of the simulation time. The X2-based (inside the EPC) and the Double-NAT solutions (above the EPS) forward the MNs' downlink traffic to the target position.

\subsubsection{Implementation of Scenario in the NS3-LENA}

We extended the NS3-LENA simulation environment to implement the required components and to evaluate the functionality and performance of the proposed 
solution. The extensions are described below. The link of the source code for implementation of the all modules can be found in appendix B.

\subsubsection{Decentralized LTE Network}

Several modifications are needed in the existing version of NS3-LENA to implement a decentralized LTE network. In the current release only one SGW/PGW entity can be instantiated and the X2-based handover is not supported for SGW relocation. The modifications applied to overcome these limitations are discussed in the following:

\section{- Instantiation of Multiple S/PGWs}

For this, we extend the EpcHelper module of the NS3-LENA to implement two standalone EPS subsystems that use independent S/PGWs with different pool of IP addresses. The S/PGWs serve the separate eNodeBs, but use a shared MME. Each S/PGW uses its own pool of IP addresses in the same subnet as their IP address advertised outside the EPS system (i.e., the IP address of the SGi interface).

- Implementation of X2-based Handover Procedure with S/PGW Relocation

Having multiple S/PGWs in the simulation scenario, we modified the currently deployed X2-based handover procedure of the NS3-LENA to support relocation of S/PGW during the MNs handover and to realize the functions described in $\S$ 2.4. The required modification are as following:

1. In the current version of NS3-LENA, the source eNodeB forwards the MN's downlink traffic via the X2 path (in the Handover Execution phase) only if it receives a Handover Request Ack message from the target eNobeB (in the Handover Preparation phase), and the packets received before this message are dropped. To cope with this issue, we enhanced the buffer used in the eNodeBs (the EpcEndApplication) to also save the downlink packets received in the Handover Preparation phase.

2. After Handover Completion a S1-U tunnel needs to be established between the target S/PGW and target eNodeB. To do so, we implement a ModifyBearer function within the EpcHelper module to emulate the steps of (2) to (6) as described in Fig. 2.5. Next, the target S/PGW can assign a new IP address to the moving MN. This IP can be used either by the MN to establish a new IP flow or by the Double-NAT mechanism to steer the traffic within the transport network. 
3. After establishment of a new bearer in the target S/PGW, MN's downlink traffic might still be received at the source S/PGW, when the Double-NAT traffic redirection path has not been setup yet. Therefore, the source eNodeB checks every $1 \mathrm{~ms}$ (using LteEnbRrc module) if any packet(s) has been added to its buffer (the EpcEndApplication) for the moving MN. If present, the packet(s) is forwarded to the target eNodeB via the $\mathrm{X} 2$ interface.

4. Since the S11 signaling used by the MME to delete the moving MN's session(s) in the source S/PGW (Steps (9), (10) in Fig. 2.5), is not implemented yet in the NS3-LENA, a Delete Session Timer is used to terminate the X2 path established between the eNodeBs. If the Double-NAT traffic redirection path is not completed upon this time expiration, downlink data packets might be lost when received at the source $\mathrm{S} / \mathrm{PGW}$ since there is no MN's session present anymore. In our experiments the Delete Session Timer is setup to $10 \mathrm{~ms}$.

\subsubsection{Transport Network}

Transport network refers to the IP network, connecting CNs to the EPC network. The transport network topology is according to a small part of the EBONE (one of the European ISPs) network, covering The Netherlands, northeast of Belgium and north-west of Germany. This topology has been implemented in the NS3-LENA using Rocketfuel mapping engine [10]. Rocketfuel engine uses trace route techniques e.g., [90] in order to infer an ISP's routerlevel typology.

\subsubsection{NAT Function}

We used an external module developed in [6] to implement the NAT functions. This module allows to the IP flows to be established in either direction (Static $N A T$ ) or only in one direction (Dynamic NAT). It also allows to multiplex several hosts onto a single IP address via port translation. Using the Ipv 4 NatHelper entity of it, NAT capabilities can be added to a node defined in the NS3 environment (e.g., NAT routers in Fig. 4.2). Hereafter, the node can perform NAT table lookup on the incoming packets. A NAT rule can be added or remove from the NAT table of a node using a simple function call. Currently implemented model supports only the IPv4 addresses. 


\subsubsection{Control Messaging}

To implement the signaling procedures described in $\S 4.3 .3$, the UdpSockets are set up between the MME, the NAT Controller, and the NAT routers entities.

\subsubsection{MNs' Movement}

In NS3-LENA, the MNs' handover time is based on a pre-defined schedule, set up in the simulation. Therefore, no MNs' movement is needed to trigger the handover procedures. The MNs are only placed in positions at the same distances from the eNodeBs and a distribution of dwell time is used to trigger the handovers. We used the Fluid-Flow mobility model [86] to derive the average dwell time of the MNs in each S/PGW as follows;

$$
E\left[T_{d w e l l}\right]=\frac{\pi \times A}{V \times P}=\frac{\pi \times\left(\pi R^{2}\right)}{V \times(2 \pi R)}=\frac{\pi \times R}{2 \times V},
$$

where $V$ presents the velocity of a $\mathrm{MN}$, and $R, A$ and $P$ denote the radius, coverage area and perimeter of each $\mathrm{S} / \mathrm{PGW}$, respectively. As a $\mathrm{S} / \mathrm{PGW}$ relocation most likely happens for the MN that are on a highway, we used the Free Speed Distributions model [57] to compute the velocity of the MNs. We used a Normal Distribution with the (Mean $=32.1 \mathrm{~m} / \mathrm{s}$ ) and (Standard Deviation =4.33) [57]. For the sake of simplicity we assumed that the MNs move in a straight road between the S/PGWs.

\subsection{Simulation Parameters}

This section presents the setting of parameters in the simulation environment.

\subsubsection{E-UTRAN Setting}

Table 4.1 summarizes values of the configured parameters in the RAN. The values are based on the LTE release- 8 specifications, implemented in the NS3LENA.

\subsubsection{E-UTRAN Traffic}

We used the traffic mix model (see Table 4.2) specified in [60] to generate the RAN traffic. VoIP is selected as the traffic generated by the moving MNs. Other types of traffic are used to generate the RAN background traffic by the stationary MNs attached to the both eNodeBs. Only the moving MNs' traffic is forwarded to the Internet and the rest is dropped at the eNodeBs. 


\subsubsection{EPC and Transport Network Setting}

The values of parameters set up for the EPC and transport network are shown in Table 4.3. The size of buffer for the entities in the both networks is set to $\left(\frac{C \times R T T}{10}\right)[44]$. Where $C$ and $R T T$ denote the link speed and the Round Trip Time (=250 ms [44]) of the flows in the network, respectively.

Table 4.1: The E-UTRAN parameters.

\begin{tabular}{|l|l|}
\hline Parameters & Value \\
\hline \hline Uplink and Downlink bandwidth & $5 \mathrm{MHz}$ \\
Source eNodeB uplink / downlink & 2535 / 2655 MHz \\
Target eNodeB uplink / downlink & $2540 / 2650 \mathrm{MHz}$ \\
Transmission mode & $\mathrm{MIMO} 2 \mathrm{x} 2$ \\
$\mathrm{MN} /$ eNodeB transmission power & $26 / 49 \mathrm{dBm}$ \\
MN / eNodeB noise figure & $5 \mathrm{~dB}$ \\
Cell radius & $2 \mathrm{Km}$ \\
Cell distance & $4 \mathrm{~km}$ \\
\hline
\end{tabular}

Table 4.2: The E-UTRAN traffic model.

\begin{tabular}{|l|l|l|}
\hline Application & Traffic & Percentage of MN \\
\hline \hline VoIP & Real-time & $30 \%$ \\
FTP & Best effort & $10 \%$ \\
HTTP & Interactive & $20 \%$ \\
Video & Streaming & $20 \%$ \\
Gaming & Interactive real-time & $20 \%$ \\
\hline
\end{tabular}

Table 4.3: The EPC and transport network parameters.

\begin{tabular}{|l|l|}
\hline Parameters & Value \\
\hline \hline Transmission technology & Ethernet \\
MTU size & $1.5 \mathrm{~KB}$ \\
Transport / EPC network links data rate & $10 / 1$ Gbps \\
Queue scheme & Drop-tail \\
Transport / EPC network nodes buffer size & $31.250 / 3.125 \mathrm{MB}$ \\
Radius of a tracking area (TA) of each S/PGW & $50 \mathrm{KM}$ \\
\hline
\end{tabular}




\subsubsection{The EPC and Transport Networks Traffic}

We used the Poisson Pareto Burst Process (PPBP) model [28] to generate a realistic Internet traffic in the wired networks. $80 \%$ is chosen as the maximum level of link utilization for the both networks. The rest of available capacity is used to transfer the VoIP traffic and also to keep as the safety capacity. In this model, bursts arrive according to a Poisson process with mean distribution $\left(T_{o n}\right)$ and burst's flow constant bit-rate $(r)$. The length of bursts follows a Pareto distribution characterized by Hurst parameter $(H)$, typically with a value between 0.5 and 0.9 . The average number of active bursts is given by;

$$
E[n]=T_{o n} \times \lambda_{p},
$$

where $\lambda_{p}$ defines rate of the arrival bursts. Table 4.4 shows the values of the parameters used in the PPBP model to generate links utilization traffic in the wired networks.

Table 4.4: The wired networks traffic model parameters.

\begin{tabular}{|l|l|l|l|l|l|}
\hline Link speed & $\mathbf{E}[\mathbf{n}]$ & $\mathbf{r}$ & $\lambda_{p}$ & $T_{\text {on }}$ & $\mathbf{H}$ \\
\hline \hline $10 / 1$ Gbps & 40 & $200 / 20 \mathrm{Mbps}$ & 2000 & $0.002 \mathrm{~s}$ & 0.7 \\
\hline
\end{tabular}

\subsection{Performance Evaluation}

We evaluate the seamlessness of the proposed mobility support solution, using simulation experiments. That is, if no significant packet loss and delay are introduced during the MN's handover with a S/PGW relocation. For this we define the following performance metrics and present the obtained results accordingly. For this, we investigate different performance measures for the MNs performing a handover with $\mathrm{S} / \mathrm{PGW}$ relocation. In particular we study the latency of MNs' data packet delivery before and after handover ( $\S 4.6 .1$ ), CDFs of latency of the MNs' downlink data packets $(\S 4.6 .2)$, and packet loss in the redirected downlink data traffic $(\S 4.6 .3)$.

As a means of providing reliable performance results and hence insight into the system behavior, we provide mean results with $95 \%$ confidence interval by repeating the simulations for the same setting, using 10 random seeds. Note that due to partial overlap of the confidence intervals for different input values, they are presented for limited number of measurement points and graphs, for better presentation purpose. 


\subsubsection{Average Latency of Data Packets Delivery Before and After Handover}

The average latency of the data packets received by the moving MNs via the alternative downlink paths before and after handover is presented in Fig. 4.9. The graphs clearly demonstrate that the proposed solution has no considerable impact on latency of the data packets redirected to the MNs after a handover. As the placement of NAT Controller in the transport network could be critical, we also studied the impact of it's position in the network topology (see Fig. 4.2). Given that the MME uses the transport network to signal the NAT Controller, it is observed that no significant impact is appeared in the average latency after handover $(\leq 4 \mathrm{~ms})$, when distance between the NAT Controller and MME is changed from 1-7 hops.

In the simulation scenarios, the Egress NAT routers is placed in a fixed position with one hop distance to the S/PGWs. Positioning of the Ingress NAT routers has no particular constrain and mainly depends on network design. Our experiments shows that after handover, the average latency of data packets delivery has slightly variation $(\leq 6 \mathrm{~ms})$, when distance between the Egress and Ingress NAT routers is changed from 1-7 hops. This accordingly could affect the throughput of the Double-NAT solution.

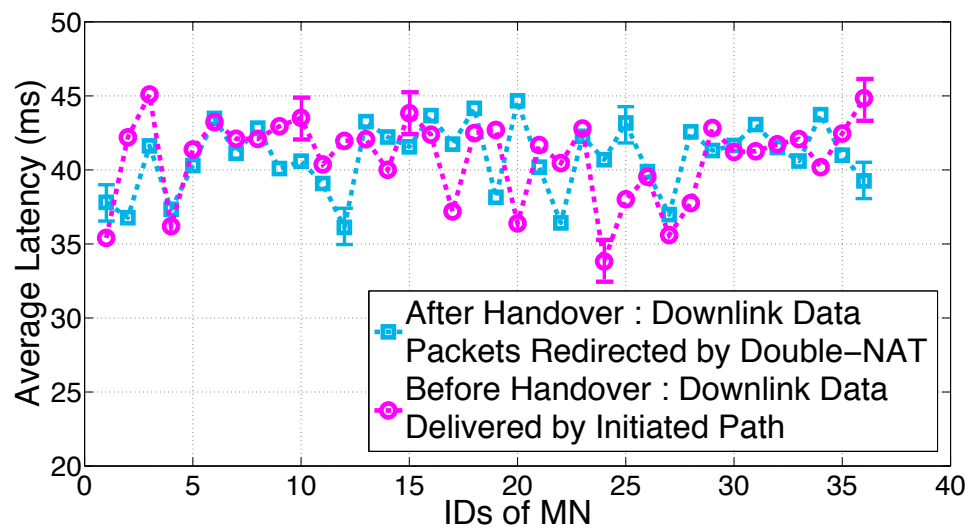

Figure 4.9: Latency of the MNs' downlink data packets delivery before and after handover procedure.

On may note that for some MNs (e.g., with ID $12 \& 36)$ the average latency after handover is notably less than before one. It is due the fact that: (i) the links utilization distribution (background and active traffic) meaningfully affects both the latencies; (ii) the number of MNs performing handover at the same (or 
the close) times impacts performance of the Double-NAT solution entities, and accordingly affects the latency after the handover.

\subsubsection{CDF of Latency of the MNs' Downlink Data Packets}

Cumulative Distribution Function (CDF) is a useful tool to derive the probability of an observed metric being above or below a certain values. Fig. 4.10 presents CDF of latency of the first data packets delivered to the moving MNs after completion of the handover. We select the first data packet (of the MNs), as it is the most delayed packet after the handover procedure. This is because its delivery time is directly influenced by the time required to establish the DoubleNAT traffic forwarding path in the transport network. This evaluation provides a better view of the seamlessness of the proposed solution. The obtained results show that the Double-NAT approach is fast enough to easily meet the maximum allowed latency of $150 \mathrm{~ms}$ [9] as a threshold for the VoIP application.

Fig. 4.10 also shows CDF of latency of the data packets forwarded, from the source eNodB to the target one, through the X2 path during the MNs' handover.

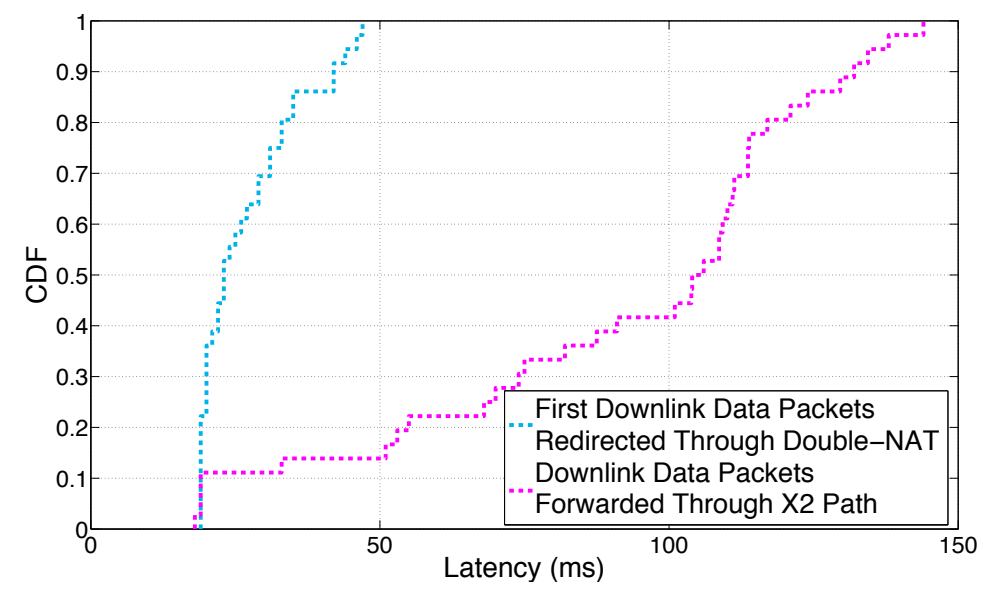

Figure 4.10: CDF of latency of the first downlink data packets redirected through the Double-NAT, and data packets forwarded via the X2 interface.

Although X2 interface is a useful feature introduced in 3GPP standards to provide a seamless mobility to the MNs moving between the neighboring eNodBs, the graph shows that the Double-NAT approach significantly outperforms the X2 interface. Therefore, if a function to predict the next location of MN (e.g., target eNodeB), would be present in the network e.g., [73], Double-NAT 
traffic redirection path can be set up a priori to avoid the usage of $\mathrm{X} 2$ data forwarding.

\subsubsection{Packet Loss Ratio in the Redirected Downlink Data Traffic}

The X2 path is used to forward the MNs' downlink data during the handover procedure, and also after when the Double-NAT traffic redirection path is not set up yet. In the latter case, provided that the MN's session has not been deleted yet by the MME (see Fig. 2.5), the downlink packets are still being received at the source $\mathrm{eNodB}$ are delivered to the target one via the $\mathrm{X} 2$ path. By allowing the use of X2 path for $10 \mathrm{~ms}$ after the handover procedure (by setting Delete Session Timer $=10 \mathrm{~ms}$ in the MME [20]), no loss is presented in the MNs' data. It implies that $10 \mathrm{~ms}$ is enough to cope with the latency, needed to establish the Double-NAT traffic forwarding path.

Mobile network operators may wish not to use the X2 path after the MNs' handover (setting Delete Session Timer $=0$ ).

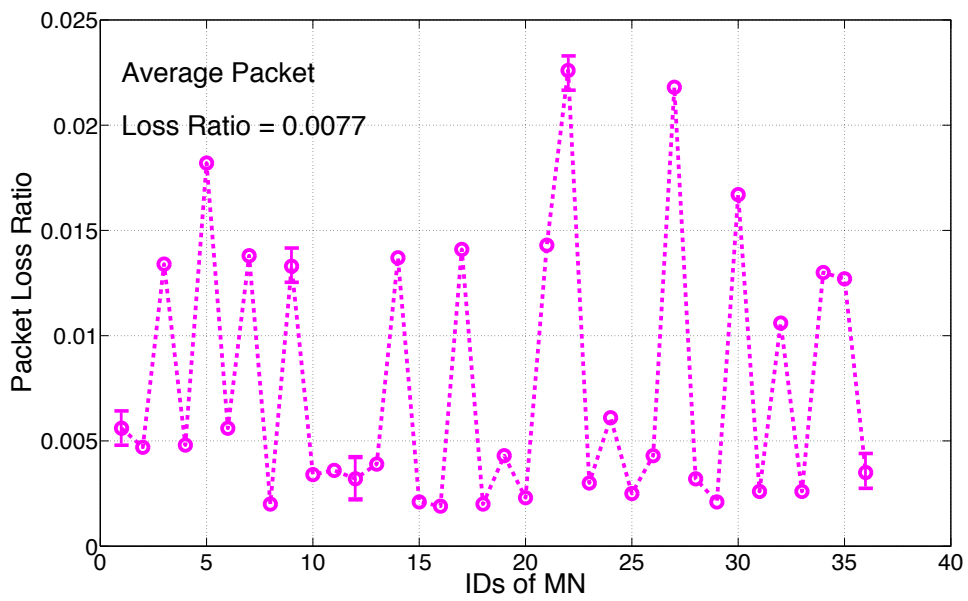

Figure 4.11: The MNs' downlink data packets loss ratio without using the X2-path traffic redirection.

In this case the moving MN's session is removed from the source S/PGW, upon the $\mathrm{S} 1$ bearer is initiated at the target $\mathrm{S} / \mathrm{PGW}$. This situation may results in some packet loss for the moving MNs. Fig. 4.11 shows the downlink packet loss ratio for the moving MNs, without using the X2-based data forwarding capability in the network. 


\subsubsection{Control Messaging Overhead of the Proposed Solu- tion}

In the proposed approach, to set up a traffic redirection path, several messages must be exchanged between the MME and the Double-NAT entities, see Fig. 4.8). Assuming that only the messages of the NAT Controller towards the NAT routers are sent through the transport network, Fig. 4.12 shows load of the Double-NAT messages on the network, as a function of the number of the MNs handed over to a new S/PGW. It also shows the load of messages of the GTP [21] and PMIP [20] protocols, during the MNs handover, given a SGW relocation, in the existing LTE network. It is clear that the Double-NAT outperforms the other mechanisms and has lower impact on the network load.

For each approach, the graph shows the load of control messaging, in terms of the number of bytes traversed through the core network during the MNs' handover procedure. One may note that, as the number and size of the messages used for each $\mathrm{MN}$ is constant, the graphs grow linearly by increasing the number of MNs performing a hand over among the SGWs (S/PGWs).

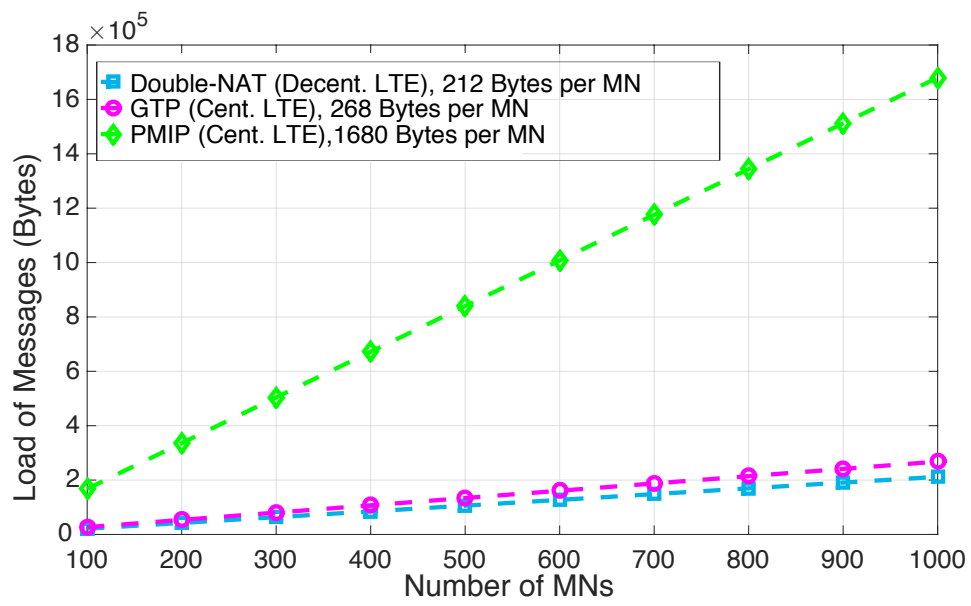

Figure 4.12: Load of the MN's mobility support messages using different approaches in the centralized and decentralized LTE architectures.

\subsection{Concluding Remarks}

In this chapter, we have presented a novel mechanism to support IP traffic continuity for the flow(s) kept active by the MNs performing handover with $\mathrm{S} / \mathrm{PGW}$ relocation in a decentralized LTE architecture. Our solution is based 
on the NAT technique, which is widely used in the current Internet. Therefore, integration of the NAT functions into several network routers is easily feasible with a minimal overhead and complexity. Handling NAT tables on the NAT routers for steering the MNs' traffic outside the EPC network can be performed using small size messages, with negligible impact on the network load. Besides this, deploying the NAT routers only at the edges of the transport network makes our solution to be deployable at a large scale. Detailed simulation shows that the proposed solution is fast enough in setting up the traffic redirection path and readily meets the latency requirement, considering the maximum allowed delay threshold for real-time applications (e.g., VoIP). The main challenge in the Double-NAT solution is the placement of its entities in the transport network. As described already, the Egress NAT routers need to be placed at one hop distance to the S/PGWs entity. Although positioning of the Ingress NAT routers as close as possible to the Internet or IMS, seems the most logical solution, some network topologies may take advantages of having a close distance between the Ingress and Egress NAT routers. The placement of the NAT controller can be influenced by both EPC deployment and Ingress NAT routers positioning. A trade-off for placing the NAT controller close to the MME or the Ingress NAT routers may need to be determined. 



\section{Network Layer Approach, SDN-based Solution}

In this chapter we present two network layer based mobility support approaches relying on the SDN paradigm, which is also one of the most important candidate technologies to realize future mobile networks (e.g., 5G). The proposed solutions can handle traffic redirection and seamless IP address continuity for the MNs moving among the distributed S/PGWs (see § 2.3) in a decentralized LTE architecture. This chapter details the architectures, functions and components of the proposed solutions, and presents the performance metrics and the obtained results of the evaluation. The publications related to this chapter are [67, 71, 68].

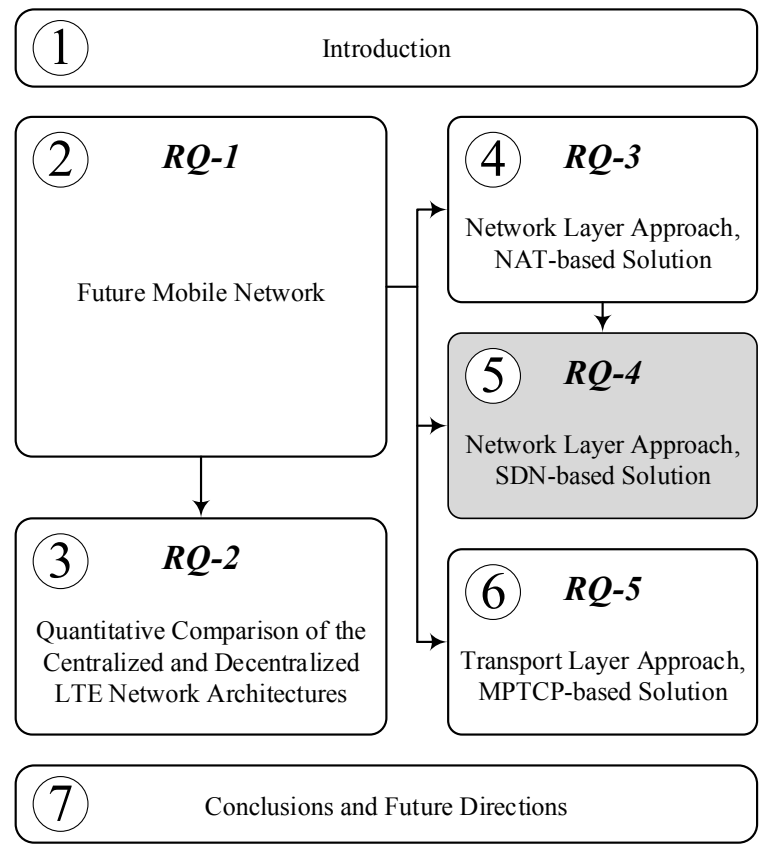


This chapter is organized as follows:

- Section 5.1 reviews briefly recent related work and states the contribution of this chapter.

- Section 5.2 describes the functional approach of the proposed solutions.

- Section 5.3 presents the architectures, components, and control messages of the proposed solutions.

- Section 5.4 describes the evaluation scenario and implementation of its components in the NS3-LENA simulation environment.

- Section 5.5 defines setting of the parameters in the simulation environment.

- Section 5.6 defines the performance metrics for the validation and presents and discusses the obtained results accordingly.

- Section 5.7 concludes this chapter. 


\section{$5.1 \quad$ Related Work}

In this section we briefly look at the recent related works and state how our work differs from the literature.

Profiting from the key potential of SDN on detaching the control and data planes, several studies have been performed mostly aimed to improve e.g., flexibility, elasticity, efficiency, scalability and adaptability of the mobile network architectures. In this section we look at some recent works on SDNbased mobility management and in particular state how our approaches differ from the previous studies.

In [29] a centralized SDN-based mobility management approach with X2 interface forwarding scheme was proposed to handle MNs mobility in an enterprise femtocell network. The study aimed to show how employing of the SDN in mobile networks can cause on reducing total amount of handover signaling cost by minimizing the number of exchanged signals as well as by allowing a local forwarding path, computed in a centralized manner. The work in [98] proposed an SDN based traffic forwarding approach for small cell backhaul LTE networks. It used a centralized controller for the control plane within the radio access network and the data plane in the core network. A analytical model has been applied in this study for performance evaluation and to show the benefits of SDN on improvement the signalling cost and data delivery delay. The authors in [100] have presented some challenges in IP mobility management schemes, such as triangular routing and handover inefficiency, and proposed a centralized approach with extension of OpenFlow interface in order to alleviate triangle routing issue as well as protocol complexity for inter-domain mobility management. In [80], the authors proposed a SDN-based DMM solution to address sub-optimal routing and inter-domain tunneling issues. They used a numerical and prototype implementation to compare scalability and performance of the developed approach with legacy DMM. The work in [76], investigated the SDN paradigm on redesigning the LTE backhaul and looked into its potential gains in the mobility management key metrics e.g., energy consumption at MN, signaling overhead and handover delay. Authors in [25], presented a new concept for mobile network architecture, leveraging NFV, SDN, and cloud technologies to solve the challenges of resource, traffic and mobility management faced by the legacy mobile networks. In [87], a SDN-based architecture was proposed to enable the use of DMM concepts to deploy fast, flexible, reliable, and scalable mobility management mechanisms in general heterogeneous wireless networks. An SDN-based IP mobility management scheme considering inter-domain handovers, has been proposed in [56]. This solution introduced two entities (functions) in order: (i) to find the MN's target domain for choosing the appropriate SDN controller(s) to notify them about MN's communication 
information; (ii) to calculate an optimum route for MN's traffic to be forwarded between source and destination domains. In [33], an SDN-based scheme was proposed to take optimal decisions related to establishing tunneling points in the network. In this approach, SDN controller compiles the optimal configuration decisions into traffic forwarding rules and then sends them to the switches for tunnel implementation. This work, gained the advantage of the global view of the network provided by SDN controller to optimally decide the placement of tunneling points in the network, as a function of optimal route to the MNs and as a function of load on prospective tunneling point routers for load balancing.

Most of the previous related studies, mainly focused on the SDN-based mechanisms, targeting to improve handover procedure's signaling cost in the general mobile networks, to enhance optimal routing traffic path in the MIP and legacy DMM approaches, or to manage MNs mobility over the heterogeneous wireless networks. Our work differs from the literature, as we paid special attention to the LTE network and develop two new SDN-based mobility management schemes, enabling seamless traffic forwarding and session continuity for the MNs, handed over between the distributed anchor points in a decentralized LTE network architecture. We detail on the functionalities of the control and data planes, messaging, architecture and components of the proposed approaches to realize their integration in the new LTE architecture. We extend the NS3-LENA simulation environment to implement a LTE network with decentralized architecture, being integrated with the proposed solutions and to evaluate the functionality and performance of the developed approaches.

\subsection{Approach}

In a decentralized LTE network, during a handover procedure with a S/PGW relocation, the MN's traffic forwarding and IP address continuity between two eNodeBs can be managed by the mechanism explained in $\S 2.4$. In this section, we present the functional approach for two other mechanisms to handle the MN's mobility in the transport network (outside the EPC network).

The proposed schemes rely on SDN, offering a logical centralized control model and making the network more manageable and adaptable. OpenFlow is the most common protocol used in SDN [7] and enables accessing the forwarding plane of the OpenFlow-enabled switches. Using it, the switches can be (re)configured according to the requirements of the network services. The OpenFlow-based switches may work in two modes: the OpenFlow-only or OpenFlow-hybrid.

In the first mode the SDN Controller makes the forwarding decision for every packet in the network and the incoming data traffic always goes through the 
switch's flow-tables pipeline. However, the OpenFlow-hybrid switches support both the OpenFlow operations and the normal routing (L3) and switching (L2) functions. Therefore, in the latter case the SDN Controller may only make the forwarding decision for certain packets or flows depending on the application requirements, and steering of the remaining traffic can be handled by the conventional routing (switching) operations. Based on the features and capabilities offered by the OpenFlow switches, we develop two solutions to support the MNs' mobility above the EPC in a decentralized LTE network. The proposed solutions differ in the sense that they use the OpenFlow-only or OpenFlow-hybrid switches for the implementation. The first approach ( $\S 5.3 .1$ ) is based on updating the flow's table(s) of the switches, without any IP address translation or modification in the flow's packets. The second solution $(\S 5.3 .2)$ relies on the modification of the headers of the flow-specific packets on the switches. These procedures are easily feasible using the set of features offered by the OpenFlow protocol.

\subsection{Architecture}

In a decentralized network architecture, the MN's IP address is anchored at a distributed anchor point and may need to be (temporarily) maintained to keep the ongoing sessions active, when the anchor point is relocated. This may change the MN's IP address from a routable (topologically correct) into a non-routable (topologically incorrect) address at the new anchor point. In this condition, the transport network needs to steer the MN's downlink traffic to the new anchor point. Fig. 5.1 and Fig. 5.2 show the architectures of proposed approaches to handle the above mentioned aspects in a decentralized LTE network. We discuss this in more detail below.

\subsubsection{Transport Network with OpenFlow-Only Switches}

Fig. 5.1 shows the implementation architecture for the first solution, where the transport network is set up using the OpenFlow-only switches. In this scenario the SDN Controller has one-way connections to all the switches and manages them to redirect the MNs' traffic. Due to the lack of L3 routing functionality in the transport network switches, the MNs' downlink paths towards the S/PGWs need to be set up by the SDN Controller through updating the flow tables in the switches.

Assume that an MN attaches to the source eNodeB and gets an IP address allocated from the source S/PGW (e.g., A.X.X.1) to inquire some data from the CN. The MME notifies the SDN Controller about this. 


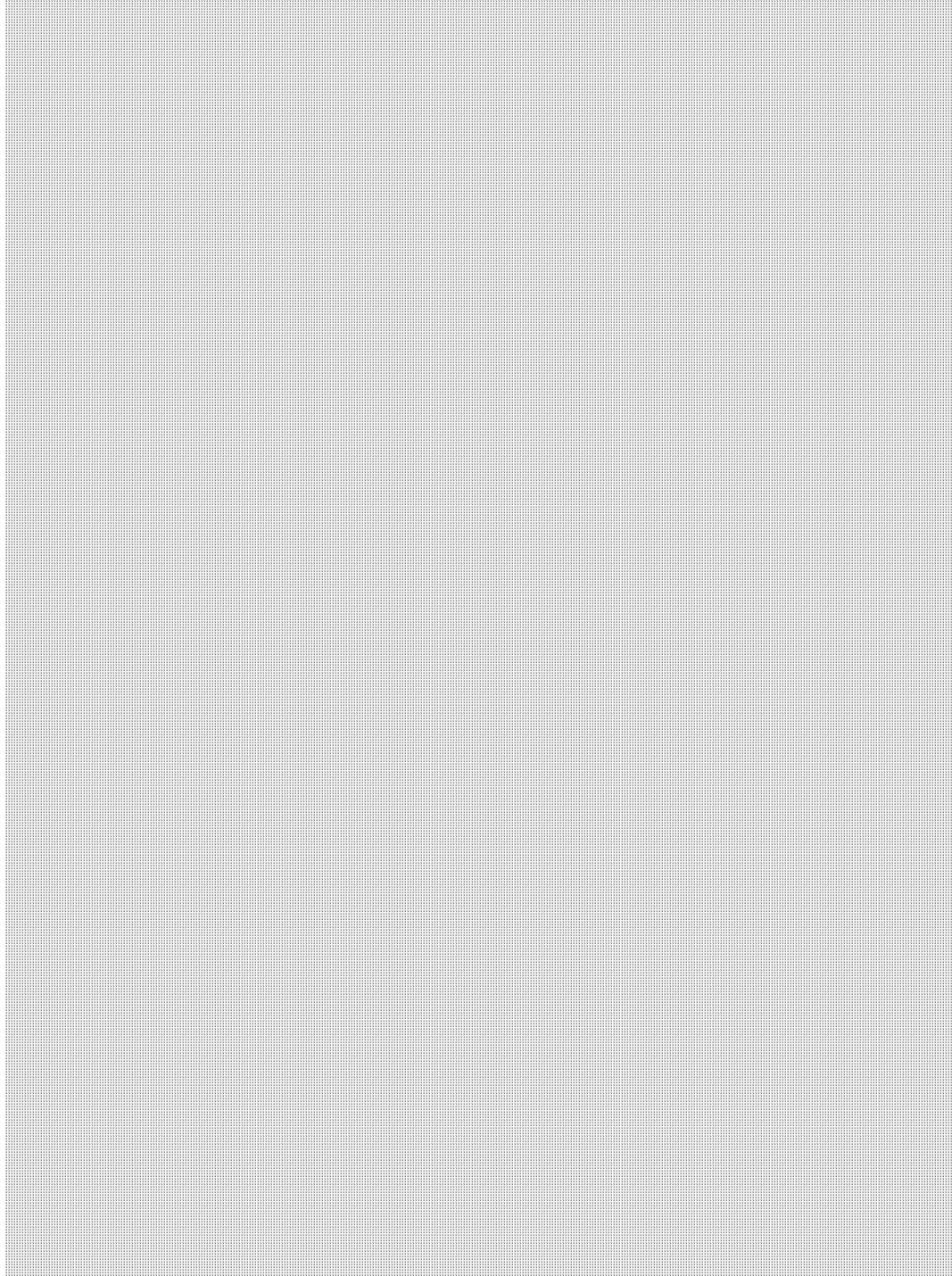

— Plain IP — GTP tunnel - - - Additional Control Line ------Standard Control Line

Figure 5.1: SDN-based traffic redirection using OpenFlow-Only switches. 
Accordingly, the SDN Controller sends a Modify-State message [7] to all the switches to add an Output action to set up a specific route for the MN's flow(s) towards it's current S/PGW. This action is added to the set of actions for the flow(s) belonging to address (A.X.X.1), and specifies the port on the switch(es) that the packets must be routed through e.g., the ports with number (1) (see Fig. 5.1). Upon receiving the MN's downlink packets in the transport network (i.e., Ingress OF), the switch looks up the IP header of the packets to find a match in its flow tables (using the <ingress port, source IP address, destination IP address $>$ information). Following the Output action added by the SDN Controller, the appropriate interface (i.e., port (1)) is selected as the output port to send the received packets. The same procedure is repeated at the other switches of the transport network to forward the MN's downlink traffic to the source S/PGW (the green solid-line between the Ingress OF and source $\mathrm{S} / \mathrm{PGW}$ in Fig. 5.1(a)).

During a handover procedure, the MN's downlink traffic forwarding between the source and target eNodeBs is handled by the procedure presented in $\S 2.4$ (the green solid-line between two eNodeBs in Fig. 5.1(b)).

When the $\mathrm{MN}$ is handed over to the target eNodeB, in transport network the Output actions of the OpenFlow switches are proactively modified to forward the MN's traffic to the correct target S/PGW. To accomplish this the SDN Controller sends a Modify-State message to the appropriate switches in order to add/modify the action set for the desired flow(s) accordingly (i.e., choosing interface (2) as the output port). Next, a similar procedure as described for setting up the MN's downlink initial path (before handover), is fulfilled in the switches to forward the MN's traffic to the new position (the green dashed-line between the Ingress OF and target S/PGW in in Fig. 5.1(c)).

Furthermore, a Modify-State message is also sent by the SDN Controller to the switches located on the MN's downlink initial path, to remove the previously added flow(s) from their flow tables. When the MN terminates the flow(s), the corresponding entries must be removed from the switches' flow tables.

\subsubsection{Transport Network with OpenFlow-Hybrid Switches}

In this solution, steering of the MN's downlink traffic is realized by the SDN Controller, managing only the Ingress and Egress switches of the transport network. Traffic forwarding between the Ingress and Egress points in the core part, is fulfilled via L3 routing in the switches (which can also be replaced with normal IP routers), instead of flow forwarding (see Fig. 5.2). 

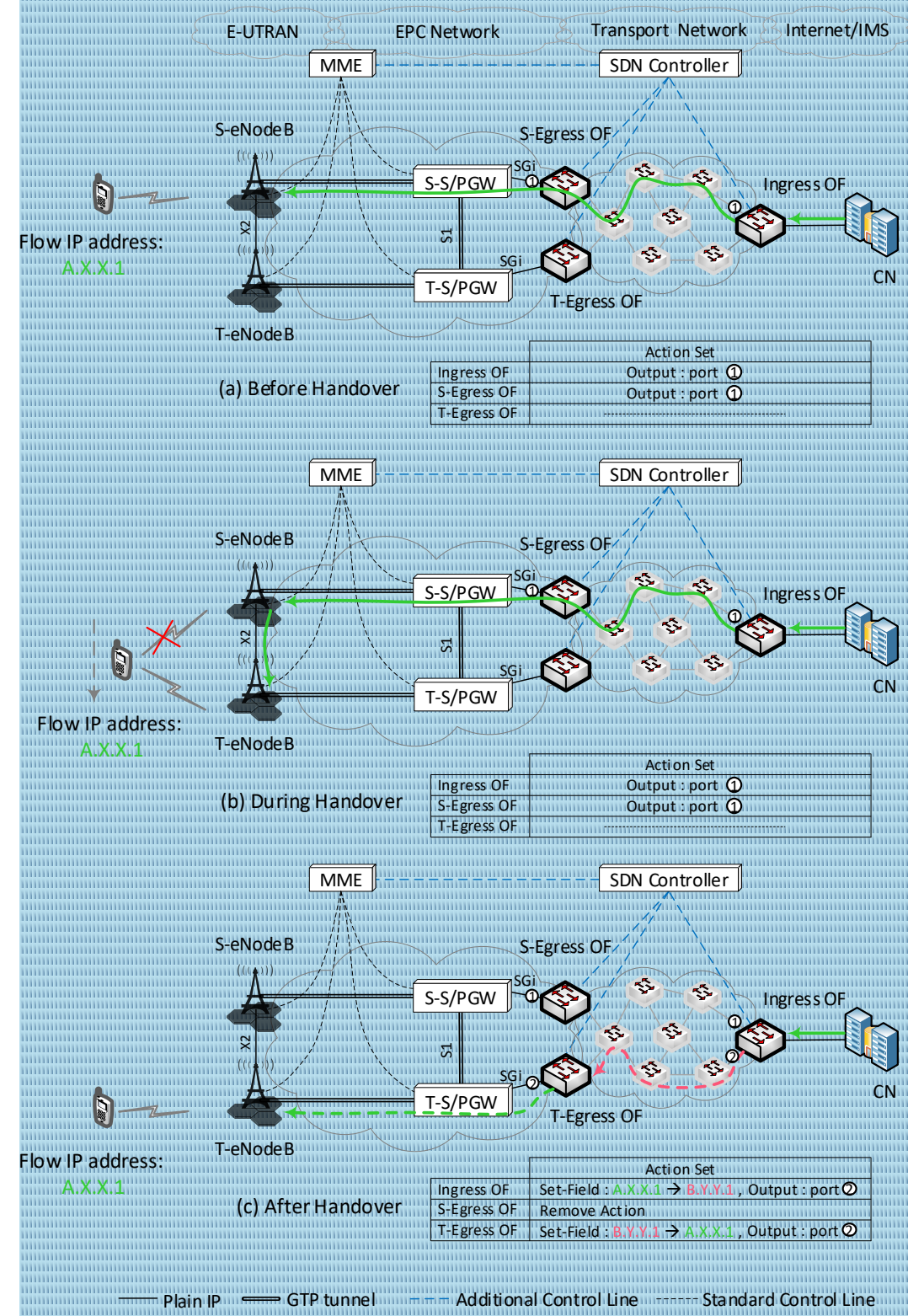

Figure 5.2: SDN-based traffic redirection using OpenFlow-Hybrid switches. 
In this scenario, the SDN Controller sends a Modify-State message to the Ingress and source Egress switches upon being informed by the MME about the newly attached MN to the source S/PGW. Accordingly, an Output action is added to the flow tables of the Ingress and source Egress switches. This action specifies the appropriate port (e.g., the ports with number (1), Fig. 5.2) to forward incoming downlink traffic to the relevant switch(es) in the transport network (from the Ingress switch) and the correct S/PGW (from the source Egress switch). Within the transport network, the MN's downlink traffic forwarding is fulfilled via an L3 routing scheme (the green solid-line between the Ingress $\mathrm{OF}$ and source $\mathrm{S} / \mathrm{PGW}$ in Fig. 5.2(a)).

During the MN's handover procedure, traffic forwarding between two eNodeBs is handled by the same mechanism as explained in the previous section.

After completing the handover, the target $\mathrm{S} / \mathrm{PGW}$ allocates an IP address (e.g., B.Y.Y.1) from its address pool to the MN (see $\S 2.4$ ). This address is not advertised to the MN and is only used to steer the MN's downlink traffic within the transport network. For this, first the SDN Controller is notified by the MME about the MN's primary IP address (i.e., A.X.X.1) allocated by the source S/PGW and its new IP address (i.e., B.Y.Y.1) allocated by the target S/PGW . Next, the SDN Controller uses this information to construct the Modify-State messages and sends them to the Ingress and target Egress switches in order to add/modify the Output and Set-Field actions [7] in the flow's set of actions. The Set-Field action in the Ingress switch, specifies the change of the MN's old IP address (A.X.X.1) $\rightarrow$ new one (B.Y.Y.1) in the incoming packets. The packets are then forwarded to the transport network through the proper port (e.g., port number (2) specified by the SDN Controller (using the Output action). Afterwards, within the transport network, data forwarding towards the target Egress switch is fulfilled via a normal IP routing procedure (the red dashed-line between the Ingress OF and target Egress OF in Fig. 5.2(c)).

Upon receiving the packets, the target Egress switch uses the Set-Field instruction to reverse the modified packets' header to the original one (translating back the IP: A.X.X.1 $\rightarrow$ B.Y.Y.1). Next, the incoming data packets are forwarded to the correct target S/PGW via the port determined by the SDN Controller (e.g., port number (2), Fig. 5.2), as the Egress switch may connect to more than one $\mathrm{S} / \mathrm{PGW}$ (the green dashed-line between the target Egress OF and target $\mathrm{S} / \mathrm{PGW}$ in Fig. 5.2(c)).

In both approaches $(\S 5.3 .1$ and $\S 5.3 .2)$, upon arrival of the data packets at the target $\mathrm{S} / \mathrm{PGW}$, they are processed and encapsulated into a GTP tunnel and then forwarded to the target eNodeB to be delivered to the MN via the LTE air interface.

During a handover procedure the MME knows the IP address of the active flow(s) after handover and the new IP address allocated by the target S/PGW. 
Therefore, in both approaches, we chose the MME to signal the SDN Controller about the required information.

An MN may have multiple IP addresses (or multiple active flows per address). In such a case, in order to decrease number of the flows' redirection paths, they can be initiated based on the IP (or MAC) address of SGi interface of the MN's current S/PGW. For this, the flow matching in the Egress switches can be performed, in order to distinguish the packets belonging to different flows, based on a combination of the source (CN) IP address and the transport layer ports. In all the other switches flow matching can be done only based on the destination (SGi) IP address of the incoming packets. Detailed information about the flow matching can be found at $\S 5.3$ of [7].

\subsubsection{Description of Control Messages}

According to our proposed schemes, two steps of signaling must be performed to create/remove traffic redirection paths in the transport network (see Fig. 5.3). This section describes briefly the messages used for these procedures.

\subsubsection{Messages from MME $\rightarrow$ SDN Controller}

The MME uses two messages to signal the SDN Controller: (i) Create Path message is used to set up a new downlink path; and (ii) Remove Path message is used to remove the previously established downlink path in the transport network. These messages may carry the IP address of the MN's assigned by the source S/PGW (during the PDN connection procedure), the IP address of the MN's active flow(s) after handover and/or the new IP address allocated by the target S/PGW (during the handover and after the PDN disconnection procedure). In different procedures, the MME may receive this information via the Create Session Response, Modify Bearer Response, or PDN Disconnection request messages (see Fig. 5.3).

\subsubsection{Messages from SDN Controller $\rightarrow$ OpenFlow Switches}

The SDN Controller uses a set of Modify-State messages to add/modify SetField and/or Output action(s) in the flow's set of actions in the corresponding switches. The action(s) and the switches to be messaged depend on the implementation of the transport network. A Modify-State message may carry the MN's old and/or new IP addresses and/or the ID of the output interface in the switches (see Fig. 5.1 and Fig. 5.2). 


\subsubsection{Control Messages Flow Diagram}

This section describes the flow of control messages exchanged between the EPC and SDN components during different procedures. The description follows Fig. 5.3 from top to bottom .

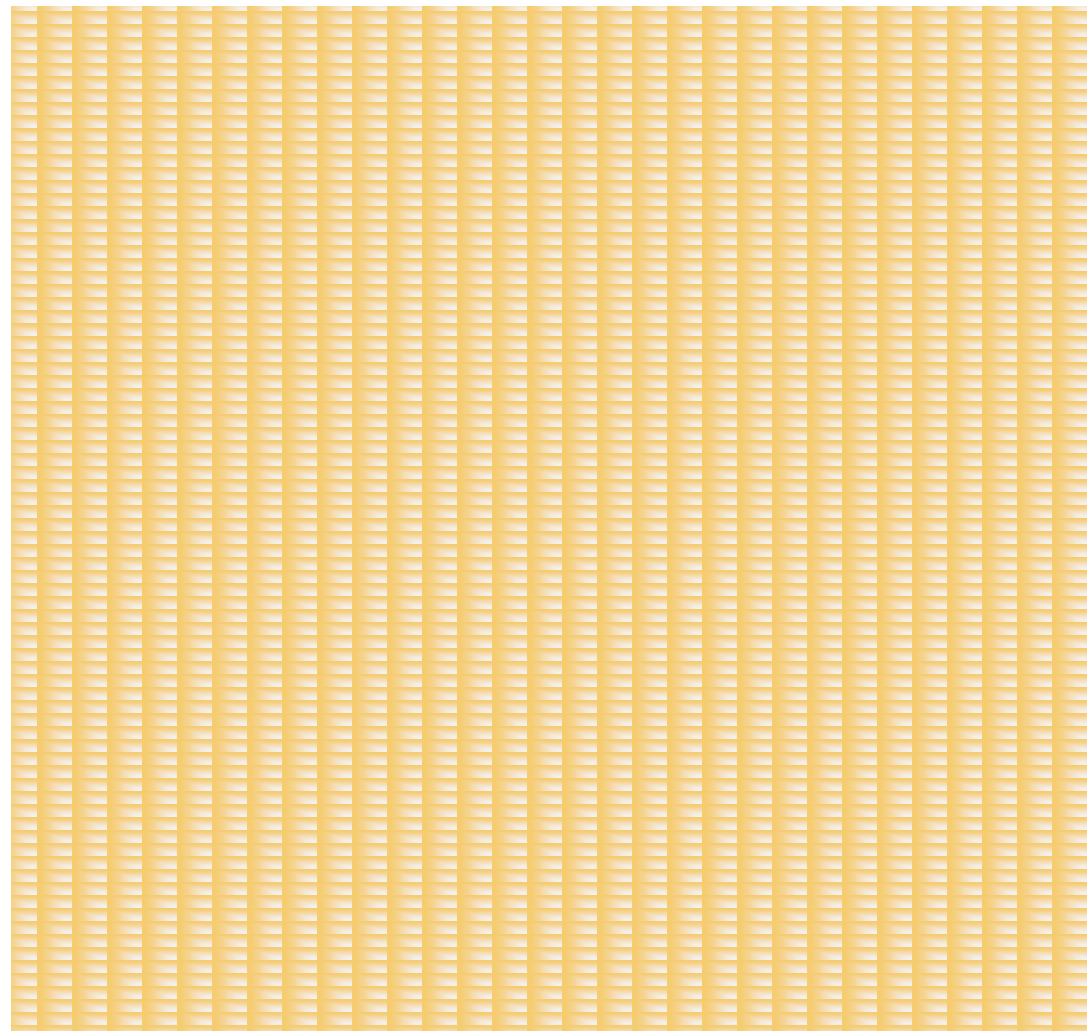

Figure 5.3: Control messages flow diagram of the SDN-based solutions.

\subsubsection{During PDN Connection Procedure}

After receiving the MN's PDN Connection Request message by MME, in order to establish the initial path for the MN's downlink traffic, following messages are exchanged between the EPC and SDN-based approaches entities:

(1) the MME sends a Create Session Request to the source S/PGW and asks to establish a new bearer to the attached MN. In the reply, the source 
S/PGW sends the Create Session Response message and notifies the MME about the assigned IP address to the MN.

(2) the MME looks at the MN's context and retrieves the allocated IP address to construct a Create Path message. Accordingly, the message is sent to the SDN Controller.

(3) the SDN Controller uses this information to create the Modify-State messages and sends them to the relevant switches (based on the different approaches) in the transport network. Upon receiving the Modify-State messages, the switches add new action(s) to the corresponding flow's set of actions.

\subsubsection{During Handover Procedure}

During a handover procedure, in order to setup a new downlink path in the transport network towards the MN's current location, the following messages are exchanged between the EPC and SDN-based approaches entities:

(1) the target S/PGW sends the Modify Bearer Response message to notify the MME about the newly assigned IP address to the MN (see $\S 2.4$ ).

(2) the MME looks also at the MN's context and retrieves the IP address of the MN's active flow(s) after handover. Next, using the new and/or old IP addresses, it makes a Create Path (or Remove Path) message and sends it to the SDN Controller.

(3) the SDN Controller uses this information to construct the Modify-State messages and sends them accordingly to the relevant switches based on the different approaches applied in the transport network. Upon receiving the ModifyState messages, the switches add new action(s) to the corresponding flow's set of actions in order to set up the MN's downlink traffic redirection path (or to remove the previously established downlink path) in the transport network.

\subsubsection{After PDN Disconnection Procedure}

After the MN's disconnection procedure, in order to remove the previously established path in the transport network, the following messages are exchanged between the EPC and SDN-based approaches entities:

(1) the MN sends a PDN Disconnect Request message including the LBI (Linked EPS Bearer ID) to the MME and asks for disconnecting from the 
network (see $\S 2.4)$.

(2) the MME extracts the current and previously assigned IP addresses of the MN from the MN's context and LBI. A Remove Path message is created using this information, and then it is sent to the SDN Controller.

(3) The SDN Controller uses this data to create the Modify-State messages and then forwards them to the already signaled OpenFlow switches. When the switches receive this message, they look up at the related flow's tables for an entry whose data matches with the received information. If any entry is found, the action(s) (specified in the received message) are removed from the set of actions of the terminated flow.

\subsection{Setup of Simulation Study}

This section presents the evaluation scenario and implementation of the components in the simulation environment. We set up the simulation environment as realistic as possible to get reliable results, as described below.

\subsubsection{Evaluation Scenario}

The logical network topology of the evaluation scenarios are the ones shown in Fig. 5.1 and Fig. 5.2. During 20 second simulation, $120 \mathrm{MNs}$ attach to both eNodeBs and generate the E-UTRAN traffic according to Table 4.2 . 30\% of MNs from the source eNodeB, running VoIP application move to the target eNodeB at different times between 10.51 to 12.96 seconds after starting of the simulation time. The X2-based (within the EPC) and the SDN-based solutions (outside the EPS) forward the MNs' downlink traffic to the target position.

\subsubsection{Implementation of Scenario in the NS3-LENA}

We extended the NS3-LENA simulation environment to implement the required components and to evaluate the functionality and performance of the proposed solutions. The extensions are described below. The link of source code for implementation of the all modules can be found in appendix B.

\subsubsection{Decentralized LTE Network}

This implementation is the same as the one described in $\S$ 4.4.2.1. 


\subsubsection{Transport Network}

The implementation of the transport network is the same as the one described in $\S 4.4 .2 .2$.

\subsubsection{SDN Function}

We used the OpenFlow Software Implementation Distribution (OFSID) library, already presented in the NS3 stable releases to implement the SDN functions. Using the OpenFlowSwitchNetDevice and OpenFlowSwitchHelper entities of it, OpenFlow switch capabilities can be added to a node defined in the NS3 environment. The SDN Controller functions can be added under the of namespace using of:: Controller. More Information can be found in [8].

\subsubsection{Control Messaging}

To implement the control messaging procedures described in $\S 5.3 .3$, the $U d p$ Sockets are set up between the MME, the SDN Controller, and the OpenFlow switches entities.

\subsubsection{MNs' Movement}

The implementation of MNs' movement is the same as the one described in $\S 4.4 .2 .5$.

\subsection{Simulation Parameters}

This section presents the setting of parameters in the simulation environment.

\subsubsection{E-UTRAN Setting}

The configuration of the parameters for E-UTRAN is the same as the one defined in $\S 4.5 .1$.

\subsubsection{E-UTRAN Traffic}

The configuration of the parameters for this section is the same as the one defined in $\S$ 4.5.2.

\subsubsection{EPC and Transport Network Setting}

The configuration of the parameters for EPC and transport network is the same as the one defined in $\S$ 4.5.3. 


\subsubsection{The EPC and Transport Networks Traffic}

The configuration of the parameters for this section is the same as the one defined in $\S$ 4.5.4.

\subsection{Performance Evaluation}

In this section, using the simulations specified above, we evaluate the seamlessness of the proposed mobility support approaches. They are, if no significant delay and packet loss are introduced in the MNs' data traffic during a S/PGW relocation. For this, we investigate different performance measures for the MNs performing a handover with S/PGW relocation. In particular we study the latency of MNs' data packet delivery before and after handover ( $\S 5.6 .1$ ), CDFs of latency of the MNs' downlink data packets ( $\S 5.6 .2$ ), and packet loss in the redirected downlink data traffic (§ 5.6.3).

As a means of providing reliable performance results and hence insight into the system behavior, we provide mean results with $95 \%$ confidence interval by repeating the simulations for the same setting, using 10 random seeds. Note that due to partial overlap of the confidence intervals for different input values, they are presented for limited number of measurement points and graphs, for better presentation purpose.

\subsubsection{Average Latency of Data Packets Delivery Before and After Handover}

Fig. 5.4 presents for both approaches, the average latency of the data packets received by the moving MNs via the alternative downlink paths (before and after handover). The graphs clearly demonstrate that none of the proposed solutions have significant impact on the latency of the data packets redirected to the MNs after a handover. Slightly better result is observed for the scenario with the OpenFlow-Only switches, compared to the one with OpenFlow-Hybrid switches. This is due to the fact that, in the former solution only one action (Output) is added to the flow's set of actions in the switches. This accordingly imposes lower processing latency on the incoming flows, compared to the second approach, where two actions (Output and Set-Field) must be added to the switches.

The placement of the SDN Controller in the transport network can be critical, as it must receive within reasonable time the MN's mobility information from the MME in order to decide for the switches and setting up the traffic redirection paths. Therefore, we also studied the impact of it's position in the network architecture. Given that the MME uses the transport network to signal the SDN Controller, the performed experiments show that no significant impact 
$(\leq 3 \mathrm{~ms})$ is noted in the average latency after handover, where the distance between the SDN Controller and MME is changed from 1 to 7 hops.

In the simulation scenarios, the Egress OF switches are placed in the fixed positions with one hop distance to the S/PGWs. Positioning of the Ingress OF switch mainly depends on the topology (and size) of the transport network and should be as close as possible to the Internet (e.g., on the transport network's Internet PoPs (Point-of-Presences)).

Our experiments show that after handover, the average latency of data packet delivery has a little variation $(\leq 5 \mathrm{~ms})$, where the distance between the Ingress and Egress OF switches is changed from 1 to 7 hops. This accordingly may slightly affect the throughput of the proposed solutions.

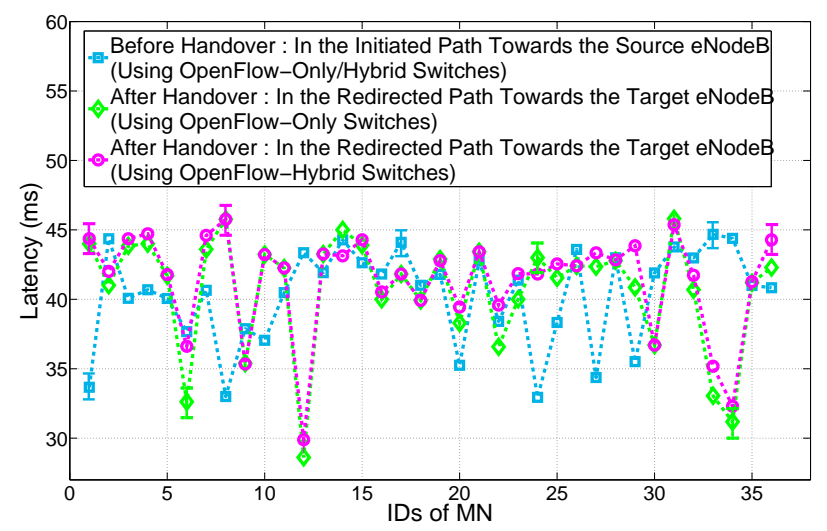

Figure 5.4: Average latency of the MNs' downlink data packets delivery before and after handover procedures.

\subsubsection{CDFs of Latency of the MNs' Downlink Data Pack- ets}

Fig. 5.5 presents the CDFs of latency of the first data packets delivered to the moving MNs after completion of a handover, using the proposed approaches. We select the first data packet of the MNs, as it is the most delayed packet after the handover procedure. This is because its delivery time is directly influenced by the time required to establish the traffic forwarding paths in the transport network. The results show that both approaches are fast enough to easily meet the maximum allowed latency of $150 \mathrm{~ms}$ [9] as a threshold for the VoIP application. It is also observed that the approach with the OpenFlow-Only switches outperforms the one with OpenFlow-Hybrid switches. As mentioned before, 
this is because in the former one less number of actions must be applied to the incoming flows during the traffic redirection procedure, compared to the second approach.

Fig. 5.5 also shows, for both approaches, the CDFs of latency of the data packets forwarded from the source eNodeB to the target eNodeB through the $\mathrm{X} 2$ path during the MNs' handover. Although X2 interface is a useful feature introduced in $3 \mathrm{GPP}$ standards to provide a seamless mobility to the MNs moving between the neighboring eNodBs, the graph shows that both proposed solutions significantly outperform the X2-path traffic redirection.

Therefore, in the case of using a mobility prediction mechanism e.g., [73] to provide information about the next visiting location of $\mathrm{MN}$ (e.g., target eNodeB), the traffic redirection paths in the SDN-based solutions can be set up a priori and hence avoid the usage of the X2 path data forwarding.

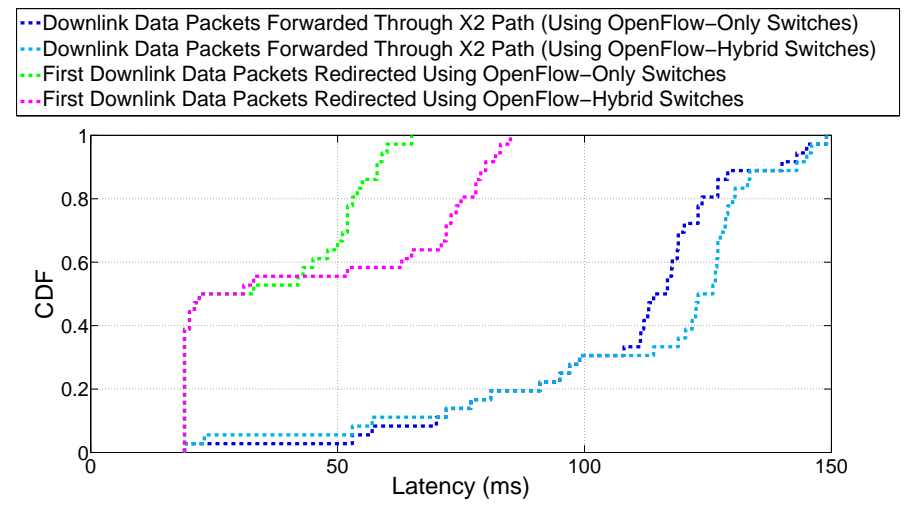

Figure 5.5: CDFs of latency of the MNs' downlink data packets via the X2 and proposed schemes traffic redirection paths.

\subsubsection{Packet Loss Ratio in the Redirected Downlink Data Traffic}

The X2 path is used to forward the MNs' downlink data during handover procedure from previous to the new eNodeB, and also after the handover when the traffic redirection paths of the SDN-based solutions are not yet set up. By using the X2 path for $10 \mathrm{~ms}$ after handover procedure (by setting Delete Session Timer $=10 \mathrm{~ms}$ in the MME [20]), no loss is present in the MNs' data packets. It implies that $10 \mathrm{~ms}$ is sufficient time, for both schemes, to establish the traffic forwarding paths in the transport network. 
Mobile network operators may not prefer to use the X2 path after the MNs' handover (setting Delete Session Timer $=0$ ). In this case, the moving MN's session is removed from the source $\mathrm{S} / \mathrm{PGW}$, once the $\mathrm{S} 1$ bearer is initiated at the target $\mathrm{S} / \mathrm{PGW}$. This may result in some packet loss for the MNs moving to a new $\mathrm{S} / \mathrm{PGW}$, see Fig. 5.6.

The results show that, the solution with OpenFlow-Only switches is more reliable than the one with OpenFlow-Hybrid switches. This is due to the fact that, the former scheme is faster than the second one in setting up the traffic redirection paths and the X2 path traffic forwarding takes shorter during the handover procedure. This implies that the solution with OpenFlow-Only switches provides a lower packet loss ratio while the X2 path is not even used during the the MNs' handover.

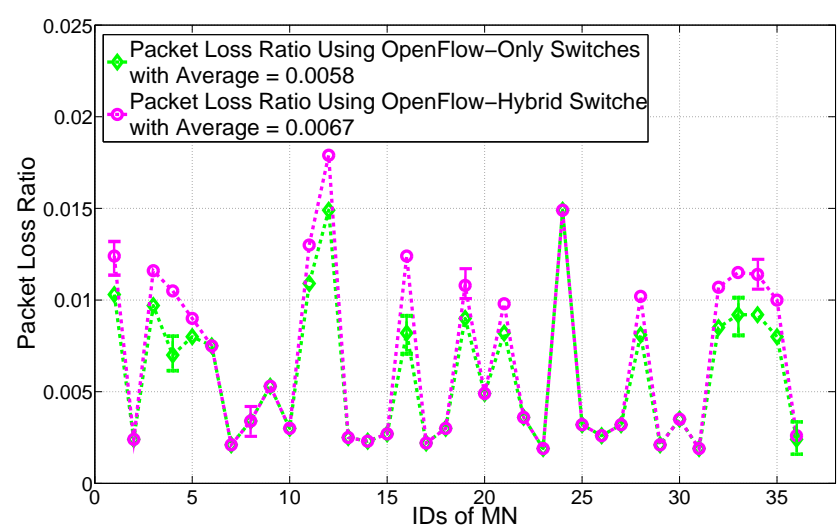

Figure 5.6: The MNs' downlink data packets loss ratio without using the X2-path traffic redirection.

\subsubsection{Control Messaging Overhead of the Proposed Solu- tions}

In the proposed approaches, several messages must be exchanged between the MME and SDN entities in order to set up a traffic redirection path (see Fig. 5.3). Assuming that only the messages from the SDN Controller towards the OpenFlow switches are sent through the transport network, Fig. 5.7 shows the overhead of the control messages of the proposed solutions on the network (while distance between the Ingress and Egress OF switches $=6$ hops), as a function of the number of MNs handed over to a new S/PGW. It is observed that the solution with OpenFlow-Hybrid switches outperforms the one with 
OpenFlow-Only switches and has lower impact on the network load. This is due the fact that, in the former solution the SDN Controller only makes the forwarding decision on the Ingress and Egress OpenFlow switches, placed at the edges of the transport network, and steering of traffic in the core part is performed by the conventional IP routing operation. This accordingly results in generating lower amount of control messages per $\mathrm{MN}$ in the network. In this figure, we also compare our approaches with the overhead impact of the existing centralized solutions (the GTP [21] and PMIP [20] protocols in the current LTE network), where a SGW relocation happens during the MNs handover.

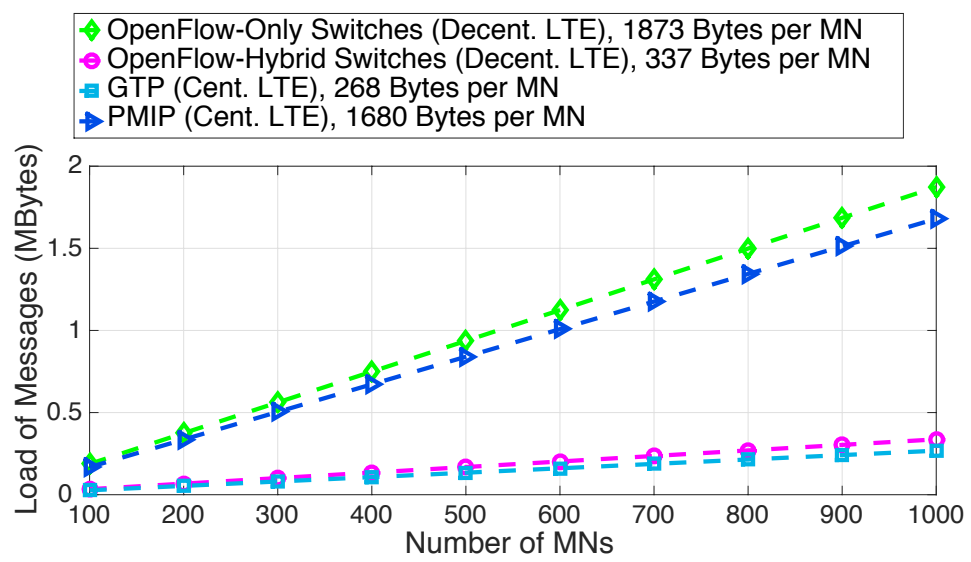

Figure 5.7: Overhead of the control messages.

For each approach, the graph shows the load of control messaging, in terms of the number of bytes traversed through the core network during the MNs' handover procedure. One may note that, as the number and size of the messages used for each $\mathrm{MN}$ is constant, the graphs grow linearly by increasing the number of MNs performing a hand over among the SGWs (S/PGWs).

\subsection{Concluding Remarks}

In this chapter, we have developed two novel approaches to support IP address and traffic continuity for the MN's active flow(s) after a handover with $\mathrm{S} / \mathrm{PGW}$ relocation in a LTE with decentralized network architecture. Our solutions are based on SDN, which is also a promising technology for the $5 \mathrm{G}$ mobile network. The proposed solutions are different based on using the OpenFlowonly or OpenFlow-hybrid switches for the implementation of the transport net- 
work. In the former approach, all the switches in the transport network must be controlled by the SDN Controller to set up traffic redirection towards the MN's current anchor points. In the latter scheme, only the Ingress and Egress switches need to be managed by the SDN Controller and the rest can be even replaced by the normal L3 routers. Therefore, implementation of the solution with OpenFlow-Hybrid switches is easily feasible with a minimal overhead and complexity. Detailed simulations show that both proposed solutions are fast enough in setting up the traffic redirection path considering the maximum allowed delay threshold for real-time applications (e.g., VoIP). The solution with OpenFlow-Only switches outperforms the one with OpenFlow-Hybrid switches in terms of the delivery latency and loss ratio for the downlink data packets, but provide higher overhead of the control messages on the network. 




\section{Transport Layer Approach, MPTCP-based Solution}

In this chapter, we benefit from the MPTCP protocol feature on supporting multiple IP addresses for a single data-stream and its break-before-make option to develop our last solution. The proposed solution can handel MNs' mobility in the decentralized LTE network without demanding an additional network interface and uses only one $3 G P P$ interface on MNs. MPTCP-based solution implements the MNs' mobility management functions in the transport layer of the end host entities, and keeps the network infrastructure intact. The publication related to this chapter is [70].

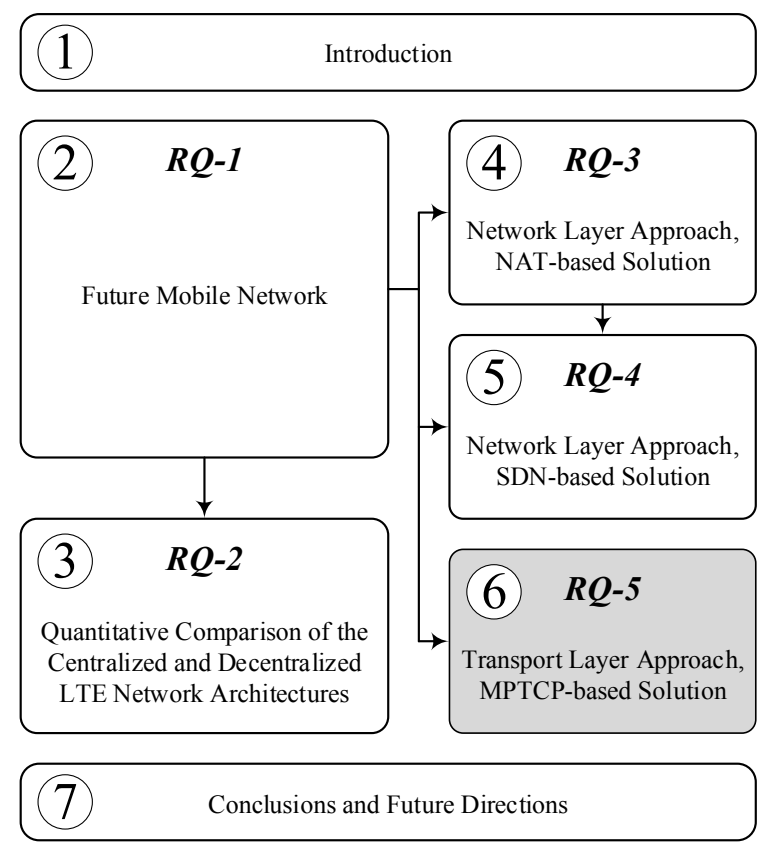


This chapter is organized as follows:

- Section 6.1 reviews briefly recent related work and states the contribution of this chapter.

- Section 6.2 describes the functional approach of the proposed solution.

- Section 6.3 presents the architecture, components, and control messages of the proposed solution.

- Section 6.4 describes the evaluation scenario and implementation of its components in the NS3-LENA simulation environment.

- Section 6.5 defines setting of the parameters in the simulation environment.

- Section 6.6 defines the performance metrics for the validation and presents and discusses the obtained results accordingly.

- Section 6.7 concludes this chapter. 


\subsection{Related Work}

In this section we briefly look at the recent related works and state how our work differs from the literature.

Benefiting from the multiple IP addresses support feature of the MPTCP, several approaches have been proposed aiming to e.g., improve bandwidth utilization, enhance traffic reliability, and smoother handover over different access networks. In this section we discuss some recent works on MPTCP-based mobility management and in particular focus on the novel aspects of our approach compared to the previous studies.

The work in [92] presents a mechanism relying on the cooperation of the MPTCP protocol and dynamic DNS. The proposed solution is based on the ID/Locator separation concept, where each MPTCP-enabled MN has a permanent IP address as ID, and several temporary IP addresses as locators. The IDs of MNs are used as the source and destination IP addresses (by the TCP applications), while the locators are used in the network layer for routing. During the traffic forwarding procedure, IDs of the data packets are replaced by locators, and for each MN the related mapping is stored in the DNS server. In order to support mobility functions upon moving to a new location, the MN updates its new locators on the DNS server. Next the CN, who has an initial TCP session with the MN gets the MN's ID and locators through the DNS and accordingly sets up a new MPTCP connection. In [83] an experimental study has been performed to evaluate the performance of MPTCP in handling an MN's handover from the $3 \mathrm{G}$ to WiFi network. The authors in [54] discuss a seamless TCP session continuity, where multiple paths are provided through independent access technologies such as cellular and fixed wire-line networks. To avoid MPTCP's inherent deployment issue at the end points, they proposed a lightweight MPTCP proxy to be integrated into the mobile devices, network routers or data centers to handle $\mathrm{TCP} \leftrightarrows \mathrm{MPTCP}$ translation via straightforward packet-header rewriting. In the context of LTE network, SIPTO is introduced by $3 \mathrm{GPP}$ [16] in order to selectively breakout some of the IP traffic closer to the MN. SIPTO allows the selected IP traffic to be directly routed within the access networks, while non-offloaded traffic is routed towards the LTE core network. In the framework of SIPTO, traffic session continuity is not supported, as the IP address of the MN has been changed due to mobility. In [39] a MPTCP-based solution has been proposed to maintain a single session active for the MN, changing its connection from one SIPTO entity to another. In [38] a baseline architecture has been proposed to develop a distributed mobility management scheme on a $3 \mathrm{GPP}$ flat network. In this research MPTCP is used to remove the chain of IP preservation of the current mobility management 
solutions, and instead to benefit from the available multiple paths to provide traffic session continuity to MNs.

The common assumption for all the previous works is the MN to be equipped with multiple access interfaces (e.g., WiFi, 3GPP, Wired), enabling it to set up multiple parallel connections for a single TCP session. The MN may activate the interfaces and accordingly may establish the subflows simultaneously (to improve, e.g., throughput or reliability) or one after the other (to handover among different wireless networks with heterogeneous access technologies) through the available channels towards a $\mathrm{CN}$.

Our work differs from the literature, in respect of the development of a new MPTCP-based scheme that enables a seamless TCP-based traffic forwarding and session continuity for the moving MNs in a decentralized LTE network system, while using only one $3 \mathrm{GPP}$ interface on the MNs. We detail on the functionality of the control and data planes, messaging, and architecture of the proposed approach to realize its integration in the new LTE architecture. We extend the NS3-LENA simulation environment to implement a LTE network with decentralized architecture, being integrated with the proposed solution and to evaluate the functionality and performance of the developed approach. To the best of our knowledge, we are the first exploiting the MPTCP in the context of mobility management in a decentralized LTE architecture.

\subsection{Approach}

As already stated in Chapter 2, during MNs' handover procedure with a S/PGW relocation in a decentralized LTE network, two layers of mobility support mechanism are needed in order to: (i) handle MNs' data traffic forwarding between two eNodeBs (inside the EPC network); (ii) to steer MNs' data packets between the source and target S/PGWs (outside the EPC network). We addressed the first step in $\S 2.4$ using the modified X2-based handover mechanism. In this section, we present the functional approach for a mechanism to handle the MN's traffic redirection above the EPC network (in the transport network). The proposed scheme relies on the MPTCP, originally proposed to improve network throughput, resilience in the case of path failure, load balancing as well as to enable multiple network access for multi-homed (interfaces) hosts. MPTCP is a set of TCP extensions [42], enabling transmission of a single TCP session through several subflows, with different IPs and/or ports. The packets belonging to various subflows can be transmitted via different available network paths towards the end point entities. The transport layer of MPTCP is splitted into application-oriented semantic layer (managing the application's end-to-end communication) and network-oriented Flow/End point layer (handling conges- 
tion control and endpoints identification), making it transparent to both the higher and lower layers [41], see Fig. 6.1.

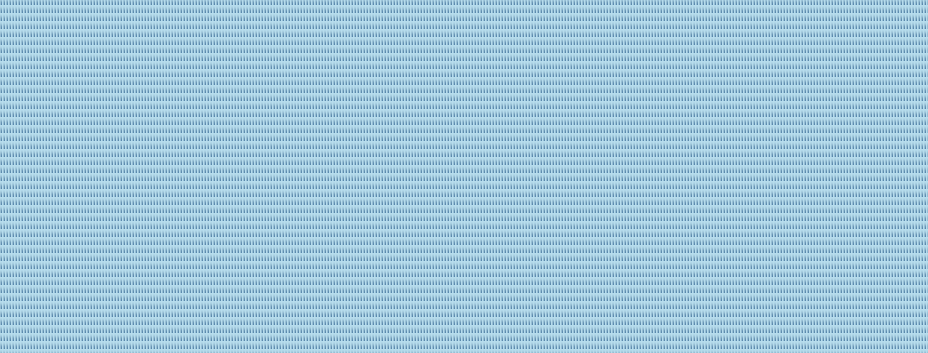

Figure 6.1: TCP and MPTCP protocol stacks.

MPTCP uses the standard TCP options [84] for signaling. In addition, the MPTCP options consist of a set of sub-types as listed in Table 6.1. This enables supporting multiple connections between the end hots for a single TCP session. As the public IP address or port number of the end point entities may change in the network (e.g., due to a NAT function), MPTCP assigns an 8-bits ID for each IP address. These IDs are not affected during the IP address modification and used by the end hosts to identify the subflows to be added to (or removed from) a particular connection.

Table 6.1: MPTCP options subtypes.

\begin{tabular}{|l|l|l|}
\hline Value & Symbol & Definition \\
\hline \hline 0x0 & $M P_{-} C A P A B L E$ & Multipath Capable \\
0x1 & $M P_{-} J O I N$ & Join Connection \\
0x2 & $D S S$ & Data Sequence Signal \\
0x3 & $A D D \_A D D R$ & Add Address \\
0x4 & $R E M O V E \_A D D R$ & Remove Address \\
0x5 & $M P_{-} P R I O$ & Change Subflow Priority \\
0x6 & $M P_{-} F A I L$ & Fallback \\
0x7 & $M P_{-} F A S T C L O S E$ & Fast Close \\
0x8-0xe & Unassigned & --- \\
0xf & Reserved for private use & --- \\
\hline
\end{tabular}

MPTCP supports three modes of operation [32]: 
- Full-handover mode, which refers to the regular MPTCP operation where all subflows are used simultaneously between two communicating hosts. The main objective of this mode is to obtain the best traffic rate for the users. This mode also provides the users a fast switching over different access technologies (e.g., WiFi and cellular networks).

- Backup mode, in this mode MPTCP initiates subflows over all the existing interfaces but uses only a subset of those for data transmission. The subflows specified by MP_PRIO option act as backup subflows, and are only used when the others are inoperative (e.g., due to a path failure or an invalid IP address). By this mode MPTCP can choose a premier path, for example it can use the available WiFi network and only switch to the cellular network when there is no WiFi connectivity

- Single-path mode, which is similar to the backup mode, except that at any moment only a single subflow is established and used for each MPTCP connection. When the previously used interface (or path) goes down, a new subflow over the other interface (available path) is established and used for data forwarding. This mode benefits from the break-before-make capability of MPTCP, to maintain the ongoing session active and continue data transmission without disturbing the application.

In the following section, we describe the architecture and functional procedure of our proposed solution in detail. It benefits from the MPTCP's single-path operation mode to steer the MN's traffic on the transport network, during the MN's handover with S/PGW relocation in a decentralized LTE network.

\subsection{Architecture}

This section details the architecture, components, and control messages of our MPTCP-based solution developed to manage seamless traffic session continuity for the MNs moving among the distributed S/PGWs in a LTE network with decentralized architecture.

In a decentralized network architecture, the MN's IP address is anchored at the distributed anchor points. Hence, when an MN moves from one anchor point to another it receives a new IP address on the new network attachment. In this situation, the MN's established IP based connection will not survive as it relies on constant endpoints IP addresses. Therefore, the MN's initial connection must be somehow protected upon obtaining a new IP address. For this, a supplementary mechanism must be applied. Through such a mechanism either the relevant endpoint updates the corresponding address or continues with the same addresses, using IP address translation or tunneling techniques. 


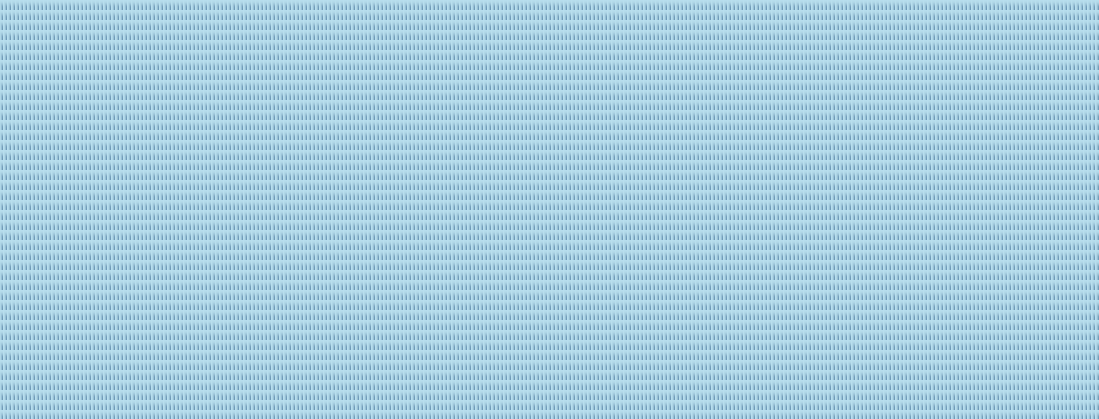

(a) Before Handover
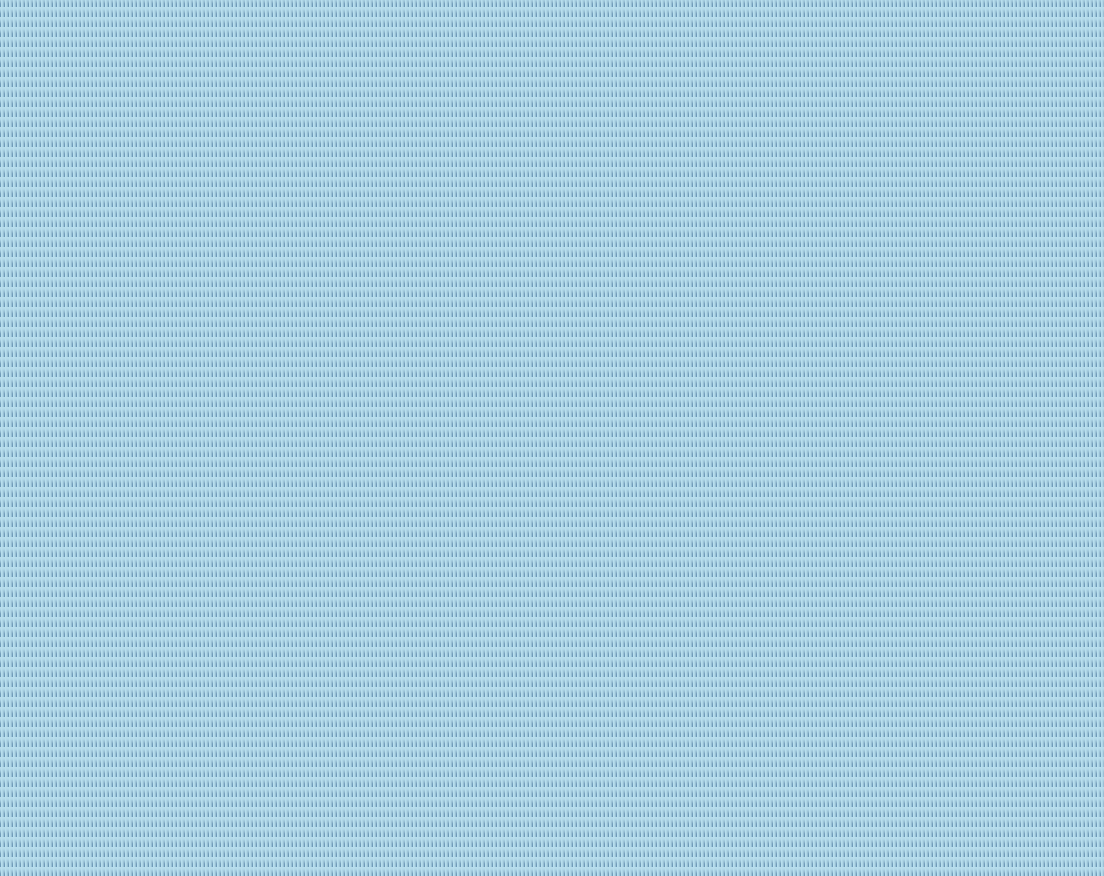

SubFlow-2 T-eNodeB

IP address:

B.Y.Y.1

(c) After Handover

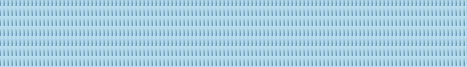

- Plain IP GTP tunnel ----- Standard Control Line

Figure 6.2: MPTCP to support the MN's traffic redirection and mobility. 
Our new approach handles the MN's mobility on the transport layer with the minimal impact on the network deployment. We benefit from the MPTCP protocol feature on supporting multiple IP addresses for a single data-stream and its break-before-make option to preserve the MN's ongoing traffic continuity while moving as described above.

Fig. 6.2 shows the architecture of the proposed approach to manage the above-mentioned aspects in a decentralized LTE network. Given that the MN and $\mathrm{CN}$ are MPTCP-aware entities, in this architecture the subflows are added to/removed from the MN's active traffic via the available/inaccessible paths to support seamless session continuity upon handover between two eNodeBs served by different S/PGWs.

\subsubsection{Description of Control Messages and Flow Diagrams}

Fig. 6.3 shows the flow of control messages of the proposed scheme. In order to set up/remove the alternative downlink paths in the transport network towards the MN's current location, several messages are exchanged between the EPC and MPTCP approach entities. This section describes briefly the messages used for these procedures.

\subsubsection{During PDN Connection Procedure}

Assume that a MPTCP-enabled MN attaches to the source eNodeB to inquire some data from the CN (see Fig. 6.2(a)). In the decentralized LTE network, as discussed in $\S 2.3$, the MN's attach procedure is similar to the one in the current LTE system ( $\S$ 5.3.2.1 of [20]), by co-locating the SGW and PGW as S/PGW. After receiving the MN's PDN Connection Request message by the MME, in order to establish the initial path for the MN's downlink traffic (SubFlow-1), the following messages are exchanged between the EPC and MPTCP approach entities (see top-part of Fig. 6.3):

(1) the MME sends a Create Session Request to the source S/PGW and asks to establish a new bearer to the attached MN. In the reply, the source $\mathrm{S} / \mathrm{PGW}$ sends the Create Session Response message and notifies the MME about the assigned IP address to the MN (e.g., A.X.X.1).

(2) the MN establishes a connection to the CN by sending a $S Y N$ packet through the source $\mathrm{S} / \mathrm{PGW}$. This packet includes the MP_CAPABLE option, specifying that the MN supports MPTCP protocol and wishes to initiate a MPTCP-based connection. 


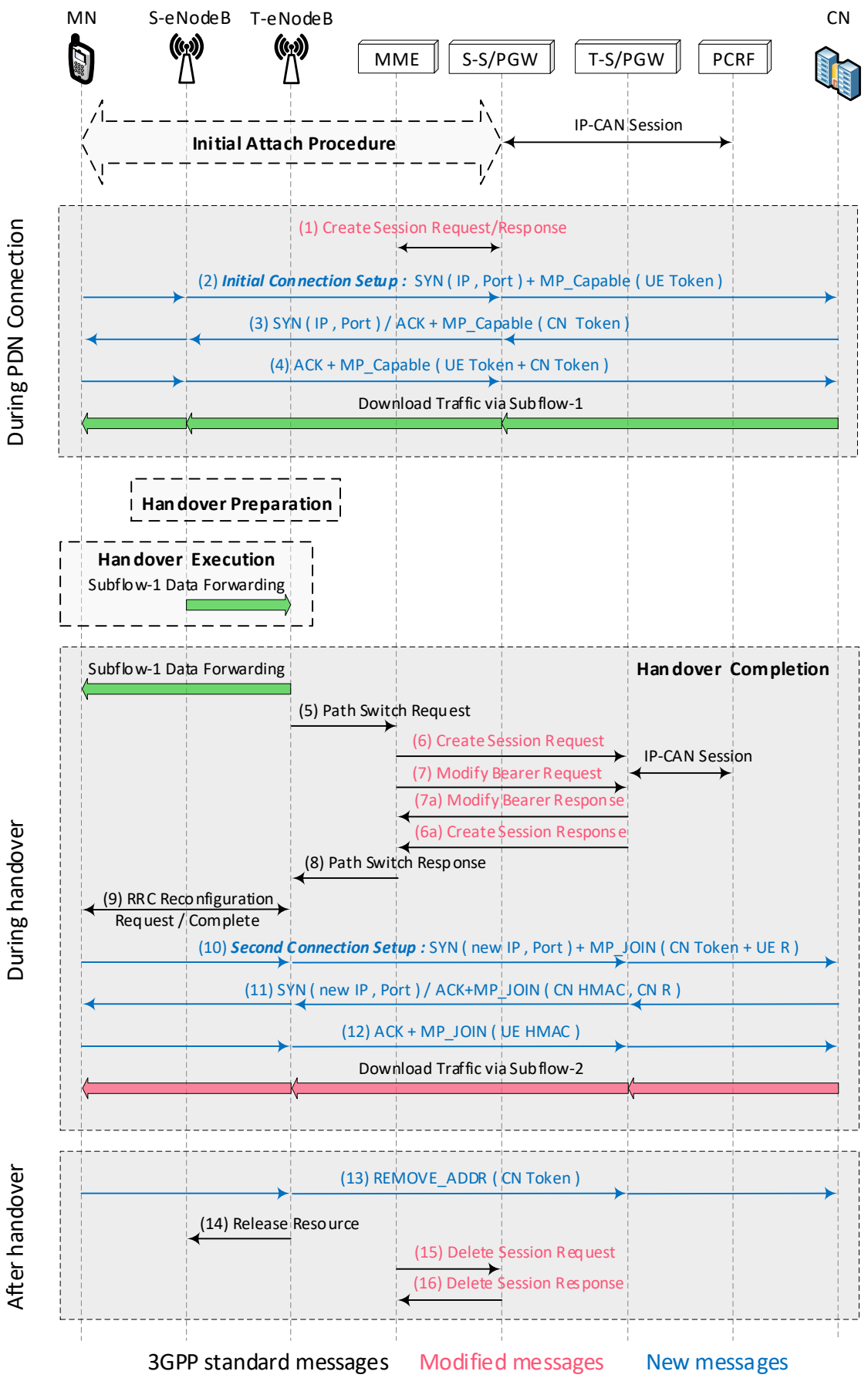

Figure 6.3: Control messages flow diagram of the MPTCP-based solution. 
(3) if the $\mathrm{CN}$ is also a MPTCP capable entity, it responses to $\mathrm{MN}$ by sending a $S Y N / A C K$ packet, containing the $M P_{-} C A P A B L E$ option as well. The $M P_{-} C A P A B L E$ option, exchanged between the $\mathrm{MN}$ and $\mathrm{CN}$, carries the unique identifiers for the $\mathrm{MN}$ and $\mathrm{CN}$, entitled $M N$ Token and $C N$ Token, respectively.

(4) the MN completes the three-way handshake with an $A C K$ packet which also carries a $M P_{-} C A P A B L E$ option (with $M N$ Token $+C N$ Token). These tokens are used by the endpoints to identify the connection and to authenticate each other, when a new subflow is added to the active connection.

Upon a successful MPTCP connection establishment between the CN and MN, the MN's downlink data is transmitted through the transport network towards the source S/PGW, via Subflow-1 using IP addresses: C.Z.Z.1 $\rightarrow$ A.X.X.1 (the green solid-line between the $\mathrm{CN}$ and source S/PGW in Fig. 6.2(a)).

\subsubsection{During Handover Procedure}

In our solution we used the X2-based handover procedure, already specified in $\S 5.5 .1 .1 .3$ of [20] for the MN performing a handover with SGW relocation, as the baseline to define a new model of MN's handover between different S/PGWs, see $\S 2.4$. In the X2-based handover procedure, the MN's downlink traffic forwarding between two eNodeBs is fulfilled without the EPC network involvement, and the related control messages on the Handover Preparation phase are directly exchanged between the source and target eNodeBs. Thereby, during the Handover Executing phase the source eNodeB forwards the MN's downlink data, received via Subflow-1 from the source S/PGW, towards the target eNodeB (see Fig. 6.3). The Handover Preparation and Executing phases are based on the LTE's standard specified in $\S$ 19.2.2.5.1 of [18]. Once the handover has been executed successfully (Handover Completion phase) and the MN is connected to the target eNodeB, the buffered data in the target eNodeB is delivered to the MN (the green solid-line between two eNodeBs in Fig. 6.2(b)).

In order to set up a second path for the MN's newly established downlink traffic (SubFlow-2), following messages are exchanged between the EPC and MPTCP approach entities (see middle-part of Fig. 6.3):

(5) in the Handover Completion phase, the target eNodeB sends a Path Switch Request message to inform the MME about the newly connected MN and its request for a new PDN connectivity. 
(6, 6a \& 7, 7a) the MME exchanges the Create Session Request/Response and Modify Bearer Request/Response messages with the target S/PGW and asks to create a new bearer for the handed over MN. The target S/PGW creates a new entry in its EPS bearer table among other required information [23], allowing it to route MN's data between the E-UTRAN and the external network. After the bearer allocation, the target S/PGW allocates an IP address (e.g., B.Y.Y.1) from its address pool to the MN.

(8) the MME responds back to the target eNodeB with the Path Switch Response message, including among other information (§5.10.2 of [23]) IDs of the established EPS bearers, IP address of the target S/PGW and the newly assigned IP address of the MN.

(9) the target eNodeB exchanges the RRC Reconfiguration Request/Complete messages with the MN and informs it about establishment of the new PDN connection and the new IP address allocated by the target S/PGW.

(10) the MN uses its new IP address and initiates a new connection by sending a $S Y N$ packet through the target $\mathrm{S} / \mathrm{PGW}$ to the $\mathrm{CN}$. The new subflow may be opened either over a new IP address of the CN (e.g., C.Z.Z.2) or the same IP address used for the previous subflow (C.Z.Z.1) with a different CN's TCP port. The $S Y N$ packet carries also the MP_JOIN option, specifying that $\mathrm{MN}$ wishes to add a new subflow on a particular connection. The $C N$ Token in the MP_JOIN option, is used as an identifier to determine to which MPTCP connection the new subflow must be associated.

(11 \& 12) thereupon, the CN and MN reply each other with the $S Y N / A C K$ and $A C K$ packets, respectively, and complete the three-way handshake procedure for the new connection. During connection establishment of the second subflow, the endpoints exchange also several authentication keys (HMAC and $R$ [42]). This allows the $\mathrm{CN}$ to verify that the $\mathrm{MN}$ is the same as the one that set up the initial connection (for subflow-1).

By setting up the new connection, the CN starts to transmit the MN's downlink data towards the target $\mathrm{S} / \mathrm{PGW}$, via the newly initiated subflow (i.e., Subflow-2), using IP address: C.Z.Z.2 $\rightarrow$ B.Y.Y.1 (the red solid-line between the $\mathrm{CN}$ and target $\mathrm{S} / \mathrm{PGW}$ in Fig. 6.2(c)). 


\subsubsection{After Handover Procedure}

After being detached from the source eNodeB, in order to remove the previously established path in the transport network, the following messages are exchanged between the EPC and MPTCB-based approaches entities (see bottom-part of Fig. 6.3):

(13) the MN uses the newly established connection through the target eNodeB and sends a REMOVE_ADDR option to inform the CN about the lost connection. This option specifies the IP address (A.X.X.1) being unavailable for the $\mathrm{MN}$ after movement. Upon reception of this message, the $\mathrm{CN}$ terminates the TCP subflow associated with this IP address (i.e., SubFlow-1).

(14) the target eNodeB asks the source eNodeB to release the resources by sending a Release Resource message.

(15 \& 16) MME and source S/PGW exchange the Delete Session Request/Response messages to remove the corresponding bearers related information.

In both procedures (before and after handover), upon arrival of the data packets at the corresponding S/PGW, they are processed and encapsulated into a GTP tunnel and then forwarded to the relevant eNodeB to be delivered to the MN via the LTE air interface.

\subsection{Setup of Simulation Study}

This section presents the evaluation scenario and implementation of the components in the simulation environment. We set up the simulation environment as realistic as possible to get reliable results, as described below.

\subsubsection{Evaluation Scenario}

The logical network topology of the evaluation scenario is the one shown in Fig. 6.2. During 20 seconds simulation, $120 \mathrm{MNs}$ attach to each eNodeB and generate the E-UTRAN traffic according to Table 6.2 . $30 \%$ of the MNs of the source eNodeB (MPTCP-enabled) run a TCP-based application (with 500 bytes packet size and 10 packets/second traffic stream) and move to the target eNodeB at different times between 10.74 and 12.51 seconds after starting of the simulation time. The modified X2-based mechanism (within the EPC) and the 
MPTCP-based solution (within the transport network) handle forwarding of the MNs' downlink traffic towards the target position.

\subsubsection{Implementation of Scenario in the NS3-LENA}

We extended the NS3-LENA simulation environment to implement the required components and to evaluate the functionality and performance of the proposed solution. The extensions are described below. The link of the source code for implementation of the all modules can be found in appendix B.

\subsubsection{Decentralized LTE Network}

This implementation is similar to the one described in $\S 4.4 .2 .1$. The only difference is on the 4.4.2.1.(ii) part, where we modified the Create Session Request/Response and Modify Bearer Request/Response messages, exchanged between the MME and the target S/PGW to create a new DL-TFT in the target S/PGW, after the Handover Completion phase. These changes realize the implementation of an EPS bearer and allocation of an IP address for the moving MN. Accordingly, this enables establishing a new data plane for the MN through the target S/PGW after its handover.

\subsubsection{Transport Network}

The implementation of the transport network is the same as the one described in $\S 4.4 .2 .2$.

\subsubsection{MPTCP Function}

We extend the MPTCP module, already developed in [5, 47], to integrate the MPTCP protocol functions in the discussed decentralized LTE network architecture. Our modifications are based on the requirements of the solution presented in this chapter (see Fig. 6.3) .

\subsubsection{MNs' Movement}

The implementation of MNs' movement is the same as the one described in $\S 4.4 .2 .5$.

\subsection{Simulation Parameters}

This section presents the setting of parameters in the simulation environment. 


\subsubsection{E-UTRAN Setting}

The configuration of the parameters for E-UTRAN is the same as the one defined in $\S$ 4.5.1.

\subsubsection{E-UTRAN Traffic}

We used the traffic mix model (see Table 6.2) specified in [60] to generate the RAN traffic. In the source eNodeB, a TCP-based traffic is selected as the one generated by the moving MPTCP-enabled MNs. Other types of traffic are used to generate the RAN background traffic by the stationary MNs attached to the both eNodeBs. Only the moving MNs' traffic is forwarded to the Internet and the rest is dropped at the eNodeBs.

Table 6.2: The E-UTRAN traffic model.

\begin{tabular}{|c|c|c|}
\hline Application & Traffic & Percentage of $\mathrm{MN}$ \\
\hline $\mathrm{TCP}^{*}$ & Interactive & $30 \%$ \\
\hline VoIP $^{* *}$ & Real-time & $30 \%$ \\
\hline FTP & Best effort & $10 \%$ \\
\hline HTTP & Interactive & $20 \%$ \\
\hline Video & Streaming & $20 \%$ \\
\hline Gaming & Interactive real-time & $20 \%$ \\
\hline
\end{tabular}

*) In the source eNodeB, **) In the target $e N o d e B$

\subsubsection{EPC and Transport Network Setting}

The configuration of the parameters for EPC and transport network is the same as the one defined in $\S 4.5 .3$.

\subsubsection{The EPC and Transport Networks Traffic}

The configuration of the parameters for this section is the same as the one defined in $\S 4.5 .4$.

\subsection{Performance Evaluation}

In this section, using the simulations specified above, we evaluate the seamlessness of the proposed mobility support approach. That is, if no significant delay and packet loss are introduced in the MNs' data traffic during a S/PGW relocation. For this, we consider several performance measures for the MNs 
performing a handover with $\mathrm{S} / \mathrm{PGW}$ relocation. In particular we study the latency of MNs' data packet delivery before and after handover ( $\S 6.6 .1)$, CDFs of latency of the MNs' downlink data packets ( $\S 6.6 .2$ ), and packet loss in the redirected downlink data traffic $(\S 6.6 .3)$.

As a means of providing reliable performance results and hence insight into the system behavior, we provide mean results with $95 \%$ confidence interval by repeating the simulations for the same setting, using 10 random seeds. Note that due to partial overlap of the confidence intervals for different input values, they are presented for limited number of measurement points and graphs, for better presentation purpose.

\subsubsection{Average Latency of Data Packet Delivery Before and After Handover}

Fig. 6.4 presents, the average latency of the data packets received by the moving MNs, using subflow-1\&2 via the alternative downlink paths (before and after handover). The graph clearly demonstrates that the proposed solution has no significant impact on the latency of the data packets, redirected to the MNs after a handover.

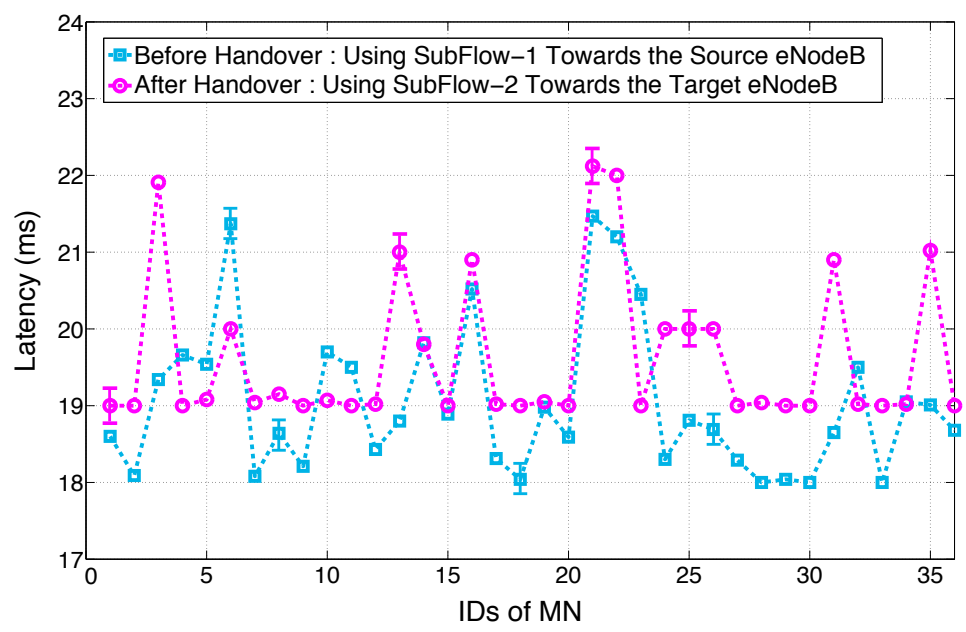

Figure 6.4: Average latency of the MNs' downlink data packets delivery before and after handover procedures.

Typically, the placement of the CN mainly depends on the topology (and size) of the transport network. Therefore, we also studied the impact of it's position in the network architecture. The performed experiments show that the 
average latency of data packet delivery has a little variation $(\leq 8 \mathrm{~ms})$, where the distance between the $\mathrm{CN}$ and the S/PGWs is changed from 1 to 7 hops. This accordingly may slightly effect the throughput of the proposed solution.

\subsubsection{CDFs of Latency of the MNs' Downlink Data Pack- ets}

Fig. 6.5 presents the CDFs of latency of the first data packets delivered to the moving MNs through the source $\mathrm{S} / \mathrm{PGW}$ (upon PDN connection procedure) and target S/PGW (after completion of a handover), using the alternative paths established by the MPTCP-based mobility support approach. We choose the first data packet of the MNs, as it is the most delayed packet during the delivery procedure. This is because its delivery time is directly influenced by the time required to build the connections (over the $\mathrm{S} / \mathrm{PGWs}$ ), and accordingly to set up the subflows and traffic forwarding paths in the transport network. The obtained results show that the establishment time for the second connection (subflow-2, using $M P_{-} J O I N$ option) is more than the time required to set up the initial connection (subflow-1, using $M P_{-} C A P$ option), but still is low enough to meet even the maximum acceptable latency for the real time application (e.g., 250 ms for the VoIP [9]).

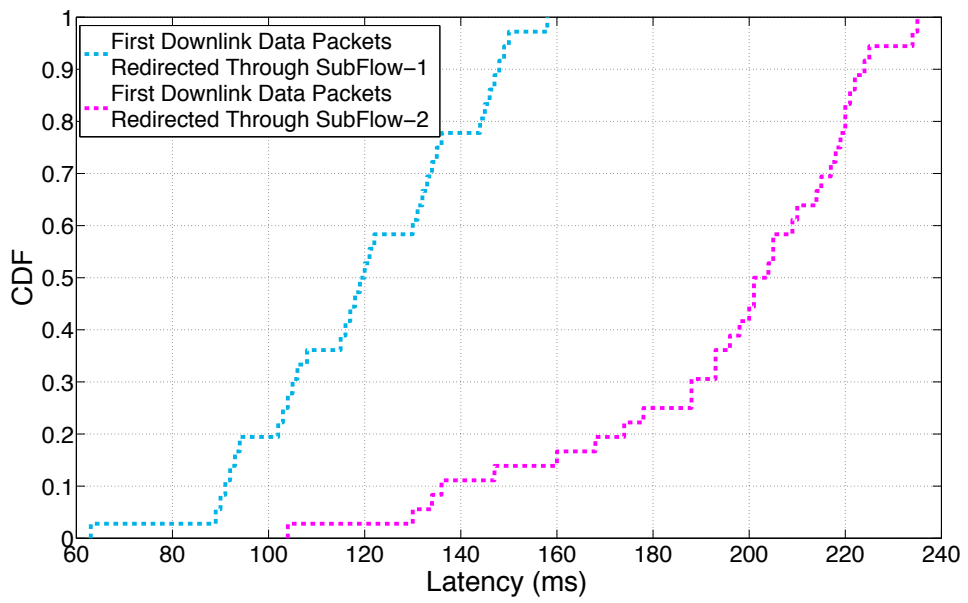

Figure 6.5: CDFs of latency of the MNs' first downlink data packets via subflow-1\&2. 


\subsubsection{Packet Loss in the Redirected Downlink Data Traffic}

The X2 path is used to forward the MNs' downlink data during Handover Execution, and also Handover Completion when the second subflow of the MPTCP-based solution is not yet set up. In the latter case, provided that the MN's initial session has not been deleted yet by the MME [20], the downlink packets are still being received at the source eNodB and delivered to the target one via the X2 path. By using the X2 path (for $\leq 200 \mathrm{~ms}$ ) after handover procedure, no loss is present in the MNs' data packets. It implies that $200 \mathrm{~ms}$ is sufficient time to establish the second subflow and to set up the new path in the transport network for forwarding the MN's active traffic to target position.

Mobile network operators may not prefer to use the X2 path traffic forwarding during the MN's handover. In this case, the moving MN's session is removed from the source $\mathrm{S} / \mathrm{PGW}$, upon the $\mathrm{S} 1$ bearer is initiated at the target $\mathrm{S} / \mathrm{PGW}$. This may result in some packet loss for the MNs moving to a new $\mathrm{S} / \mathrm{PGW}$. However, the experiments show that in the latter case (without using $\mathrm{X} 2$ interface) the MPTCP is able to quickly detect the failures and retransmits the MNs' unacknowledged data packets over the second active subflow. This accordingly may impose a small delay on the application due to the MPTCP's packet loss recovery process. Fig. 6.6 shows the packets lost (during handover) and the MPTCP retransmission events (after handover) for some of the MNs as examples.

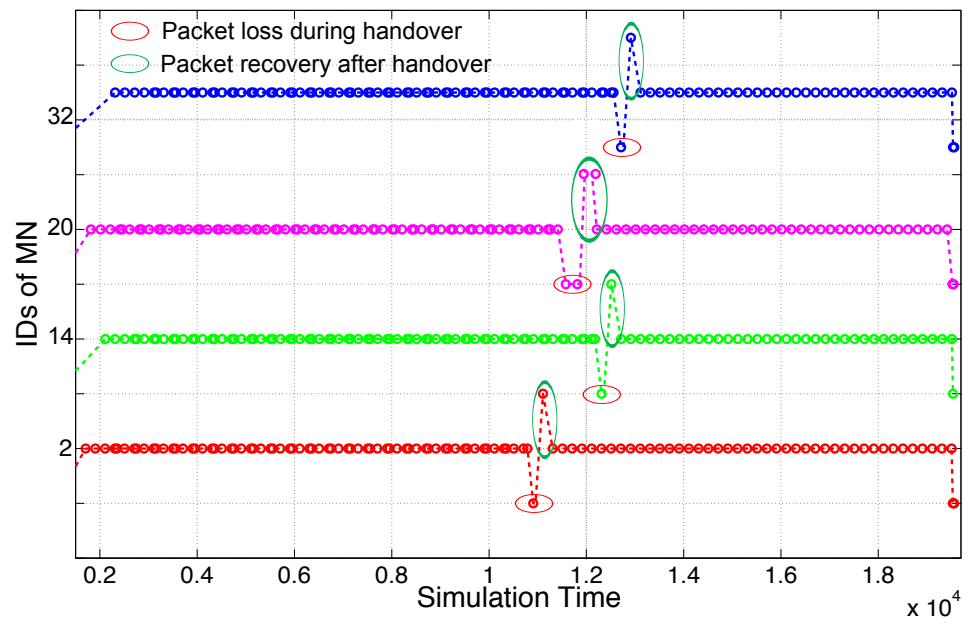

Figure 6.6: The MNs' downlink data packets loss without using the X2 interface and MPTCP loss recovery after handover. 


\subsubsection{Control Messaging Overhead of the Proposed Solu- tion}

In the proposed approach, several messages must be exchanged between the $\mathrm{MN}$ and $\mathrm{CN}$ entities (see Fig. 6.3) in order to set up the second subflow's traffic redirection path. Fig. 6.7 shows the overhead of the control messages of the proposed solution on the network as a function of the number of MNs handed over to a new $\mathrm{S} / \mathrm{PGW}$. It is observed that the proposed approach has negligible impact on the network load.

In this figure, we also compare our approach with the overhead impact of the existing centralized solutions (the GTP [21] and PMIP [20] protocols in the current LTE network), where a SGW relocation happens during the MNs handover.

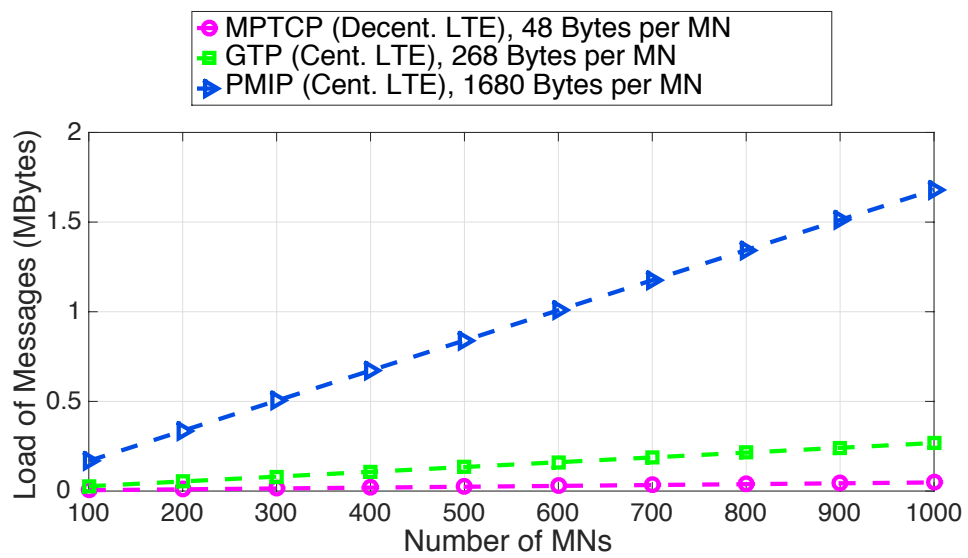

Figure 6.7: Overhead of the control messages.

For each approach, the graph shows the load of control messaging, in terms of the number of bytes traversed through the core network during the MNs' handover procedure. One may note that, as the number and size of the messages used for each $\mathrm{MN}$ is constant, the graphs grow linearly by increasing the number of MNs performing a hand over among the SGWs (S/PGWs).

\subsection{Concluding Remarks}

In this chapter, we have developed a novel scheme to support seamless traffic continuity for an MN's TCP session kept active after a handover with S/PGW 
relocation in a LTE network with decentralized architecture. Our solution is based on the MPTCP, which is also a promising protocol to be used in the $5 \mathrm{G}$ mobile network. The proposed solution can handle the MN's mobility without demanding an additional interface on the $\mathrm{MN}$ in a resource-efficient manner, with the cost of only four extra small size control messages (3-way TCP handshake \& Remove address) upon a handover. As only the MNs and the corresponding CNs need to be MPTCP-aware entities, implementation of the solution is feasible with a minimal overhead and complexity. Detailed simulations show that the proposed approach is fast enough in setting up the second connection and traffic redirection path, even when considering the maximum acceptable latency for a real-time application. Furthermore, it is also observed that the developed solution significantly outperforms the mobility support mechanisms of the current LTE in terms of the control plane overhead. Benefiting from the packet loss recovery mechanism of the MPTCP, the usage of X2-based data forwarding during the MN's handover can be avoided, with imposing a small delay on the application. 



\section{Conclusions and Directions for Future Research}

In this last chapter, we set out the main findings and contributions of our research. Furthermore, we present directions for future research.

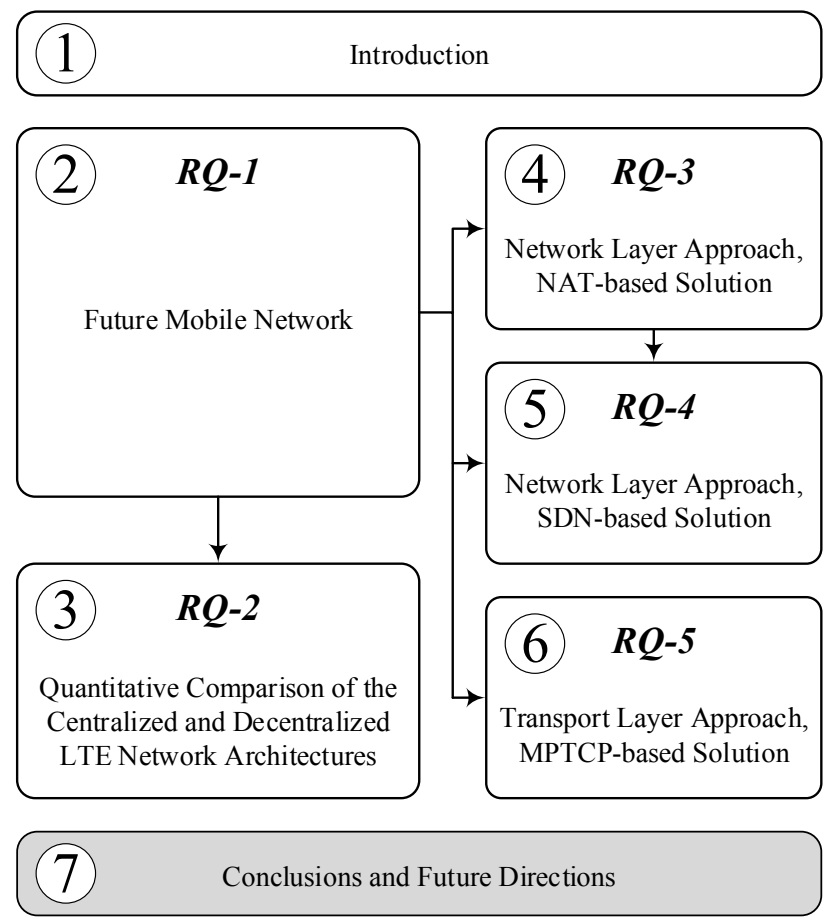


This chapter is organized as follows:

- Section 7.1 sets out the main conclusions of this thesis according to respective research questions.

- Section 7.2 summarizes the contributions of this thesis per chapter.

- Section 7.3 discusses directions for further research. 


\subsection{Conclusions}

In this section we present the main findings of this thesis according to the research questions as defined in Chapter 1.

As discussed in $\S 1.1$, traffic offloading in the proximity of the access network can effectively mitigate the traffic overhead on the core network. This leads to a decentralized network architecture, which enables to cope with the ever-growing demands on mobile networks.

Therefore, our RQ-1 was defined as:

[...] How can the current LTE network architecture be modified to realize a decentralized LTE system?

We addressed the RQ-1 in Chapter 2. We proposed an approach to decentralize the LTE network architecture, in which the S/PGWs, covering both the SGW and PGW functions, are distributed to the edge networks to handle the MNs' connection and mobility functions locally.

In the existing LTE system, IP traffic continuity is not supported when an MN changes its PGW. Following a decentralized LTE architecture, the MNs are expected to move often among the S/PGWs. To address the anchor points relocation issues in the LTE's new architecture, we modified the current standard LTE's X2-based handover procedure with SGW relocation, to be used to handle IP traffic forwarding and session continuity for the MNs handed over between different $\mathrm{S} / \mathrm{PGWs}$, see $\S 2.4$. This allows only to support MNs' mobility inside the EPC of the decentralized LTE network. In order to fulfill a complete procedure of mobility management, additional mechanisms are needed to enable the MNs' data traffic forwarding between the source and target S/PGWs above the EPC network. For this, we developed the three solutions discussed in Chapter 4, 5 and 6 .

Although, decentralization of the network architecture would be a promising approach to reduce the traffic load on core network entities, the gain of this approach on the efficiency of LTE network was an open question.

Therefore, we defined the RQ-2 as:

[...] To which extent can decentralization of the network architecture improve efficiency and scalability of the LTE system?

In Chapter 3, we aimed to quantify the impact of the number of connected devices to the network, on various performance and scalability metrics, for the 
current (centralized) and decentralized LTE network architectures. We carried out an extensive study comprising simulation and analytical models, to analyze and compare the performance of both architectures. In particular, we measured the attached MNs' data traffic and mobility related messages load, as well as the latency and cost for the initial attachment, handover, and data packet delivery procedures for both architectures, using GTP and PMIP protocols. Considering of the specified scenarios and parameters, Table 7.1 summarizes the gain of decentralization of the LTE network. In this table, the percentile values represent the decrease in the Growth Rate (GR) (of exponentially growing parameters) or Increasing Slope (IS) (of lineally growing parameters) of the parameters, and the scalar values show the decrease by ratio in the initial value of the parameters ( $Y_{100}$ centralizec $/ Y_{100}$ decentralizec).

Furthermore, in the decentralized architecture, the results show that the load of the root router (see Fig. 3.6) is around 11 times less than the centralized one.

Table 7.1: Possible gain after moving to a decentralized LTE architecture for the scenarios considered in Chapter 3.

\begin{tabular}{|l|l|l|l|l|}
\hline & GTP & GTP & PMIP & PMIP \\
\hline \hline Latency of initial attachment procedure & 44.35 & $15 \%$ & 7.63 & $6 \%$ \\
\hline Latency of handover procedure & 61.83 & $15 \%$ & 2.94 & $6 \%$ \\
\hline Latency of data packet delivery & 11.54 & $11 \%$ & 5.83 & $8 \%$ \\
\hline Processing cost of initial attachment procedure & 8.90 & $11.7 \%$ & 3.52 & $3.2 \%$ \\
\hline Processing cost of handover procedure & 12.31 & $12.2 \%$ & 2.30 & $2.1 \%$ \\
\hline Processing cost of data packet delivery & 6.74 & $9.4 \%$ & 3.13 & $1.1 \%$ \\
\hline
\end{tabular}

Exponentially growing parameters (the latency metrics, see Fig. 3.8a, 3.8c and 3.8e) in general are denoted by: $Y_{n}=Y_{n_{0}} \times(1+G R)^{n}$;

Linearly growing parameters (the processing cost metrics, see Fig. 3.8b, 3.8d and 3.8f) in general are denoted by: $Y_{n}=Y_{n_{0}}+I S \times n$,

Where $Y_{n}$ represents the performance metric corresponding to $n$ number of $\mathrm{MNs}$ and $n_{0}=100$, see Fig. 3.8.

We already explained in Chapter 2 that, the LTE network with decentralized architecture needs two layers of mobility management to handle MN's handover during a S/PGW relocation: (i) within the EPC network (we discussed it in 
$\S 2.4$ ); and (ii) above the EPC network. For the MN's mobility issue above the EPC we investigated several approaches, leading to the RQ-3, 4 and 5.

In this respect, we defined the RQ-3 as:

\section{[...] How can current network layer technologies such as NAT be applied to implement the MN's mobility related functions in a decentralized LTE network architecture?}

Our approach, discussed in Chapter 4, was based on NAT technique, which is widely used in the current Internet. The proposed solution relies on the Identifier-Locator split concept. The Identifier refers to the IP address of MN's flow(s) kept active after a handover (allocated by source S/PGW), and the Locator refers to the IP address used to forward downlink traffic to the MN's new position (assigned by target S/PGW). We introduced two NAT routers at the edges of the transport network to carry out the correct translation of the Identifier $\rightleftharpoons$ Locator addresses to redirect the ongoing flow(s) to the MN's new position. Deploying the NAT routers only at the edges of the transport network makes this solution to be deployable at a large scale with a minimal complexity.

Extensive validation experiments, using the NS3-LENA simulation environment, proved that the developed solution is fast enough in setting up the traffic redirection path and readily meets the latency requirement, considering the maximum allowed delay threshold for real-time applications (e.g., VoIP).

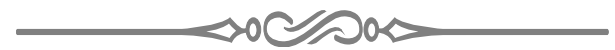

The growing interest from mobile networks on SDN motivated us to investigate also the feasibility of this technology on handling the MNs' mobility above the EPC network.

For this, we defined the RQ-4 as:

\section{[...] How can future network layer technologies such as SDN be applied to implement the MN's mobility related functions in a decentralized LTE network architecture?}

In Chapter 5, we exploited the potential of SDN on decoupling of control plane and data plane, and developed two solutions to handle MNs' mobility between S/PGWs. The proposed solutions differ in the sense that they use the OpenFlow-hybrid or OpenFlow-only switches for the implementation. The first approach is based on the modification of the headers of the flow-specific packets on the switches. In this solution, only the Ingress and Egress switches of the transport network must be OpenFlow-enabled switches, see Fig. 5.2. Hence, its integration with the current L3-based network, is feasible with a minimal 
complexity. The approach with OpenFlow-only switches relies on updating the flow's table of the switches, without any IP address translation or modification in the flow's packets. This solution, is more suited for the networks that are fully implemented with the SDN-based switches.

The validation experiments on the NS3-LENA simulation environment showed that both solutions perform fast enough in setting up the traffic redirection path considering the maximum allowed delay threshold for real-time applications. The solution with OpenFlow-Only switches outperforms the one with OpenFlow-Hybrid switches in terms of the delivery latency and loss ratio for the downlink data packets, but provides higher overhead of the control messages on the network.

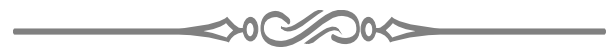

Given the facts that: (i) generally, the transport layer-based mobility management solutions have a minimal impact on the network infrastructure for implementation; and (ii) TCP is the most widely used protocol in todays Internet, we motivated to study the capability of MPTCP on handling the MN's mobility issues above the EPC network.

Therefore, we defined our RQ-5 as:

[...] How can transport layer technologies such as MPTCP be applied to implement the MN's mobility related functions in a decentralized LTE network architecture?

In Chapter 6, we gained from the MPTCP protocol feature on supporting multiple IP addresses for a single data-stream and its break-before-make option to develop our last approach. The proposed solution can preserve the MN's IP traffic session continuity during its movement between S/PGWs, without demanding an additional network interface (using only one 3GPP interface on MNs). Supporting the MPTCP protocol by most of the already existing smartdevices scattered over the network, make this solution easier to be applied in a large scale in today's mobile networks.

The validation experiments on the NS3-LENA simulation environment demonstrated that the developed solution is fast enough in establishing the new connection after MNs' handover and in setting up of the second traffic redirection paths, considering the maximum allowed latency for a real-time application.

In Table 7.2, we summarize the results obtained from the extensive simulations of the developed mobility management solutions, and compare them in terms of performance metrics, functions and implementation parameters. In 


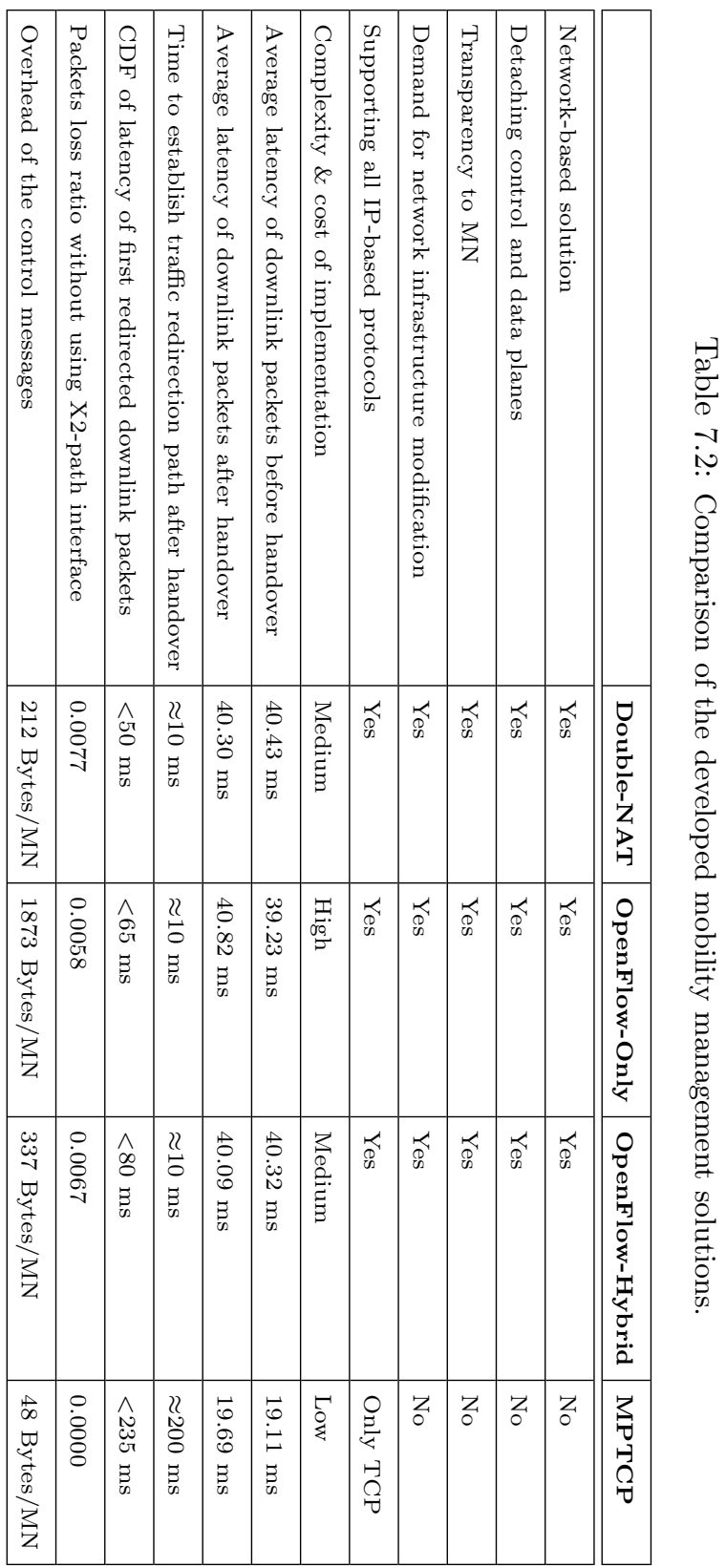


contrast to the NAT-based and SDN-based solutions, which both are networkbased solutions and hide any implementation functions from the end points, the MPTCP-based solution implements the MNs' mobility management functions in the transport layer of the end host entities, but keeps the network infrastructure intact. The NAT-based and SDN-based solutions demand to introduce a few new components in the network and some infrastructural modification, and accordingly provide more complexity on implementation, compared to the MPTCP-based solution. The experiments results show that, the NAT-based and SDN-based solutions outperforms the MPTCP-based solution on establishing the MNs traffic redirection paths after handover. However, MPTCP-based solution provides lower packets loss ratio and overhead of the control messages during MNs handover.

\subsection{Summary of Contributions}

In this section we list the main contributions of this thesis per chapter.

[Chapter 2]

- Proposed an approach for decentralization of the LTE network architecture.

- Detailed the essential modifications in the current EPC data and control planes, to be used for supporting MNs' mobility within the LTE network with decentralized architecture.

- Modified the current standard LTE's X2-based handover procedure with SGW relocation, to be used to handle IP traffic forwarding and session continuity for the MNs handed over between different S/PGWs.

- Extended the NS3-LENA simulation environment to implement and evaluate the functionality of the proposed approach. The source code for this implementation can be accessed via the link provided in appendix B.

[Chapter 3]

- Presented in detail the necessary background information about the GTP and PMIP protocols, the commonly used mobility management solutions in the current LTE, control and data planes for 3GPP access.

- Developed models of the network topology, MN's traffic and mobility, network link and router traffic and latency for the propose of study carried out in this chapter. 
- Conducted a hybrid study comprising simulation and analytical models to quantify, analysis and compare the performance and scalability of the current and decentralized LTE network architectures. The link of the source code used for this study can be found in appendix B.

[Chapter 4]

- Developed a NAT-based mobility management solution, that can seamlessly handle MN's handover procedure during a S/PGW relocation.

- Provided a detailed discussion on the functionalities of the control and data planes, messaging, architecture and components of the proposed solution, to realize its integration on the LTE network with decentralized architecture.

- Extended the NS3-LENA simulation environment to implement and evaluate the functionality and performance of the proposed approach. The link of the source code for implementation of this approach can be found in appendix B.

[Chapter 5]

- Developed two SDN-based mobility management solutions, that can seamlessly handle MN's mobility handed over between two S/PGWs. The solutions differ based on using the OpenFlow-only or OpenFlow-hybrid switches for implementation.

- Provided a detailed discussion on the functionalities of the control and data planes, messaging, architecture and components of the proposed solutions, to realize their integration on the decentralized LTE architecture.

- Extended the NS3-LENA simulation environment to implement and evaluate the functionality and performance of the proposed approaches. The source code to implement all of the modules for these approaches can be found via the link shown in appendix B.

[Chapter 6]

- Developed a MPTCP-based mobility management solution, that can provide seamless TCP traffic redirecting and session continuity for the MNs moving among S/PGWs. The developed solution handles the MNs' handover procedure without demanding an additional network interface on the MNs. 
- Provided a detailed discussion on the functionalities of the control and data planes, messaging and architecture of the proposed solution, to realize its integration on the decentralized LTE architecture.

- Extended the NS3-LENA simulation environment to implement and evaluate the functionality and performance of the proposed approach. The source code used to develop this approach is publicly available via the link presented in appendix B.

\subsection{Future Research}

In this section we set out research directions to extend the work presented in this thesis.

- In the current LTE system, besides the traffic forwarding and mobility anchoring functions, the central PGW is also in charge of other tasks and procedures such as policy enforcement, packet filtering, packet screening, lawful interception, and charging. In the decentralized LTE architecture as discussed in Chapter 2, the PGW and SGW functions are co-located into a single entity (i.e., S/PGW). This demands a common template of above mentioned functions for the distributed S/PGWs to carry out the unique regulations for the MNs handed over among them. For this propose, some additional modifications on the $3 \mathrm{GPP}$ standard procedures may need to be accomplished. Furthermore, some extra network connections and synchronization mechanisms (e.g., memory sharing) may also need to be applied between the EPC components (e.g., S/PGW, MME and PCRF). These procedures were out of the scope of our research and were left for future research.

- The study in Chapter 3 helped us to assess the efficiency and scalability of the LTE system with different network architectures. The outcomes of this research provide an initial insight on the impact of the growing number of users on the current and decentralized LTE architectures. As future study, our analysis can be further extended by taking into account other parameters e.g., the additional control plane overhead, needed to carry out the core network features (e.g., traffic monitoring and filtering, policy controlling, accounting), the required investment and the level of complexity for modification the network architectures. Ultimately, this can provide the mobile network operators a trustworthy intuition on decision making and policy development procedures to accommodate the network for coping with the future mobile data traffic. 
- In Chapter 4 and Chapter 5, we developed two solutions based on NAT mechanism and SDN technology to handle MNs' mobility in the LTE network with decentralized architecture. Both approaches, potentially enable efficient mobility management without intervention of a centralized mobility anchor point. The NAT and SDN controllers are able to handle individual IP traffic flow and redirect it to the MN's location. In a large network with millions of users, multiple NAT or SDN controllers may need to be present. Such cases may need further investigations to discover proper approaches for e.g., placement of the controller, synchronization of database on the controllers, and for optimizing control signaling. The use of multiple controllers apart from increasing the reliability of the system, may also have an impact on the time, needed to setup the NAT tables on the routers (or flow tables on the SDN switches). This impact is yet to be researched.

- Multiprotocol Label Switching (MPLS) is a scalable traffic forwarding technique in which routing is performed by swapping a label on the packets instead of traditional IP destination lookup. Nowadays, there is an increasing trend towards the use of MPLS in mobile networks to benefit from its capabilities on improvement e.g., QoS, traffic engineering, and reliability. In the scenario of employing the MPLS technology to backhaul data traffic over the access and aggregation networks, future work can be resided in how to adapt our network layer-based solutions to cope with the MNs' motility and traffic forwarding requirements.

- The MPTCP-based mobility management approach, presented in Chapter 6 , keeps the network infrastructure intact and implements the whole functionality for supporting MN's mobility in the transport layer of the end host entities. For this, we assumed that the MNs and CNs are MPTCPaware entities. To avoid the MPTCP's inherent deployment problem at the endpoints, one important future direction would be to investigate on a MPTCP proxy function to address the TCP $\rightleftharpoons$ MPTCP translation on top of the mobile devices and corresponding services. MPTCP proxy makes it possible to take the advantages of available multipath access without requiring MPTCP support in the all end devices and internet servers. 



\section{Detailed Calculation of The Performance Metrics}

In this appendix, we present the detailed derivation of Latency and Processing Cost for data packet delivery in the EPC network with centralized and decentralized architectures, as presented in $\S 3.5 .2$ and $\S$ 3.5.3.

\section{A.1 Average Latency of Data Packet Delivery for an MN}

The average latency of data packet delivery for mobile node $i\left(A L D P D_{i}\right.$ in Eq. 3.5) is derived using Eq. A.1.

$$
A L D P D_{i}=L A P_{i}+\frac{\sum_{h_{i}=1}^{N_{h_{i}}} L H P_{h_{i}}}{N_{h_{i}}}+\frac{\sum_{k_{i}=1}^{N_{k_{i}}} L D P D_{k_{i}}}{N_{k_{i}}}
$$

In following, we calculate $A L D P D_{i}$ items for the EPC network with centralized and decentralized architectures using GTP and PMIP protocols.

\section{A.1.1 The Centralized Architecture}

-) $L A P_{i(G T P)}=\left\{\left[\tau_{t_{\left(C . S . R e q+t_{c}\right)}}+\tau_{t_{\left(C . S . R e s+t_{c}\right)}}+\tau_{t_{\left(M . B . R e q+t_{c}\right)}}+\tau_{t_{\left(M . B . R e s+t_{c}\right)}}\right]_{\text {in SGW }}\right\}$

$+\left\{\sum_{l=1, j=1}^{H_{S G W} W P G W}\left[(\delta+d)_{\left(C . S . R e q+t_{c}\right)_{l}}+(\delta+d)_{\left(M . B . R e q+t_{c}\right)_{l}}+\tau_{r_{\left(C . S . R e q+t_{c}\right)_{j}}}+\tau_{\left.r_{(M . B . R e q}+t_{c}\right)_{j}}\right]\right\}$

$+\left\{\sum_{l=1, j=1}^{{ }_{P} P G W \rightarrow S G W}\left[(\delta+d)_{\left(C . S . R e s+t_{c}\right)_{l}}+(\delta+d)_{\left(M . B . R e s+t_{c}\right)_{l}}+\tau_{r_{\left(\text {C.S.Res }+t_{c}\right)_{j}}}+\tau_{\left.r_{(M . B . R e s}+t_{c}\right)_{j}}\right]\right\}$

$+\left\{\left[\tau_{t_{\left(\text {C.S.Req }+t_{c}\right)}}+\tau_{t_{\left(\text {C.S.Res }+t_{c}\right)}}+\tau_{\left.t_{(M . B . R e q}+t_{c}\right)}+\tau_{\left.t_{(M . B . R e s}+t_{c}\right)}\right]\right.$ in $\left.P G W\right\}$

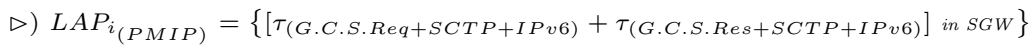




$$
\begin{aligned}
& +\left\{\left[\tau_{t_{(P . B . U+G R E)}}+\tau_{t_{(P . B . A+G R E)}}\right] \text { in } S G W\right\}
\end{aligned}
$$

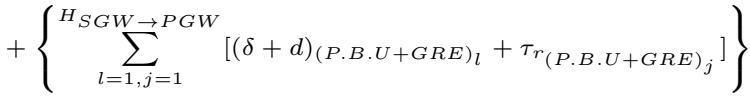

$$
\begin{aligned}
& +\left\{\sum_{l=1, j=1}^{{ }^{H} P G W \rightarrow S G W}\left[(\delta+d)_{(P . B . A+G R E)_{l}}+\tau_{\left.r_{(P . B . A+G R E)_{j}}\right]}\right]\right\} \\
& +\left\{\left[\tau_{t(P . B . U+G R E)}+\tau_{t(P . B . A+G R E)}\right] \text { in } P G W\right\} \\
& \text { •) } L H P_{h_{i(G T P)}}^{\dagger}=\left\{\left[\tau_{t_{\left(M . B . R e q+t_{c}\right)}}+\tau_{t_{\left(M . B . R e s+t_{c}\right)}}\right] \text { in } S G W\right\} \\
& +\left\{\sum_{l=1, j=1}^{{ }^{H_{S}}{ }^{P}{ }^{P G W}}\left[(\delta+d)_{\left(M . B . R e q+t_{C}\right)_{l}}+\tau_{r_{\left(M . B . R e q+t_{c}\right)_{j}}}\right]\right\} \\
& +\left\{\sum_{l=1, j=1}^{{ }^{H} P G W S G W}\left[(\delta+d)_{\left(M . B . R e s+t_{C}\right)_{l}}+\tau_{r_{\left(M . B . R e s+t_{C}\right)_{j}}}\right]\right\} \\
& +\left\{\left[\tau_{\left.t_{(M . B . R e q}+t_{C}\right)}+\tau_{\left.t_{(M . B . R e s}+t_{C}\right)}\right] \text { in PGW }\right\}
\end{aligned}
$$

$\left.{ }^{\dagger}\right)$ During every $S G W$ handover the $L H P_{h_{i(G T P)}}$ is repeated three times for eNodeB relocation and one time for $S G W$ relocation by replacing $S G W \rightarrow S G W^{\prime}$.

๖) $L H P_{h_{i(P M I P)}}=3 \times\left\{\left[\tau_{(\text {G.C.S.Req }+S C T P+I P v 6)}+\tau_{(\text {G.C.S.Res }+S C T P+I P v 6)}\right]\right.$ in SGW $\}$

$+\left\{\left[\tau_{(G . C . S . R e q+S C T P+I P v 6)}+\tau_{(\text {G.C.S.Res }+S C T P+I P v 6)}\right]\right.$ in $\left.S G W^{\prime}\right\}$

$+\left\{\left[\tau_{t(P . B . U+G R E)}+\tau_{t(P . B . A+G R E)}\right]\right.$ in $\left.S G W^{\prime}\right\}$

$+\left\{\sum_{l=1, j=1}^{{ }^{H} S G W^{\prime} \rightarrow P G W}\left[(\delta+d)_{(P . B . U+G R E)_{l}}+\tau_{r_{(P . B . U+G R E)_{j}}}\right]\right\}$

$+\left\{\sum_{l=1, j=1}^{{ }^{H} P G W \rightarrow S G W^{\prime}}\left[(\delta+d)_{(P . B . A+G R E)_{l}}+\tau_{r_{(P . B . A+G R E)_{j}}}\right]\right\}$

$+\left\{\left[\tau_{t}(P . B . U+G R E)+\tau_{t(P . B . A+G R E)}\right]\right.$ in $\left.P G W\right\}$

$$
\begin{aligned}
& \text { •) } L D P D_{k_{i(G T P)}}=\left\{\sum_{l=1, j=1}^{{ }^{H} C N \rightarrow P G W}\left[(\delta+d)_{(p)_{l}}+\tau_{r_{(p)}}\right]\right\}+\left\{\tau_{t_{\left(p+t_{u}\right)_{P G W}}}\right\}+\left\{\tau_{t}\left(p+t_{u}\right)_{S G W}\right\} \\
& +\left\{\sum_{l=1, j=1}^{H_{P G} W \rightarrow S G W}\left[(\delta+d)_{\left(p+t_{u}\right)_{l}}+\tau_{r_{\left(p+t_{u}\right)_{j}}}\right]\right\} \\
& +\left\{\tau_{t_{\left(p+t_{u}\right)_{S G W}}}+(\delta+d)_{\left(p+t_{u}\right)_{l}}+\tau_{r_{\left(p+t_{u}\right)_{j}}}+\tau_{\left(_{\left(p+t_{u}\right)_{S G W^{\prime}}}\right.}\right\}^{\dagger \dagger}
\end{aligned}
$$


${ }^{\dagger \dagger}$ ) This expression equals to zero if there is no a handover during sessiontime.

$\triangleright) L D P D_{k_{i(P M I P)}}$ is calculated the same as the $L D P D_{k_{i(G T P)}}$ by replacing $t_{u} \rightarrow$ GRE.

\section{A.1.2 The Decentralized Architecture}

-) $L A P_{i_{(G T P)}}=\left\{\left[\tau_{p_{(\text {C.S.Req })}}+\tau_{p_{(\text {C.S.Res })}}+\tau_{p_{(\text {M.B.Req })}}+\tau_{p_{(M . B . R e s)}}\right]\right.$ in S/PGW $\}$

D) $L A P_{(P M I P)}=\left\{\left[\tau_{p_{(G . C . S . R e q+S C T P+I P v 6)}}\right]\right.$ in $\left.S / P G W\right\}$

$+\left\{\left[\tau_{p_{(P . B . U)}}+\tau_{p_{(P . B . A)}}\right]\right.$ in $\left.S / P G W\right\}$

•) $L H P_{h_{i(G T P)}}=3 \times\left\{\left[\tau_{p_{(M . B . R e q)}}+\tau_{p_{(M . B . R e s)}}\right]\right.$ in $\left.S / P G W\right\}$

$+\left\{\left[\tau_{p_{(M . B . R e q)}}+\tau_{p_{(M . B . R e s)}}\right]\right.$ in $\left.S / P G W^{\prime}\right\}$

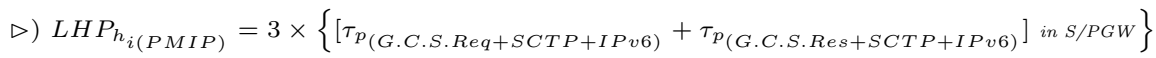

$+\left\{\left[\tau_{p_{(G . C . S . R e q+S C T P+I P v 6)}}+\tau_{p_{(G . C . S . R e s+S C T P+I P v 6)}}+\tau_{p_{(P . B . U)}}+\tau_{p_{(P . B . A)}}\right]\right.$ in $\left.S / P G W^{\prime}\right\}$

-) $L D P D_{k_{i(G T P)}}=\left\{\sum_{l=1, j=1}^{{ }^{H} C N \rightarrow S / P G W}\left[(\delta+d)_{(p)_{l}}+\tau_{r_{(p)}}\right]\right\}$

$+\left\{\tau_{\left(_{\left(p+t_{u}\right)_{S / P G W}}\right.}+(\delta+d)_{\left(p+t_{u}\right)_{l}}+\tau_{r_{\left(p+t_{u}\right)_{j}}}+\tau_{t_{\left(p+t_{u}\right)_{S / P G W^{\prime}}}}\right\}^{\dagger \dagger \dagger}$

${ }^{\dagger \dagger}$ ) This expression equals to zero if there is no a handover during session time.

$\triangleright) L D P D_{k_{i(P M I P)}}$ is calculated the same as the $L D P D_{k_{i(G T P)}}$ by replacing $t_{u} \rightarrow$ GRE.

\section{A.2 Average processing Cost of Data Packet Delivery for an MN}

The average processing cost of data packet delivery for a mobile node $i$ $\left(A C D P D_{i}\right.$ in Eq. 3.7) is calculated using Eq. A.2.

$$
A C D P D_{i}=C A P_{i}+\frac{\sum_{h_{i}=1}^{N_{h_{i}}} C H P_{h_{i}}}{N_{h_{i}}}+\frac{\sum_{k_{i}=1}^{N_{k_{i}}} C D P D_{k_{i}}}{N_{k_{i}}}
$$




\section{A.2. AVERAGE PROCESSING COST OF DATA PACKET}

DELIVERY FOR AN MN

This section presents calculation of $A C D P D_{i}$ items, in the EPC network with centralized and decentralized architectures for both GTP and PMIP protocols.

\section{A.2.1 The Centralized Architecture}

-) $\operatorname{CAP}_{i_{(G T P)}}=\left\{\left[C_{t_{\left(C . S . R e q+t_{c}\right)}}+C_{t_{\left(\text {C.S.Res }+t_{c}\right)}}+C_{t_{\left(M . B . R e q+t_{c}\right)}}+C_{t_{\left(M . B . R e s+t_{c}\right)}}\right]_{\text {in } S G W}\right\}$

$+\left\{\sum_{j=1}^{H_{S G} W \rightarrow{ }^{P G W}}\left[C_{\left.r_{(} \text {C.S.Req }+t_{c}\right)_{j}}+C_{r_{\left(M . B . R e q+t_{c}\right)_{j}}}\right]\right\}$

$+\left\{\sum_{j=1}^{H_{P G} W \rightarrow S G W}\left[C_{\left.r_{(} \text {C.S.Res }+t_{c}\right)_{j}}+C_{r_{\left(M . B . R e s+t_{c}\right)_{j}}}\right]\right\}$

$+\left\{\left[C_{t_{\left(C . S . R e q+t_{c}\right)}}+C_{t_{\left(C . S . R e s+t_{c}\right)}}+C_{t_{\left(M . B . R e q+t_{c}\right)}}+C_{t_{\left(M . B . R e s+t_{c}\right)}}\right]\right.$ in PGW $\}$

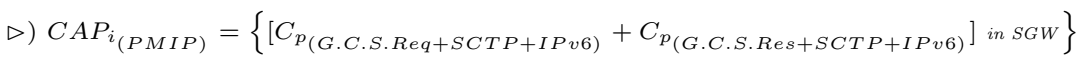

$+\left\{\left[C_{t_{(P . B . U+G R E)}}+C_{t_{(P . B . A+G R E)}}\right.\right.$ in $\left.S G W\right\}+\left\{\sum_{j=1}^{H_{S G W} \rightarrow P G W} C_{r_{(P . B . U+G R E)_{j}}}\right\}$

$+\left\{\sum_{j=1}^{H_{P G W} \rightarrow S G W} C_{r_{(P . B . A+G R E)_{j}}}\right\}+\left\{\left[C_{t_{(P . B . U+G R E)}}+C_{t_{(P . B . A+G R E)}}\right]_{\text {in } P G W}\right\}$

-) $C H P_{h_{i(G T P)}^{\ddagger}}^{\ddagger}=\left\{\left[C_{t_{\left(M . B . R e q+t_{c}\right)}}+C_{\left.t_{(M . B . R e s}+t_{c}\right)}\right]\right.$ in $\left.S G W\right\}+\left\{\sum_{j=1}^{H_{S G W} \rightarrow P G W} C_{\left.r_{\left(M . B . R e q+t_{c}\right.}\right)_{j}}\right\}$

$+\left\{\sum_{j=1}^{H_{P G W} S G W} C_{r_{\left(M . B . R e s+t_{c}\right)_{j}}}\right\}+\left\{\left[C_{t_{\left(M . B . R e q+t_{c}\right)}}+C_{\left.t_{\left(M . B . R e s+t_{c}\right)}\right] \text { in } P G W}\right\}\right.$

$\left.{ }^{\ddagger}\right)$ During every $S G W$ handover the $C H P_{h_{i(G T P)}}$ is repeated three times for eNodeB relocation and one time for $S G W$ relocation by replacing $S G W \rightarrow S G W^{\prime}$.

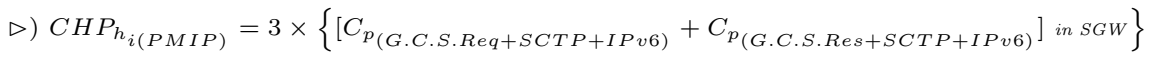

$+\left\{\left[C_{p_{(G . C . S . R e q+S C T P+I P v 6)}}+C_{p_{(G . C . S . R e s+S C T P+I P v 6)}}\right]\right.$ in $\left.S G W^{\prime}\right\}$

$+\left\{\left[C_{t_{(P . B . U+G R E)}}+C_{t_{(P . B . A+G R E)}}\right]\right.$ in $\left.S G W^{\prime}\right\}+\left\{\sum_{j=1}^{H_{S G W^{\prime} \rightarrow P G W}} C_{r_{(P . B . U+G R E)_{j}}}\right\}$

$+\left\{\sum_{j=1}^{{ }^{H} P G W \rightarrow S G W^{\prime}} C_{r_{(P . B . A+G R E)}}\right\}+\left\{\left[C_{t_{(P . B . U+G R E)}}+C_{t_{(P . B . A+G R E)}}\right]_{\text {in } P G W}\right\}$ 
•) $C D P D_{k_{i(G T P)}}=\left\{\sum_{j=1}^{{ }^{H} C{ }^{N \rightarrow P G W}} C_{r_{(p)_{j}}}\right\}+\left\{C_{t_{\left(p+t_{u}\right)_{P G W}}}\right\}+\left\{\sum_{j=1}^{{ }^{H} P G W \rightarrow S G W} C_{r_{\left(p+t_{u}\right)_{j}}}\right\}$ $+\left\{C_{t_{\left(p+t_{u}\right)_{S G W}}}\right\}+\left\{C_{t_{\left(p+t_{u}\right)_{S G W}}}+C_{r_{\left(p+t_{u}\right)_{j}}}+C_{t_{\left(p+t_{u}\right)_{S G W^{\prime}}}}\right\}^{\ddagger \ddagger}$

$\left.{ }^{\ddagger \ddagger}\right)$ This expression equals to zero if there is no a handover during sessiontime.

$\triangleright) C D P D_{k_{i(P M I P)}}$ is calculated the same as the $C D P D_{k_{i(G T P)}}$ by replacing the $t_{u} \rightarrow$ GRE.

\section{A.2.2 The Decentralized Architecture}

-) $C A P_{i_{(G T P)}}=\left\{\left[C_{p_{(\text {C.S.Req })}}+C_{p_{(\text {C.S.Res })}}+C_{p_{(M . B . R e q)}}+C_{p_{(M . B . R e s)}}\right]\right.$ in S/PGW $\}$

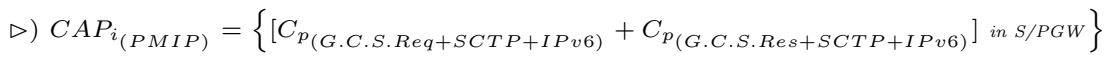
$+\left\{\left[C_{p_{(P . B . U)}}+C_{p_{(P . B . A)}}\right]\right.$ in $\left.S / P G W\right\}$

-) $C H P_{h_{i(G T P)}}=3 \times\left\{\left[C_{p_{(M . B . R e q)}}+C_{p_{(M . B . R e s)}}\right]\right.$ in $\left.S / P G W\right\}$

$+\left\{\left[C_{p_{(M . B . R e q)}}+C_{p_{(M . B . R e s)}}\right]\right.$ in $\left.S / P G W^{\prime}\right\}$

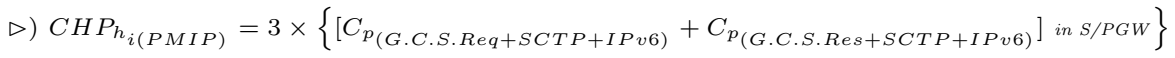
$+\left\{\left[C_{p_{(G . C . S . R e q+S C T P+I P v 6)}}+C_{p_{(G . C . S . R e s+S C T P+I P v 6)}}+C_{p_{(P . B . U)}}+C_{p_{(P . B . A)}}\right]\right.$ in S/PGW, $\}$

•) $C D P D_{k_{i(G T P)}}=\left\{\sum_{j=1}^{{ }^{H} C N \rightarrow S / P G W} C_{r_{(p)}}\right\}$ $+\left\{C_{\left.t_{(p+t u)}\right)_{S / P G W}}+C_{r_{(p+t u)_{j}}}+C_{t_{(p+t u)}}{ }_{S / P G W^{\prime}}\right\}^{\ddagger \ddagger \ddagger ~}$

‡¥) This expression equals to zero if there is no a handover during session time.

D) $C D P D_{k_{i(P M I P)}}$ is calculated the same as the $C D P D_{k_{i(G T P)}}$ by replacing the $t_{u} \rightarrow$ GRE. 



\section{Open Data Management}

In this appendix, we provide the links to access the source code used in this thesis. Table B.1 presents a brief description of the source code used per each chapter and lists the corresponding URLs to access.

Table B.1: Description and URLs of the source code used per each chapter.

\begin{tabular}{|l|l|l|}
\hline Chapter & Brief Description & Source Code \\
\hline Chapter 2 & $\begin{array}{l}\text { The extended NS3-LENA simulation environment to imple- } \\
\text { ment an LTE network with distributed S/PGW, as well as } \\
\text { the modified LTE's X2-based handover procedure to handle } \\
\text { MN's movement during a S/PGW relocation within the EPC } \\
\text { of an LTE network with decentralized architecture. }\end{array}$ & $\begin{array}{l}\text { MortezaKarimzadeh/ } \\
\text { DNAT_Mobility_LTE }\end{array}$ \\
\hline Chapter 3 & $\begin{array}{l}\text { The developed code in the MATLAB environment for imple- } \\
\text { mentation of all the components of our hybrid modeling study, } \\
\text { as well as for the detailed calculation of the performance met- } \\
\text { rics. }\end{array}$ & $\begin{array}{l}\text { MortezaKarimzadeh/ } \\
\text { Hybrid_Modeling_LTE }\end{array}$ \\
\hline Chapter 4 4 & $\begin{array}{l}\text { The extended NS3-LENA simulation environment to imple- } \\
\text { ment and evaluate the functionality and performance of the } \\
\text { NAT-based solution to handle MN's mobility above the EPC } \\
\text { of the LTE network with decentralized architecture. }\end{array}$ & $\begin{array}{l}\text { MortezaKarimzadeh/ } \\
\text { DNAT_Mobility_LTE }\end{array}$ \\
\hline Chapter 5 5 & $\begin{array}{l}\text { The extended NS3-LENA simulation environment to imple- } \\
\text { ment and evaluate the functionality and performance of the } \\
\text { SDN-based solutions to handle MN's mobility above the EPC } \\
\text { of the LTE network with decentralized architecture. }\end{array}$ & $\begin{array}{l}\text { MortezaKarimzadeh/ } \\
\text { SDN_Mobility_LTE }\end{array}$ \\
\hline Chapter 6 & $\begin{array}{l}\text { The extended NS3-LENA simulation environment to imple- } \\
\text { ment and evaluate the functionality and performance of the } \\
\text { MPTCP-based solution to handle MN's mobility above the } \\
\text { EPC of the LTE network with decentralized architecture. }\end{array}$ & $\begin{array}{l}\text { MortezaKarimzadeh/ } \\
\text { MPTCP_Mobility_LTE }\end{array}$ \\
\hline
\end{tabular}





\section{Bibliography}

[1] Host Identity Protocol. https://tools.ietf .org/html/rfc5201. 2008.

[2] ISO 8601 Data Elements and Interchange Formats. https://en.wikipedia. org/wiki/ISO_8601. 2017.

[3] LTE-EPC Network Simulator. http://networks.cttc.es/mobile-networks/ software-tools/lena/. 2015.

[4] MPTCP kernel on smartphones. https://multipath-tcp.org/pmwiki.php/ Users/Android. 2017.

[5] MPTCP protocol implementation on ns-3. https://github.com/dgomezunican/ multipath-ns3.13. 2013.

[6] Network Address and Port Translation (NAT) models. https://www.nsnam. org/wiki/GSOC2012Projects. 2012.

[7] OpenFlow Switch Specification Version 1.5.0. https://www.opennetworking. org/images/stories/downloads/sdn-resources/onf-specifications/ openflow/openflow-switch-v1.5.0.noipr.pdf. 2014.

[8] OpenFlow switch support. https://www.nsnam.org/docs/models/html/ openflow-switch.html. 2013.

[9] Quality of Service for Voice over IP. http://www.cisco.com/c/en/us/td/docs/ ios/solutions_docs/qos_solutions/QoSVoIP/QoSVoIP.pdf. 2011.

[10] Rocketfuel: An ISP Topology Mapping Engine. http://research.cs. washington.edu/networking/rocketfuel/. 2003.

[11] The Internet Traffic Statistics Archive. http://stats.simpleweb.org/ statistics.php. 2017.

[12] Use Multipath TCP to create backup connections for iOS. https://support. apple.com/en-us/HT201373. 2017.

[13] Dealing with Density: The Move to Small-Cell Architectures. RUCKUS Wireless White paper, 2014.

[14] Ericsson mobility report, November 2016. 
[15] Cisco Visual Networking Index, Global Mobile Data Traffic Forecast Update, 2016-2021., March 2017.

[16] 3GPP TS 23.829. Technical Specification Group Services and System Aspects; Local IP Access and Selected IP Traffic Offload (LIPA-SIPTO), Release 10. 2011.

[17] 3GPP SR 23.859. Technical Specification Group Services; LIPA Mobility and SIPTO at the Local Network, Release 11 and 12. 2012.

[18] 3GPP TS 36.300. LTE; Evolved Universal Terrestrial Radio Access (E-UTRA) and Evolved Universal Terrestrial Radio Access Network (E-UTRAN). 2016.

[19] 3GPP.TS.23.060. Digital cellular telecommunications system (Phase 2+); Universal Mobile Telecommunications System (UMTS); General Packet Radio Service (GPRS); Service description; Stage 2. 2011.

[20] 3GPP.TS.23.401. General Packet Radio Service (GPRS) Enhancements for Evolved Universal Terrestrial Radio Access Network (E-UTRAN) Access. 2011.

[21] 3GPP.TS.23.402. Universal Mobile Telecommunications System (UMTS); LTE. 2015.

[22] 3GPP.TS.29.274. 3GPP Evolved Packet System (EPS); Evolved General Packet Radio Service (GPRS) Tunnelling Protocol for Control plane (GTPv2-C); Stage 3. 2015 .

[23] 3GPP.TS.32.251. Technical Specification Group Services and System Aspects; Telecommunication management; Charging management; Packet Switched (PS) domain charging. 2015.

[24] M. Agiwal, A. Roy, and N. Saxena. Next Generation 5G Wireless Networks: A Comprehensive Surve.y. Journal of Communications Surveys and Tutorials, Vol 18, 2016.

[25] I. Ahmad, M. Liyanage, S. Namal, M. Ylianttila, A. Gurtov, M. Eckert, T. Bauschert, Z. Faigl, L. Bokor, and E. Saygun. New concepts for traffic, resource and mobility management in software-defined mobile networks. In Proceedings of the Wireless On-demand Network Systems and Services (WONS) Conference, 2016.

[26] I. F. Akyildiz, P. Wang, and S. Lin. SoftAir: A software defined networking architecture for 5G wireless systems. Journal of Computer Networks, Vol 85, 2015 .

[27] A. Alheid, A. Doufexi, and D. Kaleshi. A study on MPTCP for tolerating packet reordering and path heterogeneity in wireless networks. In Proceedings of the IFIP/IEEE Wireless Days (WD) Conference, 2016. 
[28] D. Ammar, T. Begin, and I.G. Lassous. A New Tool for Generating Realistic Internet Traffic in NS-3. In Proceedings of the 4th International ICST Conference on Simulation Tools and Techniques (SIMUTools), 2011.

[29] T. D. Assefa, R. Hoque, E. Tragos, and X. Dimitropoulos. SDN-based local mobility management with X2-interface in femtocell networks. In Proceedings of the Workshop IEEE Computer Aided Modeling and Design of Communication Links and Networks (CAMAD), 2017.

[30] P. Bertin, S. Bonjour, and J.M. Bonnin. Distributed or Centralized Mobility? In Proceedings of the IEEE Global Telecommunications Conference (GLOBECOM), 2009.

[31] Z. F. L. Bokor and S. Imre. Flat Architectures: Towards Scalable Future Internet Mobility. In Proceedings of the Future Internet, 2011.

[32] O. Bonaventure, C. Paasch, and G. Detal. Use Cases and Operational Experience with Multipath TCP. In Internet draft, MPTCP Working Group, 2016. https: //tools.ietf.org/html/draft-ietf-mptcp-experience-07.

[33] A. Bradaia, A. Benslimaneb, and K. D.Singhc. Dynamic Anchor Points Selection for Mobility Management in Software Defined Networks. Journal of Network and Computer Applications, Vol 57, 2015.

[34] H. Chan, P. Seite, K. Pentikousis, and A. Dutta. Distributed Mobility Management Framework. In Internet draft, DMM group, 2014.

[35] H. A. Chan, H. Yokota, J. Xie, P. Seite, and D. Liu. Distributed and Dynamic Mobility Management in Mobile Internet: Current Approaches and Issues. Journal of Communications, Vol 6, 2011.

[36] Y. Chen, E. M. Nahum, and R. J. Gibbens. Characterizing 4G and 3G networks: Supporting mobility with multi-path tcp. Technical report, 2012.

[37] A. Croitoru, D. Niculescu, and C. Raiciu. Towards Wifi Mobility without Fast Handover. In Proceedings of the 12th Symposium on Networked Systems Design and Implementation (NSDI), 2015.

[38] M. Dawood, W. Fuhrmann, and B.V. Ghita. Assay of Multipath TCP for Session Continuity in Distributed Mobility Management. In Proceedings of the 11th International Network Conference (INC), 2016.

[39] S. Eido, P. Mitharwal, A. Gravey, and C. Lohr. MPTCP Solution for Seamless Local SIPTO Mobility. In Proceedings of the IEEE High Performance Switching and Routing (HPSR) Conference, 2015.

[40] D. Farinacci, D. Meye, and D. Lewis. The Locator/ID Separation Protocol. In Internet Engineering Task Force (IETF), RFC 5533, 2013. https://tools. ietf.org/html/rfc6830. 
[41] A. Ford, C. Raiciu, M. Handley, S. Barre, and J. Iyengar. Architectural Guidelines for Multipath TCP Development. In Internet Engineering Task Force (IETF), RFC 6182, 2011. http://www.potaroo.net/ietf/idref/rfc6182/.

[42] A. Ford, C. Raiciu, M. Handley, and O. Bonaventure. TCP Extensions for Multipath Operation with Multiple Addresses. In Internet Engineering Task Force (IETF), RFC 6824, 2013. https://tools.ietf.org/html/rfc6824.

[43] S. Frei, W. Fuhrmann, A. Rinkel, and B. Ghita. Signalling Effort Evaluation of Mobility Protocols within Evolved Packet Core Network. In Proceedings of the International Network Conference (INC), 2010.

[44] Y. Ganjali and N. McKeown. Update on Buffer Sizing in Internet Routers. Journal of ACM SIGCOMM, Vol 36, 2006.

[45] F. Giust, C. J. Bernardos, and A. Oliva. Analytic Evaluation and Experimental Validation of a Network-Based IPv6 Distributed Mobility Management Solution. Journal of IEEE Transactions on Mobile Computing, Vol 13, 2014.

[46] F. Giust, L. Cominardi, and C.J. Bernardos. Distributed Mobility Management for future $5 \mathrm{G}$ networks: overview and analysis of existing approaches. Journal of IEEE Communications Magazine, Vol 53, 2015.

[47] D. Gomez, P. Garrido, C. Rabadan, R. Aguero, and L. Munoz. TCP Performance Enhancement over Wireless Mesh Networks by means of the Combination of Multi-RAT Devices and the MPTCP Protocol. Journal of Network Protocols and Algorithms (NPA), Vol 6, 2014.

[48] 5G PPP Architecture Working Group. View on 5G Architecture. In European Conference on Networks and Communications (EUCNC), 2016.

[49] S. Gundavelli, K. Chowdhury, V. Devarapalli, B. Patil, and K. Leung. Proxy Mobile IPv6. In Network Working Group, RFC 5213, 2008. https://tools . ietf .org/html/rfc5213.

[50] T. Guo, A. ul Quddus, N. Wang, and R. Tafazolli. Local Mobility Management for Networked Femtocells Based on X2 Traffic Forwarding. Journal of Transactions on Vehicular Technology, Vol 62, 2013.

[51] S. Habib, J. Qadir, A. Ali, D. Habib, M. Li, and A. Sathiaseelan. The Past, Present, and Future of Transport-Layer Multipath. Journal of Network and Computer Applications, Vol 75, 2016.

[52] W. Hahn. 3GPP Evolved Packet Core support for distributed mobility anchors: Control enhancements for GW relocation. In Proceedings of the International Conference on ITS Telecommunications (ITST), 2011.

[53] W. Hahn. Flat 3GPP Evolved Packet Core. In Proceedings of the International Symposium on Wireless Personal Multimedia Communications (WPMC), 2011. 
[54] G. Hampel, A. Rana, and T. Klein. Seamless TCP Mobility Using Lightweight MPTCP Proxy. In Proceedings of the 11th ACM international symposium on Mobility management and Wireless access (MobiWac), 2013.

[55] A. A. Hassan. Design and Analysis of Distributed Mobility Management in IPv6 Mobile Networks. PhD thesis, Télécom Bretagne, Université de Rennes, 2014.

[56] M. Hata, M. Soylu, S. Izumi1, T Abe1, and T. Suganuma1. A Design of SDN Based IP Mobility Management Considering Inter-Domain Handovers and Its Evaluation. Journal of Advances in Science, Technology and Engineering Systems (ASTES), Vol 2, 2017.

[57] S. P. Hoogendoorn. Vehicle-Type and Lane-Specific Free Speed Distributions on Motorways: A Novel Estimation Approach Using Censored Observations. Journal of Transportation Research Board, Vol 1934, 2006.

[58] M. Hoogesteger, R. de O. Schmidt, and A. Pras. ITSA: Internet Traffic Statistics Archive. In Proceedings of the IEEE/IFIP Network Operations and Management Symposium (NOMS), 2016.

[59] W. A. Imtiaz, M. Afaq, and M. A. K. Babar. mSCTP Based Decentralized Mobility Framework. Journal of Computing Research Repository (CoRR), Vol $2,2013$.

[60] R. Irmer. Next Generation Mobile Networks Radio Access Performance Evaluation Methodology. In NGMN Alliance White paper, 2008.

[61] M. M. Isah. An improved locator identifier split architecture (ILISA) to enhance mobility. PhD thesis, Lancaster University, 2017.

[62] S. Jeon, S. Figueiredo, and R.L. Aguiar. On the impacts of distributed and Dynamic Mobility Management strategy: A simulation study. In Proceedings of the IFIP/IEEE Wireless Days (WDs) Conference, 2013.

[63] S. Jeon, N. Kang, and R.L. Aguiar D. Corujo. Comprehensive Performance Evaluation of Distributed and Dynamic Mobility Routing Strategy. Journal of Computer Networks, Vol 79, 2015.

[64] D. Johnson, C. Perkins, and J. Arkko. Mobility Support for IPv6. In Network Working Group, RFC 3775, 2004. https://tools.ietf.org/html/rfc3775.

[65] V. P. Kafle, Y. Fukushima, and H. Harai. New mobility paradigm with ID/locator split in the future Internet. In Proceedings of the Consumer Communications and Networking Conference (CCNC), 2014.

[66] J. Kang and K. Okamura. An Identifier/Locator Split Architecture for Multihoming and Mobility Support. Journal of Computer Science and Network Security (IJCSNS), Vol 13, 2013. 
[67] M. Karimzadeh, A. Sperotto, and A. Pras. Software Defined Networking to Improve Mobility Management Performance. In Proceedings of the International Conference on Autonomous Infrastructure, Management, and Security (AIMS), 2014.

[68] M. Karimzadeh, L. Valtulina, and G. Karagiannis. Applying SDN/OpenFlow in Virtualized LTE to support Distributed Mobility Management (DMM). In Proceedings of the 4th International Conference on Cloud Computing and Services Science (CLOSER). SciTePress, 2014.

[69] M. Karimzadeh, L. Valtulina, A. Pras, J.L. van den Berg, R. de O. Schmidt, M. Liebsch, and T. Taleb. Double-NAT Based Mobility Management for Future LTE Networks. In Proceedings of the IEEE Wireless Communications and Networking Conference (WCNC), 2017.

[70] M. Karimzadeh, L. Valtulina, J.L. van den Berg, P.G. Ortiz A. Pras, and R. Sadre. MultiPath TCP to Support User's Mobility in Future LTE Network. In Proceedings of the IFIP/IEEE Wireless and Mobile Networking Conference (WMNC), 2017.

[71] M. Karimzadeh, L. Valtulina, J.L. van den Berg, A. Pras, M. Liebsch, and T. Taleb. Software Defined Networking to Support IP Address Mobility in Future LTE Network. In Proceedings of the IFIP/IEEE Wireless Days (WD) Conference, 2017.

[72] M. Karimzadeh, J.L. van den Berg, R. de O. Schmidt, and A. Pras. Quantitative Comparison of the Efficiency and Scalability of the Current and Future LTE Network Architectures. Journal of Wireless Communications and Mobile Computing (WCMC), Vol 2017, 2017.

[73] M. Karimzadeh, Z. Zhao, L. Hendriks, R. de O. Schmidt, S. la Fleur, J.L. van den Berg, A. Pras, and T. Braun. Mobility and Bandwidth Prediction as a Service in Virtualized LTE Systems. In Proceedings of the IEEE International Conference on Cloud Networking (CLOUDNET), 2015.

[74] Y. Kim, H. Ko, S. Pack, and J. H. Lee. Comparative study of centralized mobility management and distributed mobility management on ID/locator separation architectures. In Proceedings of the Workshop the IEEE Global Telecommunications Conference (GLOBECOM), 2012.

[75] M. Liebsch. Per-Host Locators for Distributed Mobility Management. In Internet draft, DMM Working Group, 2012. https://tools.ietf.org/id/draftliebsch-mext-dmm-nat-phl-01.txt.

[76] T. Mahmoodi and S. Seetharaman. On using a SDN-based control plane in $5 G$ mobile networks. Wireless World Research Forum (WWRF), 2014. 
[77] J. Moilanen and T. Kim. Deliverable D6.2 VNF/SDN/EPC: integration and system testing. In $5 G$ Communication with a Heterogeneous, Agile Mobile network in the Pyeongchang Winter Olympic CompetitioN, 2017.

[78] J. C. Murillo. From Centralized to Decentralized Mobility Management a Contribution to Future Mobile Networks. PhD thesis, Universidad de Extremadura, 2015.

[79] M. Murtadha, N. Noordin, B. Ali, and F. Hashim. Design and evaluation of distributed and dynamic mobility management approach based on PMIPv6 and MIH protocols. Journal of Wireless Networks, Vol 21, 2015.

[80] T. T. Nguyen, C. Bonnet, and J. Harri. SDN-based distributed mobility management for 5G networks. In Proceedings of the IEEE Wireless Communications and Networking Conference (WCNC), 2016.

[81] E. Nordmark and M. Bagnulo. Shim6: Level 3 Multihoming Shim Protocol for IPv6. In Network Working Group, RFC 5533, 2009. https://tools.ietf.org/ $\mathrm{html} / \mathrm{rfc5533.}$

[82] M. Olsson and C. Mulligan. EPC and $4 G$ Packet Networks: Driving the Mobile Broadband Revolution. Academic Press, 2012.

[83] C. Paasch, G. Detal, F. Duchene, C.Raiciu, and O. Bonaventure. Exploring Mobile/WiFi Handover with Multipath TCP. In Proceedings of the ACM SIGCOMM workshop on Cellular networks (CellNet), 2012.

[84] J. Postel. Transmission Control Protocol. In Internet Engineering Task Force (IETF), RFC 793, 1981. https://tools.ietf.org/html/rfc793.

[85] A. Roozbeh. Distributed Cloud and De-centralized Control Plane: A Proposal for Scalable Control Plane for 5G. In Proceedings of the IEEE/ACM International Conference on Utility and Cloud Computing (UCC), 2015.

[86] R. R. Roy. Handbook of Mobile Ad Hoc Networks for Mobility Models. Springer, 2010 .

[87] M. I. Sanchez and A. l. Oliva V. Mancuso. Experimental evaluation of an sdnbased distributed mobility management solution. In Proceedings of the Workshop on Mobility in the Evolving Internet Architecture (MobiArch), 2016.

[88] C. Schindelhauer. Mobility in wireless networks. In Proceedings of the International Conference on Current Trends in Theory and Practice of Computer Science, 2006.

[89] C. Singhal and S. De. Resource allocation in next-generation broadband wireless access networks. IGI Global, 2017. 
[90] N. Spring, R. Mahajan, and D. Wetherall. Measuring ISP Topologies with Rocketfuel. Journal of ACM SIGCOMM Computer Communication Review, Vol 12, 2002 .

[91] K. Sun and Y. Kim. Flow Mobility Management in PMIPv6-based DMM (Distributed Mobility Management) Networks. Journal of Wireless Mobile Networks, Ubiquitous Computing, and Dependable Applications (JoWUA), Vol 5, 2014.

[92] Y. Sun, Y. Cui, W. Wang, T. Ma, Y. Ismailov, and X. Zheng. Mobility support in multi-path TCP. In Proceedings of the IEEE Communication Software and Networks (ICCSN), 2011.

[93] T. Taleb, K. Samdanis, and F. Filali. Towards supporting highly mobile nodes in decentralized mobile operator networks. In Proceedings of the IEEE International Conference on Communications (ICC), 2012.

[94] R. Trivisonno, R. Guerzoni, I. Vaishnavi, and D. Soldani. SDN-based 5G mobile networks: architecture, functions, procedures and backward compatibility. Journal of Emerging Telecommunications Technologies, Vol 26, 2015.

[95] L. Valtulina, M. Karimzadeh, G. Karagiannis, G. Heijenk, and A.Pras. Performance evaluation of a SDN/OpenFlow-based Distributed Mobility Management (DMM) approach in virtualized LTE systems. In Proceedings of the IEEE Global Communications Conference (GLOBECOM), 2014.

[96] B. Vejlgaard. Deployment Strategies for Heterogeneous Networks. Technical report, 2014.

[97] S. Vohra. An SDN Based Approach to a Distributed LTE-EPC. Master's thesis, Indian Institute of Technology Bombay, 2016.

[98] D. Wang, L. Zhang, Y. Qi, and A. U. Quddus. Localized Mobility Management for SDN-Integrated LTE Backhaul Networks. In Proceedings of the IEEE Vehicular Technology Conference (VTC), 2015.

[99] M. Wang, M. Georgiades, and R. Tafazolli. Signalling Cost Evaluation of Mobility Management Schemes for Different Core Network Architectural Arrangements in 3GPP LTE/SAE. In Proceedings of the IEEE Vehicular Technology Conference (VTC), 2008.

[100] Y. Wang and J. Bi. A solution for IP mobility support in software defined networks. In Proceedings of the IEEE International Conference on Computer Communication and Networks (ICCCN), 2014.

[101] J. Wilke. 5G Network Architecture and FMC. Technical report, 2017.

[102] W. Xiang, K. Zheng, and X. Sherman. 5G Mobile Communications. Springer, 2017. 
[103] L. Yi, H. Zhou, F. Ren, and H. Zhang. Analysis of Route Optimization Mechanism for Distributed Mobility Management. Journal of Networks, Vol 7, 2012.

[104] N. Zhang. Cost Efficiency of SDN in LTE-based Mobile Networks: Case Finland. In Proceedings of the International Workshop at Networked Systems (NetSys), 2015. 


\section{Acronyms}

ACDPD Average Processing Cost of Data Packet Delivery.

ACHP Average Processing Cost for Handover Procedure.

ALAP Average Latency of initial Attachment Procedure.

ALDPD Average Latency of Data Packet Delivery.

ALHP Average Latency for Handover Procedure.

CAP Processing Cost of initial Attachment Procedure.

CDF Cumulative Distribution Function.

CML Control Message Load.

CN Corresponding service Node.

D-PMIPv6 PMIPv6-based Distributed mobility management model.

DDMM Distributed and Dynamic Mobility Management.

DMA Dynamic Mobility Anchoring.

DMM Distributed Mobility Management.

DNR domain name registry.

DSMIP Dual Stack MIP.

E-UTRAN Evolved Universal Terrestrial RAN.

EIDs Endpoint Identifiers.

eNodeBs evolved NodeBs.

EPC Evolved Packet Core.

ePDG Evolved Packet Data Gateway.

EPS Evolved Packet System.

F-DMM Fully Distributed Mobility Management.

GR Growth Rate.

GRE Generic Routing Encapsulation.

GTP GPRS Tunneling Protocol.

HGW home gateway.

HIMALIS Heterogeneity Inclusion and Mobility Adaptation through ID/Locator Separation.

HIP Host Identity Protocol.

HNR host name registry. 
ILISA Locator/Identifier Split Architecture.

ILSMM Identifier/Locator Split architecture for Multi-homing and Mobility.

IP-CAN IP Connectivity Access Network.

IS

Increasing Slope.

LBI Linked EPS Bearer ID.

LIPA Local IP Access.

LISP Locator/ID Separation Protoco.

LNL Load of the Network Links.

LR Load of the network Routers.

LTE Long-Term Evolution.

MIP Mobile IP.

MME Mobility Management Entity.

MNs' Mobile Nodes'.

MPLS Multiprotocol Label Switching.

MPTCP Multipath-TCP.

multi-RAT multiple Radio Access Technology.

NAT Network Address Translation.

NFV Network Function Virtualization.

NV Network Virtualization.

OFSID OpenFlow Software Implementation Distribution.

P-DMM Partially Distributed Mobility Management.

PCRF Policy and Charging Rule Function.

PDN Packet Data Network.

PGW Packet Data Network Gateway.

PMIP Proxy Mobile IP.

PPBP Poisson Pareto Burst Process.

QoS quality of service.

R Ratio.

RAN Radio Access Network.

RLOCs Routing Locators.

SCTP Stream Control Transmission Protocol.

SDN Software Defined Network.

SGW Serving Gateway.

SGWs Serving Gateways.

Shim6 Multihoming Shim Protocol for IPv6.

SIPTO Selected IP Traffic Offload. 
TEID Tunnel Endpoint Identifier.

TFT Traffic Flow Template. 



\section{List of Publications}

Below is a list of papers that Morteza Karimzadeh published during the time he was a Ph.D. candidate at DACS, sorted in reverse chronological order:

1. M. Karimzadeh, J.L. van den Berg, R. de O. Schmidt, and A. Pras, Quantitative Comparison of the Efficiency and Scalability of the Current and Future LTE Network Architectures, Journal of Wireless Communications and Mobile Computing (WCMC), 2017.

2. M. Gholibeigi, N. Sarrionandia, M. Karimzadeh, M. Baratchi, J.L. van den Berg, and G. Heijenk, Reliable Vehicular Broadcast using 5G Device-to-Device Communication, In Proceedings of the IFIP/IEEE Wireless and Mobile Networking Conference (WMNC), 2017.

3. M. Karimzadeh, L. Valtulina, J.L. van den Berg, A. Pras, P. G. Ortiz, and R. Sadre, MultiPath TCP to Support User's Mobility in Future LTE Network, In Proceedings of the IFIP/IEEE Wireless and Mobile Networking Conference (WMNC), 2017.

4. Z. Zhao, M. Karimzadeh, T. Braun, A. Pras, and J.L. van den Berg, Cloudified Mobility and Bandwidth Prediction in Virtualized LTE Networks, In Proceedings of the IFIP/IEEE International Symposium on Integrated Network Management (IM), 2017.

5. M. Karimzadeh, L. Valtulina, J.L. van den Berg, A. Pras, M. Liebsch, and T. Taleb, Software Defined Networking to Support IP Address Mobility in Future LTE Network, In Proceedings of the IFIP/IEEE Wireless Days (WD) Conference, 2017.

6. M. Karimzadeh, L. Valtulina, A. Pras, J.L. van den Berg, R. de O. Schmidt, M. Liebsch, and T. Taleb, Double-NAT Based Mobility Management for Future LTE Networks, In Proceedings of the IEEE Wireless Communications and Networking Conference (WCNC), 2017.

7. M. B. Sousa, Z. Zhao, M. Karimzadeh, D. Palma, V. Fonseca, P. Simoes, T. Braun, J.L. van den Berg, A. Pras, and L. Cordeiro, Enabling a Mobility Prediction-aware Follow-Me Cloud Model, In Proceedings of the IEEE Local Computer Networks (LCN), 2016.

8. M. Karimzadeh, Z. Zhao, L. Hendriks, R. de O. Schmidt, S. la Fleur, J.L. van den Berg, A. Pras, T. Braun, and M. Corici, Mobility and Bandwidth Prediction as a Service in Virtualized LTE Systems, In Proceedings of the IEEE International Conference on Cloud Networking (CLOUDNET), 2015. 
9. Z. Zhao, M. Karimzadeh, T. Braun, A. Pras, and J.L. van den Berg, A Demonstration of Mobility Prediction as a Service in Cloudified LTE Networks, In Proceedings of the IEEE International Conference on Cloud Networking (CLOUDNET), 2015.

10. T. Taleb, M. Iulian Corici, C. Parada, A. Jamakovic, S. Ruffino, G. Karagiannis, M. Karimzadeh, and T. Magedanz, Virtualizing the LTE Evolved Packet Core $(E P C)$, In Proceedings of the IEEE European Conference on Networks and Communications (EUCNC), 2014.

11. M. Karimzadeh, L. Valtulina, and G. Karagiannis, Applying SDN/OpenFlow in Virtualized LTE to support Distributed Mobility Management (DMM), In Proceedings of the 4th International Conference on Cloud Computing and Services Science (CLOSER), 2014.

12. M. Karimzadeh, T. Satria, and G. Karagiannis, Utilizing ICN/CCN for service and VM migration support in virtualized LTE systems, In Proceedings of the 4th International Conference on Cloud Computing and Services Science (CLOSER), 2014.

13. M. Karimzadeh, A. Sperotto, and A. Pras, Software Defined Networking to Improve Mobility Management Performance, In Proceedings of the International Conference on Autonomous Infrastructure, Management, and Security (AIMS), 2014.

14. L. Ferreira, D. Pichon, A. Hatefi, A. Gomes, D. Dimitrova, T. Braun, G. Karagiannis, M. Karimzadeh, M. Branco, and L.M. Correia, An Architecture to offer Cloud-Based Radio Access Network as a Service, In Proceedings of the IEEE European Conference on Networks and Communications (EUCNC), 2014.

15. G. Karagiannis, A. Jamakovi, K. Briggs, M. Karimzadeh, C. Parada, M. J. Corici, T. Taleb, A. Edmonds, and T. M. Bohnert, Mobility and Bandwidth prediction in virtualized LTE systems: Architecture and Challenges, In Proceedings of the IEEE European Conference on Networks and Communications (EUCNC), 2014 .

16. L. Valtulina, M. Karimzadeh, G. Karagiannis, G. Heijenk, and A. Pras, Performance evaluation of a SDN/OpenFlow-based Distributed Mobility Management (DMM) approach in virtualized LTE systems, In Proceedings of the IEEE Global Communications Conference (GLOBECOM), 2014.

17. T. A. Satria, M. Karimzadeh, and G. Karagiannis, Performance evaluation of $I C N / C C N$ based service migration approach in virtualized LTE systems, In Proceedings of the IEEE International Conference on Cloud Networking (CLOUDNET), 2014.

18. A. Gomes, T. Braun, G. Karagiannis, M. Karimzadeh, M. Liebsch, A. Rodríguez, and P. Comi, Follow Me Cloud and Virtualization of (Multimedia) Services and Applications: Challenges and Possible Solutions, Workshop on Future Networks \& Mobile Summit 2013 (FUNEMS), 2013. 
19. G. Karagiannis, M. Karimzadeh, T. Taleb, T. Braun, and T. M. Bohnert, Applying Follow Me and Service Migration $\mathcal{E}$ Continuity Features in Cloud-based LTE Systems, Workshop on Future Networks \& Mobile Summit 2013 (FUNEMS), 2013.

20. I. Drago, R. de O. Schmidt, R. Hofstede, A. Sperotto, M. Karimzadeh, B.R.H.M. Haverkort, and A. Pras, Networking for the Cloud: Challenges and Trends, Journal of Praxis der Informationsverarbeitung und Kommunikation (PIK), 2013. 


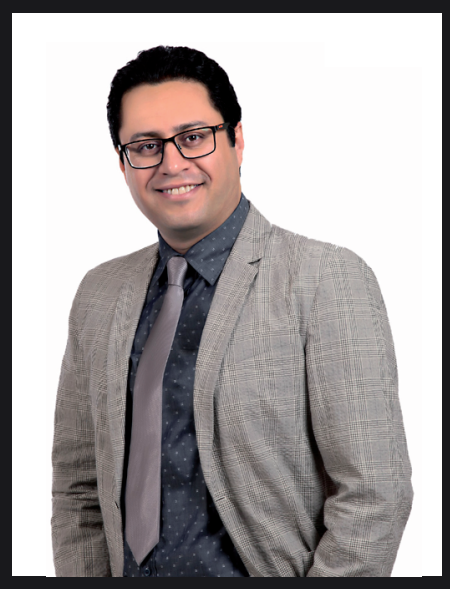

Morteza Karimzadeh received his Bachelor of Science (B.Sc.) degree in Electronics and Electrical Engineering in 2000 from the Urmia University, Iran, and his Master of Science (M.Sc.) degree in Communication Networks and Protocols Engineering in 2011 from the Tampere University of Technology, Finland. Prior to beginning his Ph.D. study, he worked as a researcher with Prof. Antonio Capone in Advanced Network Technologies Laboratory (ANTLab) at the Polytechnic University of Milan, since 2011 to 2013. In the period from March 2013 to August 2017, he was a Ph.D. candidate at the Design and Analysis of Communication Systems (DACS) group of the University of Twente, the Netherlands, under the supervision of Prof. Hans van den Berg and Prof. Aiko Pras. During this period he had the opportunity to take part in EU FP7 Mobile Cloud Networking (MCN) project.

ISSN 2589-4730

ISBN 978-90-365-4532-7

DOI 10.3990/1.9789036545327

https://doi.org/10.3990/1.9789036545327

Copyright @ 2018 Morteza Karimzadeh Motallebi Azar 